\title{
The sinus node and atrial fibrillation
}

Citation for published version (APA):

Kirchhof, C. J. H. J. (1989). The sinus node and atrial fibrillation. [Doctoral Thesis, Maastricht University]. Rijksuniversiteit Limburg. https://doi.org/10.26481/dis.19890928ck

Document status and date:

Published: 01/01/1989

DOI:

10.26481/dis.19890928ck

Document Version:

Publisher's PDF, also known as Version of record

\section{Please check the document version of this publication:}

- A submitted manuscript is the version of the article upon submission and before peer-review. There can be important differences between the submitted version and the official published version of record.

People interested in the research are advised to contact the author for the final version of the publication, or visit the DOI to the publisher's website.

- The final author version and the galley proof are versions of the publication after peer review.

- The final published version features the final layout of the paper including the volume, issue and page numbers.

Link to publication

\footnotetext{
General rights rights.

- You may freely distribute the URL identifying the publication in the public portal. please follow below link for the End User Agreement:

www.umlib.nl/taverne-license

Take down policy

If you believe that this document breaches copyright please contact us at:

repository@maastrichtuniversity.nl

providing details and we will investigate your claim.
}

Copyright and moral rights for the publications made accessible in the public portal are retained by the authors and/or other copyright owners and it is a condition of accessing publications that users recognise and abide by the legal requirements associated with these

- Users may download and print one copy of any publication from the public portal for the purpose of private study or research.

- You may not further distribute the material or use it for any profit-making activity or commercial gain

If the publication is distributed under the terms of Article $25 \mathrm{fa}$ of the Dutch Copyright Act, indicated by the "Taverne" license above, 
The Sinus Node and

Atrial Fibrillation 



\title{
The Sinus Node and Atrial Fibrillation
}

\author{
Proefschrift
}

ter verkrijging van de graad van doctor

aan de Rijksuniversiteit Limburg te Maastricht, op gezag van de Rector Magnificus, Prof. Dr. F.I.M. Bonke, volgens het besluit van het College van Dekanen, in het openbaar te verdedigen op

donderdag, 28 septémber 1989 om 13.30 uur

door

Carolus Jean Henri Jacques Kirchhof geboren 14 maart 1963 te Heerlen 


\section{Promotores:}

Prof. Dr. M.A. Allessie

Prof. Dr. F.I.M. Bonke

\section{Beoordelingscommissie:}

Prof. Dr. H.A.J. Struyker Boudier (vaarzitter), Rijksuniversiteit Limburg

Prof. Dr. L.N. Bouman, Universiteit van Amsterdam

Prof. Dr. A. Hasman, Rijksuniversiteit Limburg

Prof. Dr. J. Jalife, State University of New York

Prof. Dr. H.J.J. Wellens, Rijksuniversiteit Limburg

CIP-data Kaninklijke Bibliotheek "Den Haag

Klichhof, Carolus Jean Henri Jacques

The Sinus Node and Atrial Fibrillation / Carolus Jean Henrl Jacques Kirchhof - Maastricht: Datawyse - Ifl

Thesis Maastrlcht - With ref.. - With summary in Dutch ISBN $90-5291-003-0$

SISO 605.12 UDC 616.12:612(043.3)

Sub|ect heading: cardlology / physiology.

Financial support by the Netherland Heart Foundation, Organon International and Knoll b.v. for the publication of this thesis is gratefully acknowledged.

Uitgave: Datawyse Maastricht

Druk: Krips Repro Meppel 
"The history of the recognition of atrial fibrillation of the auricles will impress you with the dimness of our eyes and the opacity of the obstacles which embarrass our vision. You will know how blind we have been to things which, once seen, are so apparent:"

Sir Thomas Lewis, 1912

voor mijn Ouders aan Juliëtte 


\section{Table of Contents}

Preface

Chapter I: General Introduction

Chapter II: The influence of the atrial myocardium on impulse formation in the rabbit sinus node

(Pflogers Arch 410:198-203, 1987)

Chapter III: An easy and direct approach to investigate conduction properties of the rabbit sinus node

(N Cardlovasc Pharmacol 17:667-675, 1988)

Chapter IV: Effects of bepridil on conduction properties of the isolated rabbit sinus node and atrial myocardium

(J Cardiovasc Pharmacol 13:137-137, 1989)

Chapter V: Effects of verapamil, diltiazem and disopyramide on sinus function: a comparison with bepridil

(Eur J Pharmacol 160:369-376, 1989)

Chapter VI: Extracellular mapping of the sinus node region during atrial fibrillation

Chapter VII: Intracellular recordings from the sinus node during atrial fibrillation

Chapter VIII: Resumption of sinus rhythm after termination of atrial fibrillation

Chapter IX: General discussion

List of References

Summary

Samenvatting

Curriculum Vitae 


\section{PREFACE}

The present thesis describes the results of a series of experimental studies performed between 1983 and 1989 at the department of Physiology (chairman: Prof. Dr. R.S. Reneman) of the University of Limburg in Maastricht, The Netherlands. The general objective of these studies was to increase our insight into the functional characteristics of the mammalian sinus node and the relevance of this structure in the genesis and perpetuation of cardiac arrhythmias, particularly atrial fibrillation. Several clinical arrhythmias are known to be based on impaired sinus node function. In these cases the pathophysiological mechanism must be recognized in order to select the most appropriate therapy.

On behalf of the present studies young rabbits were sacrificed. The hearts of these animals were isolated and kept alive for several hours. The rabbit was the experimental animal of choice because in this species the sinus node is not completely embedded in the atrial myocardium as is the case in larger animals and in man. This enabled us to study the sinus node by means of direct electrical recording techniques. Several aspects of impulse formation and conduction in the sinus node were investigated, both under normal conditions, after administration of drugs, and during induced atrial fibrillation. After a general introduction (chapter l) in the electrophysiology of the sinus node and related cardiac arrhythmias, the results of the present studies are described in chapters II to VIII, followed by a general discussion in chapter IX. The contents of chapters II, III, IV, and V were recently published as individual papers in international journals. 
Chapter I

| GENERAL INTRODUCTION 


\section{Historical Overview}

The question 'What causes the mammalian heart to beat?' has been the driving force of philosophers and scientists for many centuries. Hippocrates ( $460-377$ B.C.) was one of the first who was interested in this prablem as written in his Corpus Hippocraticum [100]:

"Within the heart, there burns a sort of vestal flre, so subtle that it escapes immediately through any incision made to reveal it. Alr coming into the heart from the trachea and lungs falls upon the flame and is set into a violent effervescence which drives it out through the opening of the great arteries and at the same time causes the swelling of the heart known as the diastole."

In the early $16^{\text {th }}$ century this theory was modified by Sylvius according to the views of the current alchemists [100]:

"The alkaline venous blood, meeting the acid chyle and also the acid' bile, becomes effervescent and causes diastore."

In the second half of the $17^{\text {th }}$ century the purely chemical theories on the origination of the heartbeat were doubted. In anatomical studies nerves were identified which innervated the heart muscle. Based on these findings Willis introduced in 1664 the 'neurogenic' theory [100]. In analogy to other neuro-muscular systems, he proposed that these cardiac nerves provided the heart muscle with the necessant energy for its rhythmic contraction. This neurogenic theory dominated into the second half of the $18^{\text {th }}$ century, until Von Haller introduced in 1754 a completely different hypothesis on the origin of the heartbeat [278]:

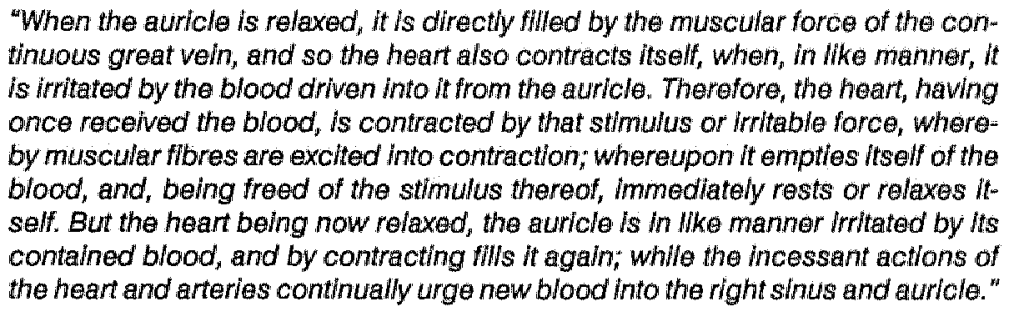

In the beginning Von Haller's relatively simple theory of an interplay between the inherent excitability of the heart muscle and the repetitive filling of the auricles with blood was strengthened by the finding that the rhythmic contractions of the heart maintained after cutting the extrinsic nerve supply. However, in the $19^{\text {th }}$ century Von 
Haller's theory was contradicted by the discovery of ganglionic cells in the heart muscle. The relevance of these findings was stressed by Gaskell in 1884 [82]:

"The discovery of the cardiac gangilia and especially their situation in that part of the heart - the sinus - from which it had been long recognized that the rhythmical beat took its origin, led instartly to the hypothesis that they played the chief part in causation of the beat, although at the same time they were recognized as appendages to the nerves and not separated independent structures."

Nevertheless, a neurogenic origin of the heartbeat became less plausible because of increasing evidence for the existence of intrinsic cardiac automaticity. In these days it was Engelmann who started to investigate this mechanism profoundly. He used the complicated suspension-technique in combination with a pantokymograph to register the contraction curves of different parts of the turtle heart. He observed that a mechanically induced extrasystole was followed by a compensatory pause in the rhythm of the heart. Analysis of these pauses led Engelmann to the conclusion that the heartbeat was initiated by the automatic generation of an impulse, independent of any neural activity. He reported his findings during a physiological meeting in 1896 [72]:

"Aan de ostia venosa wordt dus aanhoudend, niet periodiek, niet isorhytmisch met de systolen der ostia, irritatio-oorzaak geproduceerd. De organen waarin die automatische prikkels zich ontwikkelen, kunnen niet gangliencellen zijn. In vele stukjes der holle aderen ('w. cavae superiores vooral), die na het uitsnijden nog uren lang regelmatig hadden geklopt, heeft spreker bil het meest nauwgezet on derzoek geen gangliencellen kunnen vinden, slechts splervezelen: netsgewils vereenigde bundels van langwerpige dwarsgestreepte vezelcellen van den typus der hartsplercellen."

"Bll de geringe dimensies, wooral de zeer geringe dikte der wanden van de genoemde holle aderen, die een onderzoek in toto met sterke vergrootingen zeer gemakkelijk maakt, heeft het nlet vinden van gangllencellen de waarde wan een positief bew/s voor de afwezigheid van zenuwcentra. Zil zouden niet kunnen worden voorbllgezlem als ze er waren. Spreker komt dus tot de conclusie, dat de oorzaak der normale bowegingen moet gezocht worden in een automatische prikkel. baarheid der splervezelen; de automatisch opgewekte contractie plant zich dan door spiergeleiding, door het hele orgaan voort. Noch woor het ontstaan, noch voor de voortgelelding der systolische golf is dus de medewerking van zenuwen een vereischte: de normale hartsbeweging is in haar geheel van zuiver myogenen, niet van neurogenen aard."

Engelmann's results were in agreement with the earlier thoughts of Gaskell, and strengthened by the findings of other investigators like McWillam [175]. Thus, at the beginning of the $20^{\text {th }}$ century the 'myogenic theory' had become the dominant concept with respect to the origin of the heartbeat. 
Until these days the majority of studies on cardiac rhythmicity were dealing with the hearts of lower vertebrates. The pacemaker in the mammalian heart on the other hand, was not so easily identified. Many methods, either crude or quite sophisticated, were applied to the hearts of various species. One of the first valid studies in this context was performed by McWilliam in 1888 [175]. In cat hearts he found a thermosensitive spot at the terminal portion of the superior caval vein. Warming of this spot caused cardiac acceleration, whereas cooling it reduced the beating rate of the heart. Two decades later, in 1906, Adam localized this thermosensitive area more accurately in the rabbit and cat heart [1]. At the same time Langendorff and Lehmann described that excision of this region caused permanent cessation of atrial activity [151].

In 1907 Arthur Keith and his young assistant Martin Flack obtained the first substantial evidence for the existence of an automatic center in the right atrium [133]. In an extensive study, including various lower and higher vertebrates, these investigators invariably found a group of primitive fibers at the sino-auricular junction. They believed that: "....it is in them that the dominating rhythm of the heart normally begins". Because of these observations Keith and Flack are generally given credit for the discovery of the sinus node. In the same year Erlanger and Blackman repeated the experiments of Langendorff and Lehmann in dog hearts [73]. Surgical isolation of different parts of the right atrial muscle at the sino-auricular junction revealed that "...the region of the right auricle in the vicinity of the mouths of the great veins is possessed of the highest degree of rhythmicity".

In the following years Flack studied the influences of local hypothermia, electrical stimulation, clamping, pinching and ligation of the sinus node area, and determined the effects of atropine and muscarine on cardiac rhythm. His observations led him to the following conclusions in 1911 [78]:

"(1) At the sino-auricular node alone can the normal dominating rhythm of the beat be affected" a) by the application of cold', b) by electrical sthriflation and c) by mechanical stimulation such as pinching.

(2) At this spot the vagus and sympathetle nerves, particularly the right norves, exert their main effect upon the heart rhythm, appllcation of atropline and cold in most cases abolishes this effect, as does tight clamping of the nodes.

(3) Muscarine applied to the node slows the whole heart rhythm. It also first increases the effect of vagus stimulation and then abolishes it. Application of atropine to the node counteracts the effects of muscarine applied directly to the node or injected into the blood."

Both in physiology and clinical cardiology an important step foreward was attained by the possibilty to record the electrical activity of the heart. Already in 1856 , Kölliker and Müller demonstrated a cardiac action current by bringing a frog's 
nerve-muscle preparation in direct contact with a beating heart [143]. During these experiments two distinct electrical discharges were observed with every heartbeat. These early electrocardiographic attempts were followed by several studies in which the earliest types of the galvanometer and capillary electrometer were used $[71,223,224]$. In 1887 Wailer demonstrated the technique to register the human heartbeat electrically [280]. However, it was not until 1897 when the first satisfactory mammalian electrograms were recorded by Bayliss and Starling [16]. Important technical improvements in this field were made by the introduction of the string galvanometer by Einthoven in 1903 [70]. By means of this apparatus the electrical activity preceding each heartbeat could be examined with such increased precision that the other forms of registration were rapidly superseded.

Sir Thomas Lewis was the first who used the string-galvanometer to study the electrical origin of the heartbeat in dogs. He found that faradic stimulation of the atrial muscle at the superior caval vein exclusively resulted in an electrocardiographic $\mathrm{P}$-wave morphology which was identical to the morphology of a spontaneous heartbeat [154]. By using two recording electrodes Lewis also succeeded in localizing the region of earliest electrical activity or 'primary negativity' in the right atrium of the dog heart. This spot coincided with the histological location of the sino-auricular node [155]. Based on these and other electrophysiological findings Lewis [154-156] concluded that the pacemaker of the heart was located at the superior caval-auricular junction, corresponding with the position of the node of Keith and Flack [158]. In the same period Lewis' conclusions were confirmed by Eyster and Meek [74]. They also described that the point of primary negativity is usually located in the upper part of the node of Keith and Flack, but may shift to lower regions during vagal stimulation or injection of potassium.

In the next two decades several studies on the exact position, dimension and fiber types of the sinus node were published. Although in some papers, for example of Glomset and Glomset [84], the existence of a sino-auricular node was still denied, most studies confirmed the earlier findings of Keith and Flack, Lewis, and Eyster and Meek. It appeared that both the exact location and the size of the sinus node varled considerably among different species. The relative amount of nodal tissue was found to decrease and to become more concentrated in position when going from lower to higher vertebrates. However, also among animals of the same species a great individual variation in size and shape of the sinus node was recognized, as described by Taussig in 1933 [261]. The morphological description of the sinus node differed considerably among several authors: from “....a larger head lying within the epicardium near the superior vena cava, a tapering body, and more caudally a slender tall penetrating the atrial myocardium..." or "...a somewhat flattened central 
body with tapering ends..." to simply "...a horseshoe-shaped structura... " $[22,111$. 270].

The application of the microelectrode in experimental studies by Coraboeuf and Weidmann inaugurated a new era of sinus node and pacemaker physiology [111]. With this techniques it became possible to study the electrical characteristics of individual cells. In several studies the action potentials of sinus node fibers were de scribed. The phenomenon of spontaneous diastolic depolarization was soon recognized as the functional mechanism underlying spontaneous impulse formation $[51,66,267]$. In 1955 West expllored the isolated sinus node of the rabbit heart and demonstrated the suitability of this preparation to study pacemaker physiology [286]. In the following decades a great number of studies were performed on the electrophysiological properties of the rabbit sinus node. Different action potential configurations were described, and the sequence of excitation in the sinus was reconstructed by intracellular mapping $[23,32,35,101,171,204,205,225,253]$. It appeared that the pacemaker (earliest electrical activity) was not linked to a fixed position but could easily shift to other regions in the sinus node as a result of environmental changes $[29,30,35-37,164-166,264]$. This phenomenon was already suggested by Eyster and Meek in 1914 [74].

Recent electrophysiological and morphological studies revealed that two different regions can be distinguished within the sinus node including a central area and a perinodal border zone. In the isolated sinus node the center represents the functional region of impulse formation in which specialized pacemaker cells are found $[23,39]$. The transitional zone or bordér zone is situated in between the nodal center and the crista terminalis. This region functions as the sino-atrial conduction pathway and contains fibers which exhibit characteristics intermediate between typical pacemaker cells and atrial cells $[23,39,112,163,253]$. In the next sections the electrophysiological properties of both regions will be discussed in detail.

\section{Impulse formation in the sinus node}

From several studies it appeared that the cardiac impulse originates unifocally in the center of the isolated sinus node. Among other properties the fibers in this area are characterized by a steep spontaneous diastolic depolarization which is the functional mechanism underlying spontaneous impulse formation $[23,35,199,201,202$, 289 ]. In the isolated sinus node the site of impulse origin corresponds to the area where cells exhibit the steepest slope of spontaneous diastolic depollarization [23,35]. 


\section{Spontaneous action potential generation}

In the last decades it gradually became clear that the generation of action potentials in excitable cells is based on a complicated interplay of cellular membrane currents. In cardiac muscle fibers these currents are carried by several ions and during specific periods of the cardiac cycle. The properties of the cell membrane determine the kinetics of the individual currents and the electrophysiological profile of the cell $[39,104]$.

To measure the magnitude of these membrane currents and to study their physiological determinants, sophisticated voltage-clamp and patch-clamp techniques were developed and succesfully applied in isolated tissues [105]. The recent progress made in this field is both enormous and diverse, but till today no consensus exists on the basic mechanisms of action potential generation in cardiac fibers. In the present section a survey is given of the most important membrane currents which are believed to contribute to spontaneous impulse formation in the sinus node.

In principle, the voltage-clamp technique measures the total current which flows across the cell membrane when the cell is 'clamped' at a chosen potential. The contribution of specific ion-fluxes to this total current flow can only be determined indirectly by changing the electrolyte composition of the perfusion fluid or by pharmacological modulation of the membrane permeability for specific ions. In panel A of figure 1.1 typical action potentials of sinus node pacemaker fibers are depicted (upper tracing) together with the total current flow (I) across the cell membrane. The components of this total membrane current, deducted from the results of early voltage-clamp studies, are shown in the tracings below [104]. Four individual currentsystems were believed to contribute to the spontaneous generation of action potentials: (1) a time-dependent outward current ik, carried by $\mathrm{K}^{+*}$-ions and initially indicated as the primary component of spontaneous diastolic depolarization, (2) a slow inward current is, carried by $\mathrm{Ca}^{2+}$-ions and mainly responsible for the upstroke of the action potential; (3) a time-dependent slow inward current if ('funny current') of which the relevance was initially unknown; and (4) a time-dependent background current iL [44,65, 104,242,293,294].

In the most recent studies improved techniques yielded additional information on the relative contributions of the different membrane currents to spontaneous action potential generation [105]. In panel B of figure 1.1 the present view on pacemaker currents is depicted schematically. The decay of the $\mathrm{K}^{+}$-outward current (ik) after repolarization is believed to be responsible for the initial phase of diastolic depolarization. Subsequently, an inward $\mathrm{Ca}^{2+}$-current (iCa) promotes this diastolic depolarization which finally triggers the upstroke of the next action potentiall. In sinus 

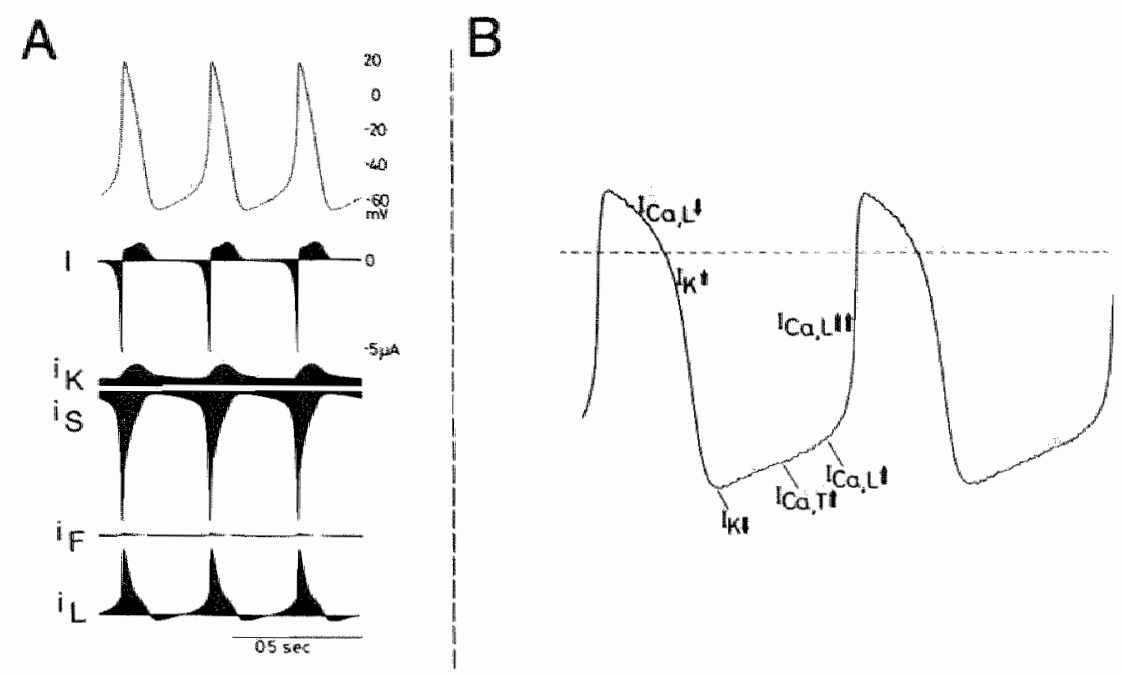

Figure 1.1:

Panel A: Reconstructed sinus node action potentials (upper tracing) and their composing current systems as elucidated by early voltage-clamp studies. The total membrane current (1) is mainly carried by an outward potassium current $i_{K_{n}}$ an inward calcium current $i_{S}$, the relatively small and controversal pacemaker current $I_{F}$, and by a background current $I_{L}$. (Reproduced from Irisawa and Noma 1982 [104], with permission of Martinus Nijhoff Publishers)

Panel B: Schematic representation of the contribution of different current systems to spontaneous impulse generation according to the findings of the most recent studles. The dilastolic depolarizathon is mainly based on a decay in $\mathrm{i}_{\mathrm{K}}$ and a successive increase in $\mathrm{I}_{\mathrm{Ca}_{\mathrm{R}}} \mathrm{T}$ and $\mathrm{i}_{\mathrm{Ca}, \mathrm{L}}$. (Reproduced from Irisawa and Hagiwara 1988 [105], with permission of Alan R. Liss, Inc.)

node pacemaker cells this inward $\mathrm{Ca}^{2+}$-current is composed of two specific components, indicated as the $T$ - and $L-t y p e(i \mathrm{Ca}, T$ and $\mathrm{iCa}, \mathrm{L})[105]$. The $\mathrm{L}-\mathrm{type} \mathrm{Ca}^{2+}$ current is characterized by an activation threshold of $-30 \mathrm{mV}$. This implicates that $\mathrm{iCa}_{\mathrm{C}} \mathrm{L}$ will only contribute to the last part of the spontaneous diastolic depolarization phase and to the upstroke of the action potential. The T-type $\mathrm{Ca}^{2+}$-current however, is characterized by an activation threshold of $-50 \mathrm{mV}$ and thus closes the gap between the decaying iK and the rising iCa,L $[39,94,105]$.

Other specific currents were suggested to contribute to spontaneous diastolic depolarization as well. One of these currents is the if or 'funny current'. The contribution of $i_{F}$ to the mechanism of spontaneous impulse formation is still unsettled. However, a dominant role seems unlikely because of its relatively slow time course of activation. Other relevant currents are the inward and outward background currents, suggested to be based partially on the $\mathrm{Na}^{+}-\mathrm{K}^{+}$pump and the $\mathrm{Na}^{+-}-\mathrm{Ca}^{2+}$ exchanger [105]. 
The presence of a typical central pacemaker area and a peripheral border zone illustrates the functional heterogeneity of the sinus node. This is reflected in the configurations of sinus node action potentials which change gradually when going from the center of the node towards the atrium $[23,171]$. The left part of figure 1.2 is taken from the study of Bleeker at al. [23]. Typical action potentials are shown, recorded from different locations in the isolated rabbit sinus node. Compared to fibers from the nodal center the cells in the periphery of the sinus node demonstrate a steeper action potential upstroke (see also section 'The electrophysiological characteristics of the transitional zone"). The steeper upstroke can be explained by the contribution of the fast sodium inward current $\mathrm{iNa}$, f to the phase-O depolarization in peripheral cells. This contribution is illustrated by the sensitivity of peripheral fibers to $T X$, an agent which blocks selectively the fast sodium inward current $[142,144,145,161$, 196,292]. With respect to automaticity, Kreitner found a marked reduction of the diastolic depolarization rate in fibers from the border zone after administration of caesium [145]. This was in contrast to the pacemaker cells from the center of the sinus node which appeared to be insensitive to caesium. Since caesium ions selectively block the if-current she concluded that this current does not flow in 'true' pacemaker cells [145].

Bouman and Jongsma [39] recently proposed the concept that spontaneous impulse formation in the nodal center occurs by virtue of the presence of if in the peripheral fibers. According to their view if allows the peripheral fibers to develop spontaneous diastolic depolarization which otherwise would be completely suppressed by the hyperpolarizing load of the attached atrium (see chapter II). Because of this diastolic depolarization in the peripheral cells the hyperpolarizing effect of the atrium does not reach the center of the sinus node. As a result, impulse formation in the nodal center is not electrotonically supressed by the load of the attached atrium although bath tissues are still electrically coupled.

\section{The site of impulse origin in the isolated sinus node}

To determine the site of impulse origin in the sinus node the sequence of excitation within the node must be reconstructed. in the isolated rabbit sinus node this is possible by the successive registration of multiple intracellular signals from many different sites in the node. However, this intracellular mapping technique is very time-consuming and only valid as long as the activation pattern and rate of discharge of the sinus node preparation remain unchanged. In larger species like dog and man, the sinus node is inaccessible to intracellular recording techniques because of the surrounding atrial myocardium [111]. 


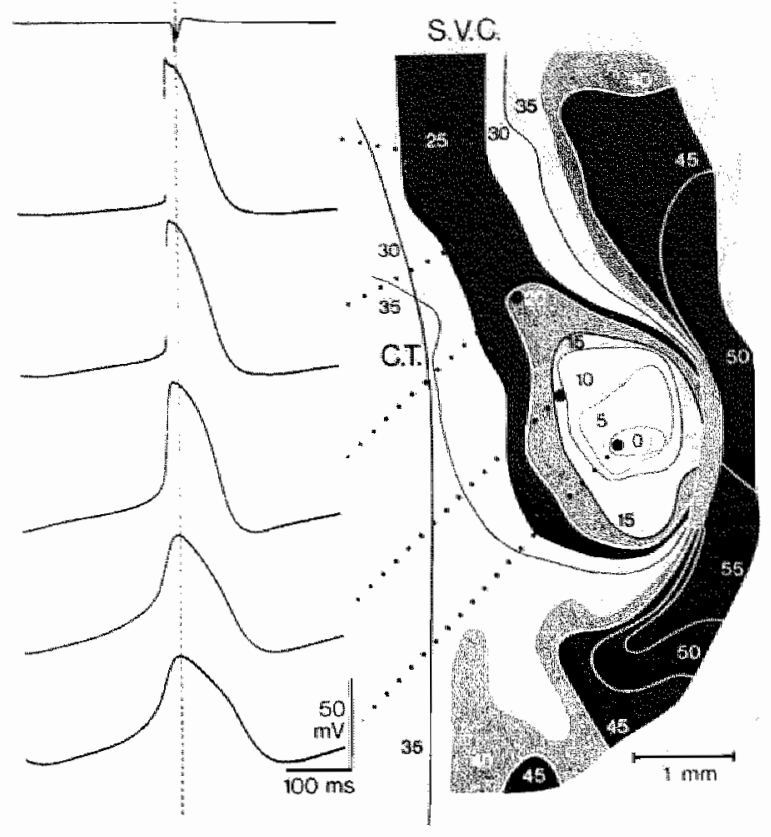

\section{Figure 1.2:}

Configuration of action potentials recorded in dif. ferent sites of the sinus node reglon. The se. quence of activation in the sinus nodie area is vis. ualized by isochrones of 5 ms. The area of earliest ectrical activity is repre sented by the 0-isochrone in the central portion of the sinus node. From this point the sinus impulse propagates through the transitional zone towards the crista terminalis. CT = crista terminalis: SVC = superlor vena cava. (Foproduced from Bleeker et all. 1980 [23], with per mission of the American Heart Assoclation)

Paes de Carvalho was one of the first investigators who mapped the spread of excitation in the isolated right atrium of the rabbit heart [205]. Some years later, in 1965, he was followed by Sano and Yamagishi [225] and by Bouman [35]. In these studies the earliest electrical activity was always found in the central portion of the sinus node in between the orifices of the caval veins at a distance of 1 to $2 \mathrm{~mm}$ from the crista terminalis. In this region always typical pacemaker action potentials were recorded. Later studies confirmed these findings in different species $[23,32,164,201$. 249]. In an extensive study on the functional and morphological organization of the isolated rabbit sinus node Bleeker and coworkers estimated the dimensions of the "primary" pacemaker [23]. In the right panel of figure 1.2 the reconstructed activation map of the sinus node region is given as found in one of their experiments. The site of earliest activation is indicated by the 0-isochrone. Within this 0-region a small area was identified which showed identical and synchronous electrical activity. This area covered about $0.1 \mathrm{~mm}^{2}$ and was estimated to contain about 5000 nodal cells. Morphologically this spot corresponded to the central portion. The typical ' $\mathrm{P}$ ' cells found in this region were characterized by a paucity of myofibrils, scattered small mitochondria, unorganized sarcoplasmatic reticulum and a clear or 'pale' cytoplasm 
$[23,112,114,163,171,258,262,268]$. The diameter of these relatively small and spindle shaped fibers varied between 3 and 9 micrometer.

In the isolated sinus node the location of the "dominant pacemaker is fixed as long as external conditions are stable. However, environmental changes often cause a change in pacemaker position within the sinus node. Usually, these pacemaker shifts are accompanied by a gradual change in sinus rate $[35,165]$. Lu $[164]$, Mackaay et al. [165,166], and Op "t Hof [200] described typical pacemaker locations during hypothermia, high $\mathrm{K}^{+}$, low $\mathrm{Ca}^{2+}$, low $\mathrm{Cl}$ and low $\mathrm{Na}^{+}$concentrations, and after addition of epinephrine or acetylcholine to the superfusion fluid. In case of low $\mathrm{Na}^{+}$, low $\mathrm{Cl}$, epinephrine and acetylcholine the pacemaker shifted even to the border zone of the sinus node. These results illustrated the functional inhomogeneity of the sinus node.

\section{The pacemaker in vivo}

The great number of studies in the isolated rabbit sinus node gained much insight into the basic mechanisms of cardiac pacemaking and sinus node functioning. However, an important limitation of the isolated preparation is the absence of the extrinsic regulatory systems which usually operate in vivo. Therefore, the functional characteristics of the sinus node in the intact animal were examined in several experimental studies $[25,85,116]$.

Recently, Boineau and colleagues [25-28] mapped the epicardial sequence of excitation at the junction of the superior caval vein and right atrium in anaesthetized dogs. An example of their results is shown in figure 1.3. Instead of a unifocal origin of the atrial depolarization wave, a multicentric complex of origins ( 3 to 6 origin points) was found along the epicardlal side of the crista terminalis. Depending on the actual heart rate always one of these points of origin demonstrated the earliest electrical activity. Each point dominated within a specific range of heart rates. At the trensition from one range to another this dominance showed a sudden shift between the neighbouring points of origin. A decrease in heart rate, due to alterations in autonomic balance, induced a caudal shift of the site of earliest activity allong the crista terminalis [25-28]. Histological examination demonstrated that the total area which was covered by this complex of pacemaker origins exceeded the boundaries of the sinus node [26]. The studies of Boineau and colleagues yielded important information on the cardiac pacemaker in vivo. However, two mechanisms may explain their findings. First, the multicentric complex of origins represents multiple individual pacemakers which discharge synchronously. The central ones are located within the sinus node whereas the peripheral ones are subsidiary atrial pacemakers [222]. 


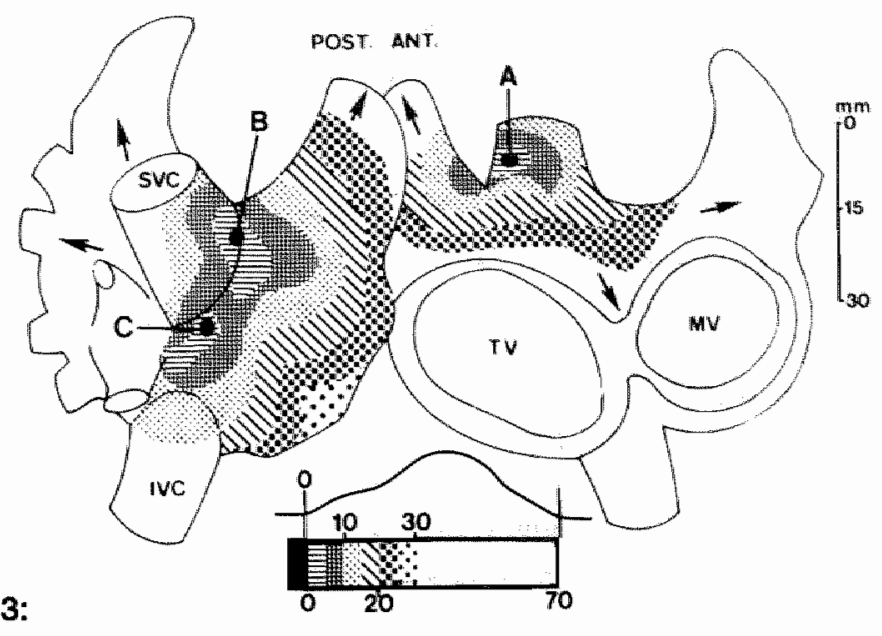

Figure 1.3:

Multicentric origin of the atrial activation wave during sinus rhythm in the intact dog. The activation pattern of the superior caval vein-atrlal junction is visualized by isochrones of $5 \mathrm{~ms}$. Note the simultaneous onset of eplcardial activation at three different points, indicated by $A_{t} B$ and $C$. $S V C=$ superior vena cava; $I V C=$ inferior vena cava; $M V=$ mitral valve; $T V=$ tricuspid valve. (Repro. duced from Boineau et al. 1980 [26], with permission of the American Physiolocal Society)

Second, in accordance to the prevailing theory of unifocal impulse formation, the multiple epicardial origins might be explained by the existence of preferential sinoatrial conduction pathways resulting in an almost simultaneous epicardial breakthrough of the sinus impulse at several locations. In this case intranodal pacemaker shifts will also change the site of earliest epicardial breakthrough [39]. Boineau and coworkers themselves favour the first concept: a complex system of isolated groups of pacemaker fibers modulated by the autonomic nervous system [25-28].

\section{Electrotonic interactions among pacemaker cells}

The theoretical concept of a single pacemaker fiber driving the heart seems unrealistic [35]. Even in the most profound studies this single fiber dominance could not be evidenced. Instead, Bleeker and colleagues [23] found that the sino-atrial pacemaker consists of a group of automatic fibers, all exhibiting synchronous and identical electrical activity.

Among others [57,58,117-119,295], Jalife, Michaels and coworkers recently studied the mechanisms underlying this pacemaker synchronization and developed the concept of mutual entrainment $[60,106,108-110,177-179,260]$. Between two or more pacemaker cells, each with a different intrinsic rate of discharge, the process of mu- 
tual entrainment is defined as a stable condition of electrotonic influences. This results in a synchronized and equal frequency of firing in all cells. Based on experimental findings with mathematical and biological models it was suggested that "...the origin and maintenance of the normal beat in the mammalian heart are based on a "democratic' process in which thousands of SA node cells, all capable of spontaneous activity, communicate with each other, reach a consensus, and discharge rhythmically and harmonically to initiate each propagated impulse." [110].

To obtain a quantitative description of mutual entrainment among automatic fibers, Jalife and coworkers used a mathematical model by which the phase-dependent effects of brief depolarizing current pulses on a single pacemaker fiber could be evaluated [109]. An example of their results is shown in figure 1.4. The behavior of a pacemaker fiber in response to depolarizing pulses was described by a phase-reponse relationship (panel C). Briefly, a subthreshold pulse delivered at incremental coupling intervals to the previous response will progressively delay the next response. Halfway of the cycle this delaying effect is suddenly replaced by an equivalent advancing effect which gradually decreases upon further lengthening of the coupling interval. Thus, depending on the phase difference to the preceding action potential a subthreshold pulse can either prolong or shorten the current cycle

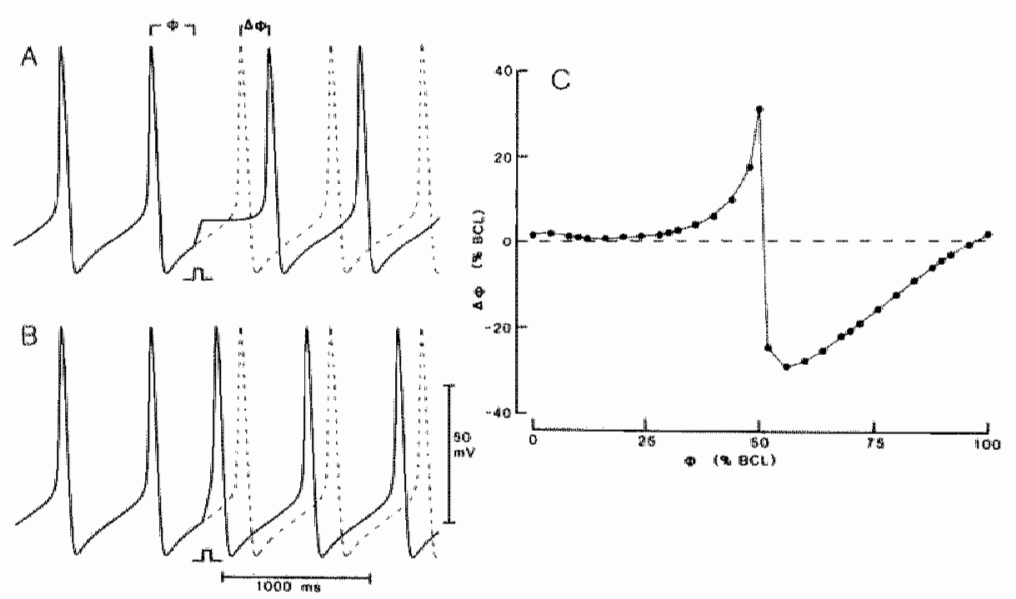

Figure 1.4:

Phase-dependent effects of depolarizing current pulses on simulated pacemaker cell activity. Application of brlef ( $50 \mathrm{~ms}$ ) subthreshold depolarizing current pulses delayed (panel A) or accelerated (panel $B$ ) the next discharge depending on the phase ( $\phi$ ) of the input. Panel $C$ shows a phase response curve summarlzing phase shifts $(\Delta \phi)$ produced by current pulses at various phases $(\phi)$ of the basic sinus cycle (BCL). (Reproduced from Jalife and Michaels 1985 [109], with permissilon of
Grune \& Stratton Inc.) 
length (panel A and B). This mathematical description was confirmed in isolated sinus node preparations of cat and rabbit hearts [106,109,228]. In the spontaneously firing sinus node the phase-response relationship exists among all electrically coupled pacemaker cells. Although these cells may have their own intrinsic rate of firing, the phase-dependent interactions were suggested to form the basis for the entrainment which leads to the synchronized discharge of all pacemaker cells in the sinus node [177]. The significance of mutual entrainment in pacemaker coordination and conduction within the sinus node was recently illustrated by Michaels et al. [178] using a mathematical model including multiple resistantly connected oscillating elements. Furthermore, the application of acetylcholine pulses was simulated and resulted in 'intranodal" pacemaker shifts similar to those observed in the isolated rabbit sinus node preparation. In their most recent study these authors demonstrate that repetitive vagal stimulation in random sites of the 'mathematical' sinus node may result in a regular, irregular or even chaotic sinus node response [179]. It was suggested that these vagally modulated changes in sinus node activity might be relevant under physiological conditions (baroreceptor reflex) and explain the occurrence of sinus arrhythmias [179].

The presence of low resistant intercellular connections or 'gap junctions' between pacemaker cells plays a crucial role in the achievement of synchronized automaticity. In several studies using computer models, single myocytes or aggregates of embryonic chick heart cells, it was found that individual pacemaker cells or cellgroups modulate each others rhythmicity as soon as they become electrically coupled $[50,57,58,60,108,117-119,177,271,276,295]$. The extent of this mutual electrotonic interaction was inversely related to the magnitude of intercellular coupling resistance $[60,117,177,295]$.

James and coworkers initially denied the presence of low resistant intercellular connections in the center of the sinus node [112]. However, recent electron microscopic studies demonstrated the presence of gap junctions between P-cells in the sinus node of the rabbit and other mammals $[23,170,199,201,202,266]$. In the center of the sinus node the incidence of gap junctions is lowest, but increases gradually towards the crista terminalis $[23,170]$.

\section{Autonomic nervous control of sinus node activity}

Stimulation of the parasympathetic nervous system reduces heart rate through the direct effects of acetylcholine on the pacemaker fibers in the sinus node [35-37]. Stimulation of the sympathetic nervous system causes the opposite by the release of catecholamines. In the isolated rabbit sinus node it was demonstrated that the 
chronotropic effects of acetylcholine and (nor)epinephrine are accompanied by changes in the site of impulse origin $[35-37,165,166,198,248,264,287]$. In the intact animal stimulation of the autonomic nervous system was shown to induce changes in the atrial sequence of excitation around the sinus node suggesting that pacemaker shifts also occur in the in vivo situation $[25,28,74,85]$.

Mackaay and coworkers extensively studied the mechanisms of autonomic frequency regulation in the isolated rabbit sinus node $[165,166]$. It was found that low concentrations of acetylcholine $\left(5.5 \times 10^{-6} \mathrm{M}\right)$ reduced sinus rate by $14 \%$ and caused a shift of the pacemaker to the caudal part of the nodal center. Higher dosages of acetylcholine $\left(13.8 \times 10^{-6} \mathrm{M}\right)$ decreased sinus rate by $22 \%$ but shifted the pacemaker in cranial direction into the border zone of the sinus node $[165,166]$. Apparently, acetylcholine suppressed the primary pacemaker cells in the nodal center to a greater extent than the subsidiary pacemaker cells in the periphery. As a result, the intrinsic rate of the cells in the center was reduced below the rate of the peripheral cells. Consequently, the latter took over pacemaker dominance. On the other hand, epinephrine $\left(6 \times 10^{-7} \mathrm{M}\right)$ increased sinus rate by $29 \%$ and shifted the pacemaker in caudal direction into the border zone $[165,166]$. This suggested that epinephrine accelerated the peripheral pacemaker cells to a greater extent than the leading pacemaker cells in the center of the sinus node [165].

The autonomic nervous system exerts a beat-to-beat modulating effect on the sinus node pacemaker $[35,38]$. Recentiy, Spear and coworkers demonstrated that brief vagal stimulation shifted the origin of the next sinus impulse and prolonged the current cycle length [248]. These effects were disappeared at the second sinus impulse after vagal stimulation. Jalife and coworkers confirmed these findings $[107,246]$.

\section{Sino-atrial conduction}

Paes de Carvalho [205], Bouman [35], and Sano and Yamagishi [225] found that the pacemaker in the isolated rabbit sinus node was located at a distance of 1 to $2 \mathrm{~mm}$ from the atrium (crista terminalis), and that the excitation of the sinus node region preceded the onset of the $\mathrm{P}$-wave in the surface electrocardiogram. These observations strongly suggested the existence of a phase of sino-atrial impulse conduction, which was confirmed by reconstruction of the entire excitation sequence of the isolated sinus node and right atrial appendage. However, no distinct bridge-like structure or specialized conduction tract could be identified between the center of the sinus node and the crista terminalis [225]. On the other hand, James found typical cells in between the central portion of the canine and human sinus node and the attached atrial myocardium [112]. Because of the location and histo- 
logical appearance these cells were indicated as transitional cells. Lowe et al. recently confirmed these findings [163].

Bonke and coworkers were one of the first who studied the characteristics of sino-atrial conduction by programmed electrical stimulation of the atrium [29]. In the isolated right atrium of the rabbit it was found that retrograde conduction of a premature atrial impulse into the sinus node was relatively slow. When the coupling interval of the premature stimulus to the foregoing sinus beat was gradually shortened, sino-atrial conduction became depressed [29]. Early premature atriall beats activated the sinus node only fractionally and were blocked before penetrating the pacemaking center of the node [30]. The results of these and other studies suggested an important role of the transitional fibers during sino-atrial impulse conduction $[29,30,35,95,225]$.

\section{The electrophysiological properties of the transitional zone}

In the early seventies Strauss and Bigger studied the basic electrophysiological properties of the transitional fibers in the isolated sinus node of the rabbit [253]. Multiple intracellular recordings revealed that the center of the sinus node was surrounded by a zone of cells exhibiting typical electrophysiological characteristics. Between the nodal center and the crista terminalis these perinodal fibers formed a discrete sino-atrial junction of about $0.5 \mathrm{~mm}$ in width. At the cranial, septal and caudall edges of the node the transitional zone narrowed.

The perinodal cells showed typical action potentials, characterized by “...(1) an amplitude and an upstroke velocity of phase 0 greater than those in sinus node cells, (2) little or no phase-1 (early rapid repolarization), (3) a phase-2 (plateau) longer than that seen in ordinary atrial fibers, which merged gradually with the rapid phase of repolarization (phase 3), (4) termination of phase-3 with a definite hyperpolarization, and (5) phase-4 (diastolic) depolarization slower than that in sinus node fibers" [253]. Spatial quantification of the different action potential parameters revealed that the membrane activation voltage, the maximal diastolic potential, the amplitude and, most pronounced, the slope of the upstroke all increased as function of the distance from the nodal center [24,253]. This suggested that impulse conduction in the perinodal zone accelerates from the center of the sinus node towards the crista terminalis. Besides this spatial quantification, Strauss and Bigger also studied perinodal action potential configuration during retrograde conduction of stimulated premature atrial depolarizations. It was found that the rate of depolarization decreased if the coupling interval of the premature atrial beat to the foregoing sinus activation was shortened. This explained the earlier findings of Bonke and cowor- 
kers [20,30]. Strauss and Bigger concluded that the perinodal zone serves as the site of normal conduction delay for impulses entering and leaving the sinus node and that the perinodal region is a preferential area for the occurrence of sinus exit block [253].

Another important aspect of their findings was the moderate rate of spontaneous diastolic depolarization in perinodal fibers. In principle this implicates the possibility of pacemaker activity. However, Strauss and Bigger never obtained evidence for self-excitation in these cells. In their experiments the intrinsic rate of firing of the transitional fibers was always lower than rate of pacemaker fibers in the center of the sinus node, even at sinus rates as low as 60 beats per minute [253]. The authors concluded that the perinodal fibers could not incorporate the sinus pacemaker. This was contradicted by the results of recent studies (see also chapter II) which demonstrated that the fibers in the border zone, under certain conditions, can take over pacemaker dominance and act as the 'leading' pacemaker of the heart $[36,37,165$, $166,250]$. In previous sections this issue was discussed in greater detail.

\section{Morphological organization of the transitional zone}

In the study of Bleeker et al. the electrical properties of the perinodal zone were correlated to the morphological characteristics [23]. Morphological examination revealed tissue continuity at all levels between the center of the node and the crista terminalis. In contrast to earlier reports of James [113] and Sano and lida [227] no specialized sino-atrial conduction tracts were found. Ultrastructurally , there was a gradual transition in cell type from the typical P-cells in the nodal center to the ordinary atrial cells in the crista terminalis. The dimensions of the transitional cells were comparable to those of the P-cells. Towards the atrium the shape and arrangement of the transitional cells became more regular and parallel to the crista terminalis. The myofilaments became better organized and increased in amount. Gap junctions were observed more frequently and increased in size. These histological findings confirmed those of other studies $[102,170,266]$.

The activation maps and intracellular registrations (figure 1.2) as obtained by Bleeker and coworkers [23] showed that conduction of the impulse in the transitional zone accelerates in the direction of the crista terminalis as suggested by Strauss and Bigger [253]. However, Bleeker and colleagues did not interpret the transitional zone as the site of normal conduction delay for impulses leaving the sinus node, but rather as the electrophysiological and morphological transition from primary pacemaker cells to fast conducting atrial fibers. 


\section{Assessment of sino-atrial conduction in vivo}

In the isolated sinus node preparation both antegrade and retrograde sino-atrial conduction can be studied by intracellular recording techniques. However, in larger animals and in man this is not possible. In these species sino-atrial conduction can only be estimated indirectly by using catheter electrodes and programmed electrical stimulation of the right atrium. Clinically, these techniques are used as a diagnostic tool in patients with suspected sinus node dysfunction $[75,76,87,96,97$, 212,213].

Recently, direct sinus node electrographic techniques have become available to study sinus node function in vivo $[54,88,96,97,213,216]$. By means of catheter electrodes positioned in the high right atrium, extracellular activity can be recorded which preceeds the onset of the P-wave during sinus rhythm and which is interpreted as early sinus node activity. Compared to the conventional techniques this direct sinus node electrography allows a more reliable evaluation of sinus node automaticity and sino-atrial conduction, and can be used to establish sinus node dysfunction [88,216]. On the other hand, it must be stressed that in larger species like dog and man the sinus node is covered by atrial myocardium. As a consequence, the sinus node electrogram probably shows the earliest atrial breakthrough of the sinus impulse rather than actual sinus node pacemalker activity $[25-28,39]$.

In vivo the sino-atrial conduction time (SACT) can be determined by premature atrial stimulation according to Strauss et'al. [254], or by constant atrial pacing as proposed by Narula et al. [191]. In principle, both techniques measure the sinus return cycle after retrograde conduction of a single premature atrial impulse (Strauss) or a train of atrial impulses (Narula) and interpret the sinus node return cycle as the sum of the spontaneous sinus cycle and the antegrade and retrograde conduction time between the nodal center and the atrium. In figure 1.5 the measurement of the SACT in man by the Strauss technique is illustrated [254]. The diagram shows the normalized sinus return cycle as function of the normalized premature cycle. Atrial impulses delivered in late diastole collide in the sino-atrial border zone with the antegrade conducting sinus impulse and result in a complete compensatory pause $\left(A_{1}-\right.$ $\left.A_{2}+A_{2}-A_{3}=2 A_{1}-A_{1}\right)$, indicated as zone 1 in the diagram. When the prematurity of the atrial extrasystole is increased it will, at a certain point in diastole, penetrate the center of the sinus node and activate the pacemaker cells prior to spontaneous discharge. This pacemaker reset results in a non-compensatory pause $\left(A_{1}-A_{2}+\right.$ $A_{2}-A_{3}<2 A_{1}-A_{1}$ but $\left.A_{2}-A_{3}>A_{1}-A_{1}\right)$, indicated as zone II. At this point the difference between the return cycle $\left(A_{2}-A_{3}\right)$ and the spontaneous cycle length $\left(A_{1}-A_{1}\right)$ represents the sum of the retrograde and antegrade conduction time (SACTA+R) of the premature atrial impulse and the subsequent sinus impulse respectively. To 


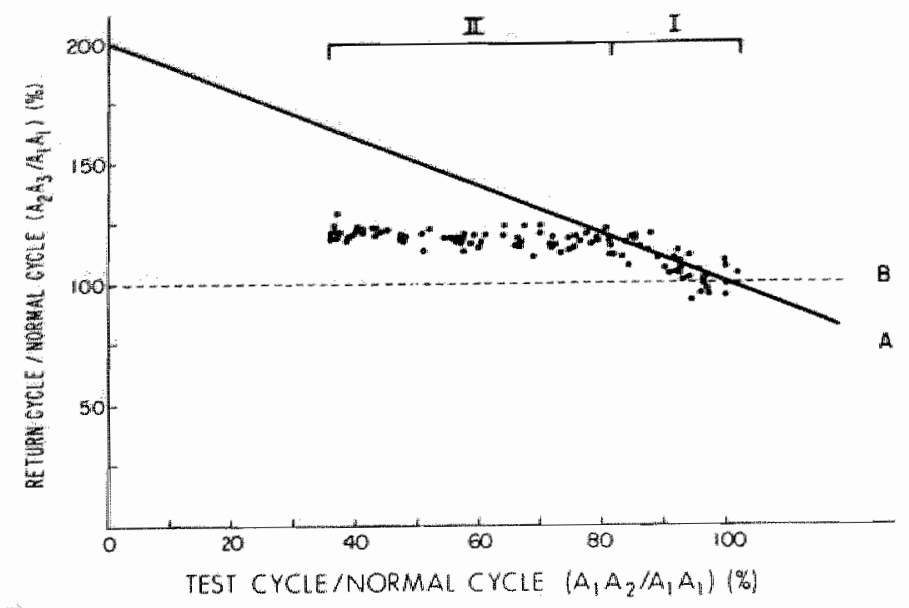

\section{Figure 1.5:}

Estimation of $S A C T_{A+R}$ in man. The normalized return cycle (ordinate) is plotted as function of the normallzed premature cycle (abscissa). Atrlal premature beats elicited in zone I are compensatory as indicated by the compensatory line A. Atrial premature beats elicited earlier in diastole, indicated as zone II, fall below the compensatory line and form a plateau. The average return cycle of the latter pert of zone Il represents the SACT $_{A+A}$. (Reproduced from Strauss et al. 1973 [254], with permission of the American Heart Association)

calculate the singular SACT, the average $S A C T A+R$ of the latter one third of zone II is divided by 2 .

This indirect estimation of the SACT is based on three assumptions: (1) a premature atrial beat which resets the sinus node does not change sinus rate; (2) the early atrial depolarization does not induce a pacemaker shift within the node; (3) the antegrade sino-atrial conduction time equals the retrograde sino-atrial conduction time. However, experimental evidence invalidates these assumptions and emphasIzes carefull interpretation of the estimated SACT $[29,30,39,138,180,214,249]$.

Narula and coworkers suggested to measure the sinus node return cycle after a period of atrial pacing [191]. This method does not improve the accuracy of the premature atrial stimulation method, whereas phenomena like sinus node reentry and interpolation of atrial premature beats can not be studied [91]. On the other hand, the 'Narula-technique" enables estimation of the SACT in patients with sinus arrhythmias [216]. 


\section{Sick Sinus Syndrome}

Normal sinus node function requires adequate impulse formation followed by undisturbed conduction of the impulse towards the atrial myocardium. If one or both mechanisms are impaired the sinus node will intermittently or permanently fail to activate the atrium. In patients sinus node dysfunction must be deducted from the surface electrocardiogram. In the past, different kinds of arrhythmias were recognized as pointing to sinus node dysfunction. These were grouped together by Ferrer [75] as part of the 'Sick Sinus Syndrome'. The sick sinus syndrome may manifest itself by one or more of the following findings: (1) persistent, severe and unexpected sinus bradycardia; (2) cessation of sinus rhythm for shorter or longer periods (sinus 'pause' or sinus 'arrest'); (3) inability of the heart to resume sinus rhythm after cardioversion from atrial fibrillation; (4) chronic atrial fibrillation with a slow ventricular response; (5) episodes of sino-atrial exit block unrelated to drug therapy; (6) the occurrence of the typical bradycardia-tachycardia syndrome [67,75,212].

Sinus node dysfunction might only be present intermittently or show a complex electrocardiographic pattern due to of subsidiary pacemaker interference. In both cases provocative testing by programmed electrical stimulation is required to disclose disturbances in sinus automaticity or sino-atrial conduction. Silnus automaticity can be evaluated by determination of the sinus node recovery time (SNRT), which is the time interval between the last stimulated beat and the first sinus escape beat after a period of atrial overdrive pacing [122,239]. Since the duration of the SNRT depends on the normal sinus cycle length, the SNRT is corrected for this factor by subtracting the basic sinus cycle length from the SNRT. The longer the corrected SNRT, the greater the degree of sinus node suppression. On the other hand, it must be emphasized that the corrected SNRT not only depends on sinus automaticity but also on sino-atrial conduction. This can lead to overestimation of the (c)SNRT $[239,255]$. In 35-78\% of the patients with 'known' sick sinus syndrome the (c)SNRT is significantly prolonged and might be usefull as a diagnostic tool $[39,76,93,168,189$, $212,255]$.

Conduction of the impulse through the sino-atrial junction can be tested by indirect estimation of the sino-atrial conduction time [191,254]. This technique as well as its limitations have been described in more detail in the previous section. The estimated SACT is less sensitive than the (c)SNRT to detect sinus node dysfunction $[40,255]$. In addition to the SACT the effective refractory period in the sinus node can be estimated by the 'Strauss' or 'Narula' technique as well [134]. At progressively shorter coupling intervals of premature atrial depolarizations the transition from sinus reset (zone II in figure 1.5) to interpolation reflects sino-atrial refractoriness. 
Kerr et al. recently demonstrated that measurement of the sino-atrial refractory period might by usefull as a diagnostic tool in suspected sinus node dysfunction [136].

Sinus dysfunction can be acute, paroxysmal or chronic. It may be due to an intrinsic disease of the sinus node, to electrolyte imbalance, to drugs or malfunction of the autonomic nervous system [15,122]. It is most frequently found in the elderly but can also occur in children and newborns $[19,217,291]$.

\section{Sinus node reentry}

The sinus node might favour the occurrence of arrhythmias when it becomes the slow conducting element of a reentrant circuit [2-4]. This can lead to single sinus echo beats or sinus node reentrant tachycardias [49,56,206,215,256,287].

In clinical studies short post-extrasystolic return cycles and high right atrial tachycardias were described which demonstrated an atrial activation sequence similar to that of normal sinus beats $[14,42,46,49,56,81,206,209,215,279]$. In principle, short post-extrasystolic responses may be explained by different mechanisms $[89,176]$. Experimental studies in dogs however, indicated that these arrhythmias are based on sinus node reciprocation or sinus node reentry if the following indirect criteria are fulfilled: (1) initiation of the supposed sinus echo beat or sinus node reentry by a properly timed premature atrial beat $\left(\mathrm{A}_{2}\right)$; (2) reproducible from different stimulation sites; (3) an $A_{1}-A_{3}$ interval remarkably shorter than the spontaneous cycle length $\left(A_{1}-A_{2}+A_{2}-A_{3}<A_{1}-A_{1}\right) ;(4)$ a $P$-wave morphology similar to that of sinus beats; (5) an $A_{3}-A_{4}$ cycle length longer than the $A_{1}-A_{1}$ interval (sinus reset) to exclude sinus entrance block; (6) exclusion of a right-sided accessory bypass tract which can mimic a high-to-low activation pattern of the right atrium $[14,42,43,46,49,56,190$, $\left.197,206,208_{1} 209,215,279,290\right]$.

Recently, sinus node reentry was found in patients with apparent sinus node dysfunction $[49,56,207,215]$, but also in healthy subjects $[42,206,209,284,290]$. Dhingra et al. reported an $11 \%$ incidence after premature atrial beats which were evoked during sinus rhythm, and a $26 \%$ incidence after premature depolarizations which were induced during atrial pacing [64]. Wellens reported a $9.4 \%$ incidence of sinus node reentrant beats during programmed electrical stimulation, being about one third of the incidence of AV nodal reentrant beats [285]. Josephson found a $4 \%$ incidence of sinus node reentry [124].

Despite of indirect evidence obtained in experimental and clinical studies, direct demonstration of sinus node reentry is hardly possible. Only in a few experimental studies using isolated rabbit sinus node preparations direct evidence for the occurrence of sinus node reentry could be obtained $[5,95,256]$. The first of these studies 
was performed by Han, Malozzi and Moe in 1968 [95]. In figure 1.6 part of their results are reproduced. In the upper left corner of the figure a schematic representation of the preparation is given. The different extra- and intracellular recording sites are indicated. During slow pacing $\left(S_{1}\right)$ the impulse propagated from the point of stimulation (S) to fibers $\mathrm{Aa}, \mathrm{Ab}, \mathrm{SNa}, \mathrm{SNb}$, and $\mathrm{SNc}$ in that order. However, during conduction of an early atrial impulse $\left(\mathrm{S}_{2}\right)$ the order of activation was changed. Now fiber SNb was activated first, followed by SNa. The area of fiber SNc was not activated at all. The impulse left the sinus node somewhere in the area of fiber $\mathrm{SNa}$ and reentered the atrium. Subsequently, the impulse invaded the sinus node again,

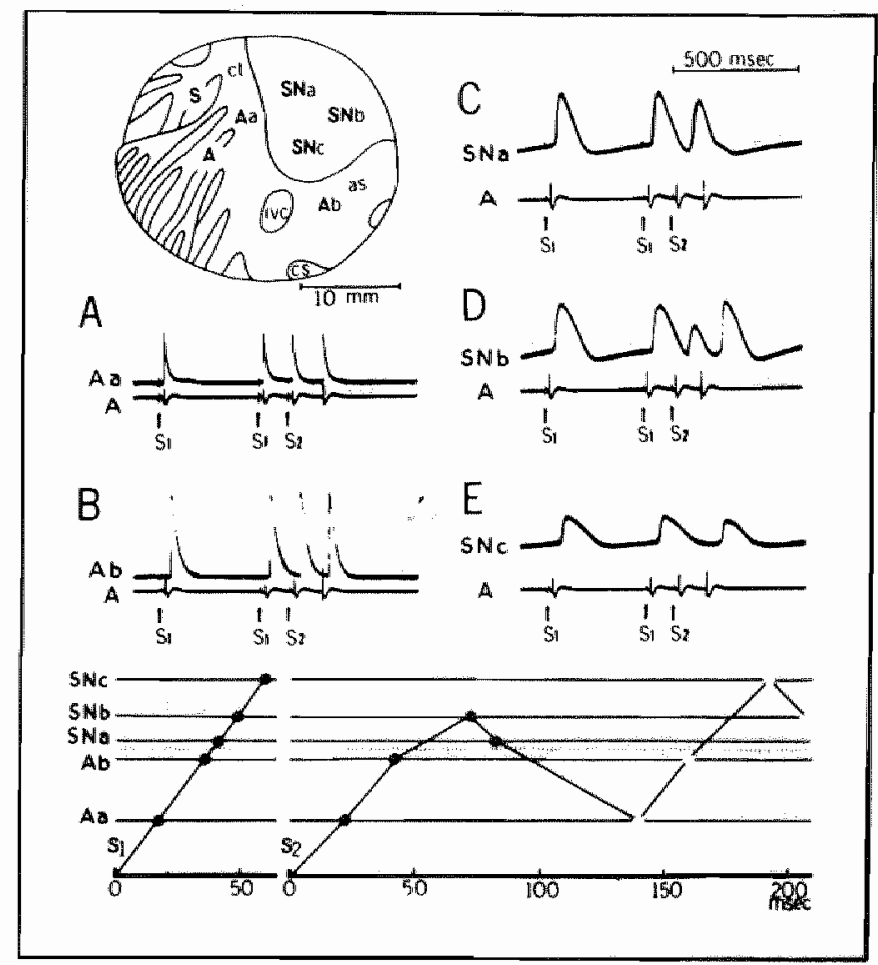

\section{Figure 1.6:}

Rieconstructlon of sino-atrial echoes. Locations of the electrodes ara shown in the schematic drawIng of the preparation on the upper left. The intracellular recordings were obtained from sites $\mathrm{Aa}$, $A B, S N a, S N b, S N c$, the local electrogram from site $A$. The responses in each recording site are shown in panels $A$ to $E$. The bottom graph shows the temporal sequence of activation in these sites during the basic stimulus $S_{1}$ and the premature stimulus $S_{2}$ which induced the echo beat. (Reproduced from Han et al. 1968 [95], with permission of the American Heart Association) 
but now at the region of fiber SNC, and extinguished somewhere in the sinus node. These findings suggested that during sinus echo beats the occurrence of local sinoatrial entrance block (SNC) induced a circumferential excitation of the sinus node followed by reactivation of the atrium at the previous site of entrance block. However, the authors stated themselves that "Definitive proof of a circuit within the sinus node would require a number of simultaneous impalements of intranodal cells, and a point-to-point demonstration of the course of the activation front during the whole interval between primary and reentrant atrial responses" [95].

Simultaneous intracellular mapping of the isolated sinus node is still a technical illusion. Only Allessie and Bonke recently succeeded in reconstructing the activation pattern of the sinus node during the repetitive induction of a sinus echo beat [5]. Therefore a large number (130) of intracellular recordings were obtained from the sinus node region. The sequence of excitation in the crista terminalis and right atrial appendage was simultaneously mapped by 192 unipolar surface electrodes. The results of this experiment are shown in figure 1.7. In the left panel a selection of 16 intracellular recordings is given together with the atrial reference electrogram during sinus rhythm and an induced sinus echo beat. In each signal the moments of excitation are given related to the moment of earliest activation (sinus rhythm) or electrical stimulation (premature atrial beat). In the right panel the respective intracellular recording sites (upper diagram) and the reconstructed conduction route during the sinus echo beat (lower diagram) are shown. It was found that the premature atrial depolarization wave encountered a local sinus entrance block in the caudal part of the preparation. After slow circumferential conduction through the sinus node this area of entrance block was retrogradely activated and followed by reexcitation of the atrium. Thus far this confirmed the suggestions of Han et al. [95]. However, in this experiment the premature impulse first described an intranodal reentrant loop before leaving the sinus node. Although the second detour was only partially accomplished this definitely demonstrated the possibility of an intranodal reentrant loop.

\section{The sinus node and atrial fibrillation}

Atrial fibrillation is primarily based on functional reentrant excitation whereby muitiple independent activation waves propagate randomly through the atrial myocardium [6,182-185]. As long as a sufficient number of wavelets are present the fibrillatory process will perpetuate $[6,8]$.

Based on indirect experimental evidence it has been suggested that the sinus node might play a facilitating role in the genesis and perpetuation of the atrial fibril- 

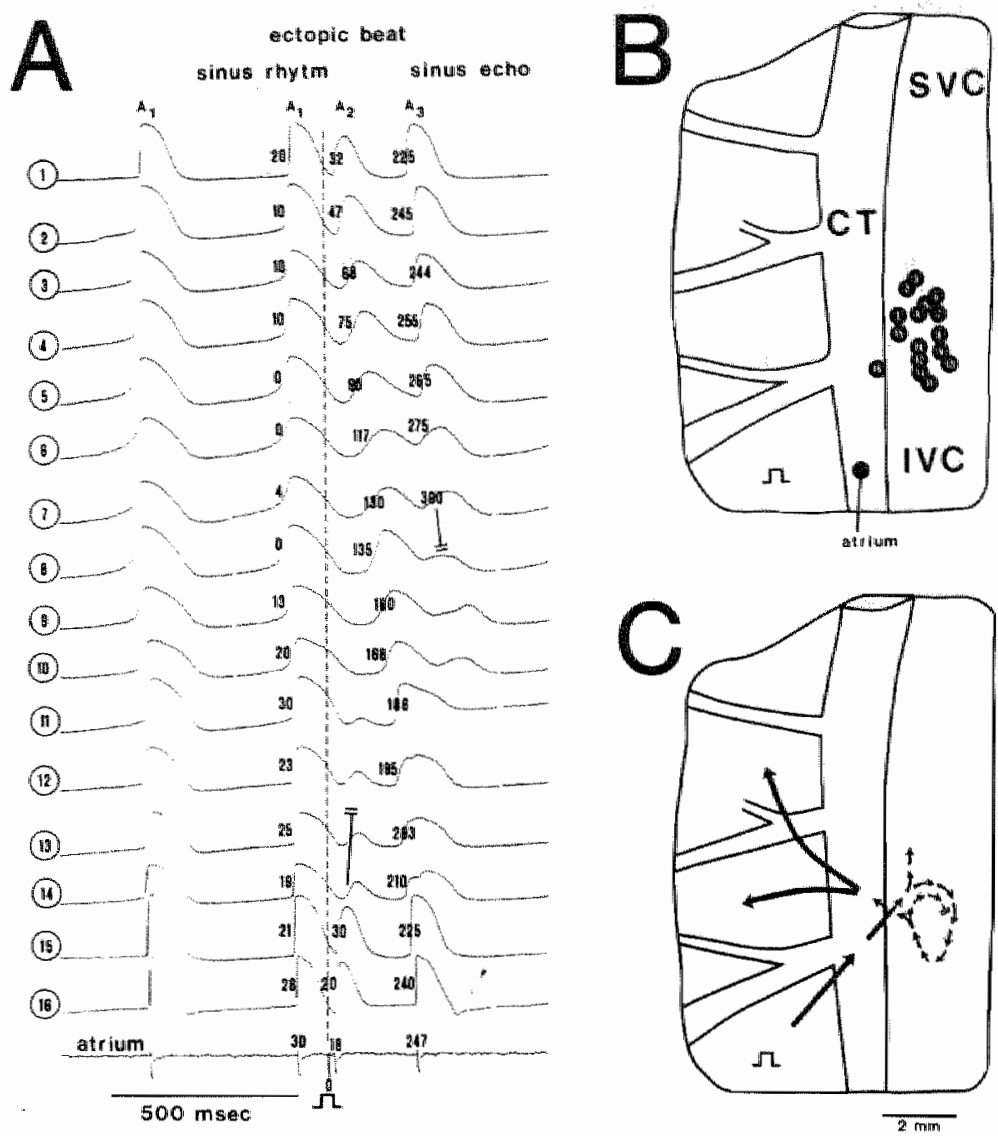

\section{Figure 1.7:}

Panel A: Transmembrane potentlals of 16 different flbers in the sinus node together with an atrial reference electrogram. The different intracellular recordings. were not obtained simultaneously but were time aligned to the reference electrogram which showed a constant response. The first two action potentials represent the last two discharges of a serles of 15 sinus beats. After the $15^{\text {th }}$ sinus beat an early premature stimulus was applied to the atrlum (dotted line). Retrograde invasion of the ectopic beat in the sinus node was blocked in the area of flbers 11-14. However, in the upper part of the node (fibers 1-7) the early premature impulse succeeded to penetrate the node. The impulse was conducted along a pathway from fiber 1 to 2 to 3 to 4 to 5 etc (panel $\mathbb{B}$ ). The fibers which initially were not activated directly by the retrograde wavefront (fibers 8-14), now yet were excited in a roundabout way. So, $225 \mathrm{~ms}$ after the stimulus, the impulse returned at the site where it entered the node (fibers 1 and 15 ). By this time these fibers had recovered their excltability and were reex. cited. Shortly thereafter the impulse left the sinus node agaln at about the same point where it had entered and the atrium was reexcited as weil. In panel $\mathrm{C}$ a schematic representation is given of the circultous pathway of the limpulse within the slnus node. (Reproduced from Allessle and Bonke 1979 [5], with permission of the American Heart Association) 
tion or functional suppression of the sinus node reduced the occurrence and stability of fibrillation $[13,187,192,226]$. Another argument in favour of a role of the sinus node in atrial fibrillation is given by the increased incidence of this arrhythmia in patients with sick sinus syndrome $[75,115,212]$.

Recently, Allessie and coworkers reconstructed the atrial activation pattern during atrial fibrillation in the dog heart $[6,8]$. The results of this study confirmed Moe's multiple wavelet theory of fibrillation. However, in these experiments no spontaneous activity arising from the sinus node region could be identified. On the other hand, it could also not be excluded. One of the purposes of the present thesis was to investigate the role of the sinus node in the process of atrial fibrillation. 


\section{Chapter II}

THE INFLUENCE OF THE ATRIAL MYOCARDIUM ON IMPULSE FORMATION IN THE RABBIT SINUS NODE 



\section{Introduction}

Recent electrophysiological and morphological studies demonstrated the functional inhomogeneity in the isolated rabbit sinus node. A central pacemaker area was distinguished which was surrounded by a transitional zone or border zone $[23,145,253]$. The border zone forms the connection between the center of the sinus node and the atrial myocardium (crista terminalis). In the isolated rabbit sinus node the site of impulse formation is normally located in the center where typical pacemaker fibers ( $\mathrm{P}$-cells) are found $[23,32,225]$. However, this pacemaker position is not fixed and can easily shift to another location within the node. Mostly these pacemaker shifts are accompanied by a simultaneous change in sinus rate. These changes in the site of impulse origin indicate that spontaneous impulse generation is not an exclusive feature of one particular group of sinus node fibers, but may be taken over by other fiber groups within the node. Environmental changes, drugs, or autonomic transmitters might alter this intranodal pacemaker hierarchy and thus modulate sinus thythm $[36,37,165,250,264]$.

In recent studies, using mathematicall models, cultured cell aggregates or small sinus node specimen, it was found that electrically coupled pacemaker fibers or fiber groups modulate each others rhythmicity by electrotonic interactions and thus accomplish synchronized firing $[57,58,92,108,109,271]$. The extent of this mutual electrotonic influence is related to the amount of intercellular coupling resistance among the pacemaker fibers $[60,117,295]$. Several investigators emphasized the relevance of this phenomenon in the coordinated process of pacemaking within the sinus node $[12,31,141,145,228]$.

However, sinus node pacemaker fibers are not only coupled to each other but also in their entirety to a relatively thick bundle of atrial myocardium, the crista terminalis. It was the goal of the present experiments to study the influence of this rela tively thick atrial bundle on impulse formation in the isolated rabbit sinus node.

\section{Methods}

\section{The preparation}

Young New Zealand rabbits of either sex and weighing 1.5-2.0 kg were killed by cervical dislocation. After midsternal thoracotomy the pericardium was opened and the heart quickly excised. After isolation of the right atrium a preparation was made including the roof of the right atrial appendage, the crista terminalis, and the inter- 
caval tissue which incorporates the sinus node. The AV node and interatrial septum were removed. The final preparation was pinned upon a silicon bar and placed in a tissue-bath $(35 \mathrm{ml})$ with its endocardial side facing upward. The tissue-bath was continuously perfused with oxygenated Tyrode solution $(100 \mathrm{~m} / / \mathrm{min})$ of $37 \pm 0.2^{\circ}$ Celsius. The solution contained in $\mathrm{mM}$ : $\mathrm{NaCl} 130, \mathrm{KCl} 5.6, \mathrm{CaCl}_{2} 2.2, \mathrm{MgCl}_{2}$ 0.6, $\mathrm{NaHCO}_{3} 24.2, \mathrm{NaH}_{2} \mathrm{PO}_{4} 1.2$, glucose 11 and sucrose 13. It was saturated with a mixture of $95 \% \mathrm{O}_{2}$ and $5 \% \mathrm{CO}_{2}$. The $\mathrm{pH}$ was $7.35 \pm 0.05$.

After the isolation procedure no measurements were performed during at least 30 minutes. Preparations demonstrating sinus arrhythmias were rejected. After complete stabilization of sinus rhythm the site of impulse formation and the spread of excitation in the sinus node were reconstructed. Subsequently, the sinus node and atrial myocardium were separated whereafter the nodal activation pattern was mapped again.

\section{Experimental protocol}

During the experiments a bipolar surface electrogram was continuously monitored. The recording electrode consisted of teflon-coated silver wire $(0.25 \mathrm{~mm}$ diameter, $0.3 \mathrm{~mm}$ interpolar distance), and was positioned on top of the crista terminalis. By means of standard microelectrode techniques (glass capillaries filled with $3 \mathrm{M} \mathrm{KCl}$ and a tip resistance of 10-30 $\mathrm{MOhm}$ ) multiple intracellular recordings ( $n=80-120 ; 0.3 \mathrm{~mm}$ spatial resolution) were obtained from fibers in the sinus node region to reconstruct the nodal sequence of excitation. The moment of activation of the impaled fibers was defined as the time difference between the action potential upstroke ( $50 \%$ level of the amplitude) and the intrinsic deflection of the atrial surface electrogram. This time difference was measured by a digital counter (Hewlett Packard $5300 \mathrm{~B}$ ). The area where fibers showed the earliest moment of activation was indicated as the site of impulse formation. This point was designated as time moment zero. The activation pattern in the sinus node was reconstructed by relating the local activation times to the site of earliest activity. The intracellular mapping technique is only valid as long as sinus rhythm remains constant and the pacemaker location fixed during the time period necessary to produce the activation map (2-3 hours). These prerequisits were continuously checked by measurement of the spontaneous cycle length and consideration of the shape of the atrial electrogram. Changes in one or both parameters could be due to an intranodal pacemaker shift. In that case the mapping procedure was restarted or the entire experiment rejected. All signals were stored on magnetic tape (Ampex PR 2200) for off-line analysis. 
After reconstruction of the nodal activation sequence the transition between the crista terminalis and the border zone of the sinus node was determined through a dissecting microscope (magnification factor: $16 \mathrm{x}$ ), and by means of intracellular recordings. At the transition between atrium and border zone the tissue exhibited a less reddish appearance, whereas the local action potential configuration became less atrial-like. Subsequently, the crista terminalis and connecting atrial appendage were separated from the sinus node by an incision along the sino-atrial transition. The incision was made by a fine pair of scissors. After sino-atrial separation the surface electrode was repositioned at the edge of the nodal preparation. Monitoring of spontaneous activity and continuous measurement of the spontaneous cycle length was restarted immediately. After a stabilization period of 30 minutes the location of the pacemaker and the activation pattern in the sinus node were mapped again. The microelectrode impalements were made in the same locations as before the sinoatrial disconnection. Thus it was possible to compare the site of impulse origin, the sequence of excitation, and the spatial variation in action potential configuration in the sinus node before and after removal of the crista terminalis.

\section{Results}

\section{Sinus rate before and after disconnection of atrium and sinus node}

In 15 experiments the crista terminalis was separated from the sinus node to study the passive influences of the atrial myocardium on the sinus node. Under control conditions all preparations demonstrated a stable sinus rhythm with an average spontaneous cycle length of $348 \pm 50 \mathrm{~ms}$ (mean $\pm \mathrm{SD}$ ). Two of these preparations showed a relatively high sinus rate ( 260 and $265 \mathrm{~ms}$ interval). Reconstruction of the nodal activation pattern revealed that in these two cases the pacemaker was located in the border zone of the sinus node instead of in the nodal center as found in the other cases. Without these two extraordinary preparations the average spontaneous cycle length was $361 \pm 38 \mathrm{~ms}$. After determination of the pacemaker Iocation and reconstruction of the nodal activation sequence, the crista terminalis was disconnected from the sinus node. All preparations with the pacemaker located in the nodal center showed an immediate increase in sinus rate after removal of the atrium. This phase of sinus acceleration lasted 10-20 minutes after which the spontaneous cycle length stabilized and remained equal during the further course of the experiment (about 4 hours). After 1 hour the average sinus interval in all preparations was $294 \pm 42 \mathrm{~ms}$, being a significant decrease of $18 \%(p<0.001)$ in comparison to control. Figure 2.1 depicts the individual changes in sinus interval sche- 


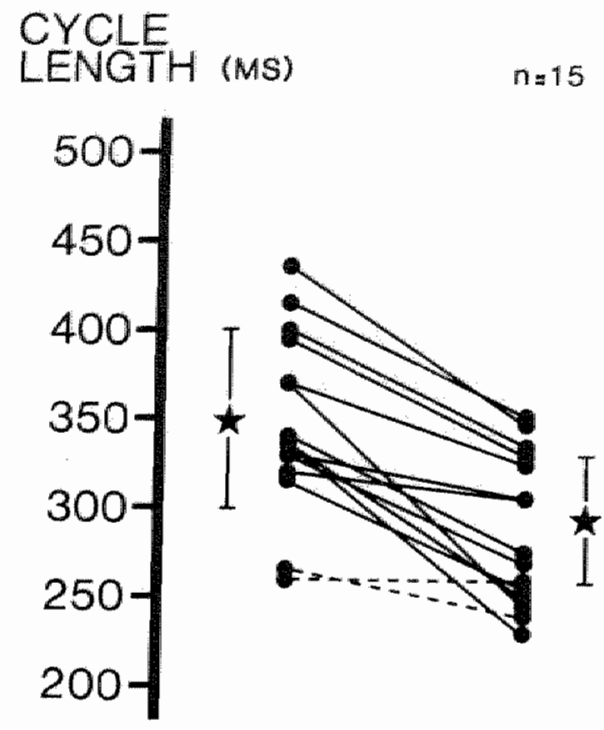

Figure 2.1:

Changes in spontaneous sinus interval after sino-atrial disconnection. The results of each experiment are shown. The mean values of all experiments $(n=15)$ are represented by asterisks. The two preparations which showed a relatively high sinus rate during control are indicated by dotted lines.

\section{CONTROL AFTER $X_{0}$}

matically. The two preparations which showed a relatively high sinus rate during control are indicated by dotted lines.

\section{Sinus node activation pattern after disconnection from the atrium}

In figure 2.2 a typical example is given of the activation sequence in the sinus node before and after separation from the atrium. The upper left part of the figure shows the control map. The sequence of excitation is visualized by isochrones of $10 \mathrm{~ms}$. The site of earliest activation (asterisk) was found at a distance of about $0.7 \mathrm{~mm}$ from the crista terminalis, which means in the central portion of the sinus node. From this point the impulse propagated very slowly through the nodal center towards the border zone. In the border zone it accelerated and conducted preferentially in a cranial direction towards the crista terminalis, the latter being activated after $20 \mathrm{~ms}$. The activation patterns of the other 12 preparations with the pacemaker located in the nodal center was similar. On the average the pacemaker was found at a distance of $0.6 \pm 0.12 \mathrm{~mm}$ from the sino-atrial transition. The average moment of activation at the crista terminalis was $21 \pm 11 \mathrm{~ms}(n=13)$. 

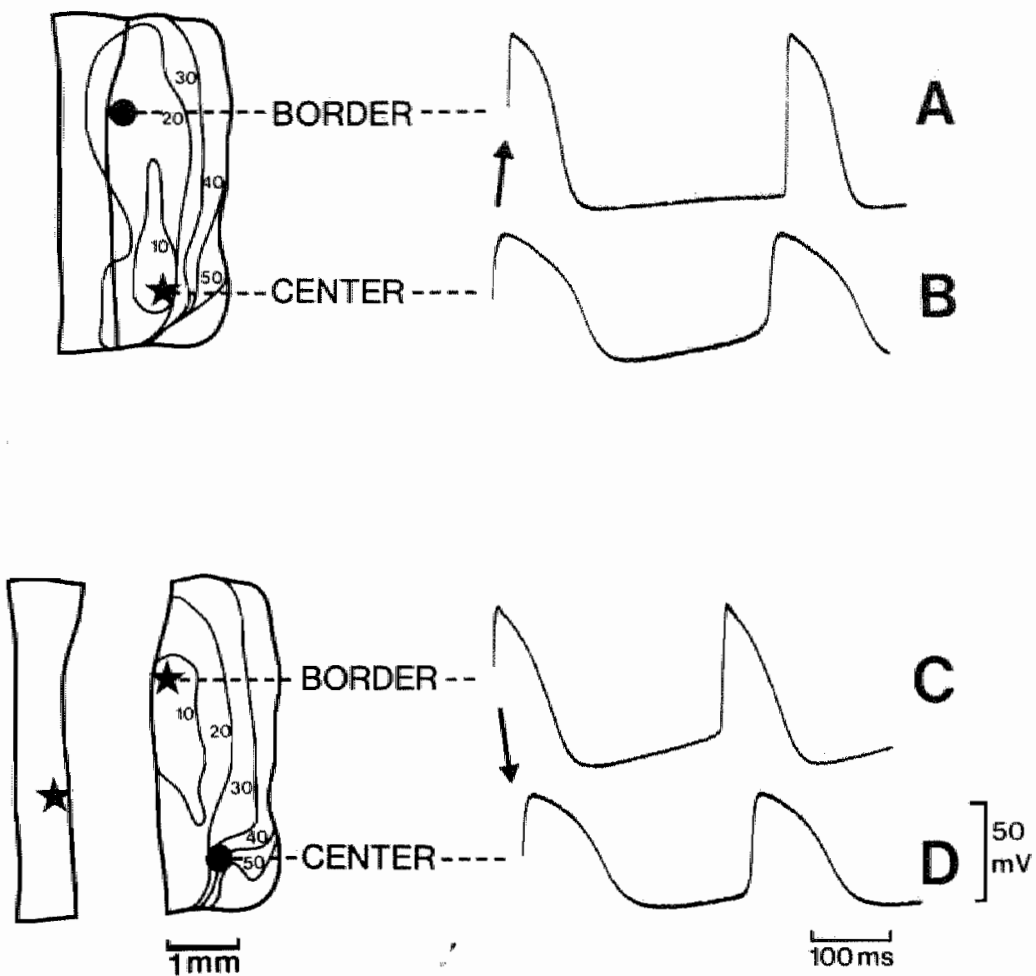

\section{Figure 2.2:}

The sequence of excltation in the sinus node, and action potentlal configuration in pacemaker sites before and after sino-atrial disconnection. The upper panel deplcts the control situation. The pacemaker was found in the center of the sinus node. From this point the impulse propagated through the border zone towards the crista terminalis. The actlon potentials at the site of Impulse origin (B) showed the steepest slope of spontaneous diastollc depolarization. In the border zone this slope was less steep. The lower panel shows the activation map of the sinus node after removal of the crista terminalis. The pacemaker was shifted to the border zone which caused a reversion of the nodal sequence of excitation. Spontaneous diastolic depolarization in the border zone was in. creased drastically, whereas in the center of the node a small decrease was observed.

The lower map in figure 2.2 shows the activation pattern in the sinus node after disconnection from the crista terminalis. The pacemaker (asterisk) was shifted from its original position in the center of the node towards a new location in the border zone where it remained during the further course of the experiment. As a result of this pacemaker shift the activation pattern in the sinus node was completely reversed. Actually, the sinus impulse propagated retrogradely from the border zone to- 
wards the center of the sinus node. In all preparations $(n=13)$ with the pacemaker located in the nodal center, separation of the crista terminalis and the sinus node was followed by a shift of the pacemaker towards the border zone. The pacemaker location in the border zone was not related to a preferential area. In some experiments it was found in the cranial part of the border zone, in others it was shifted in caudal direction towards the inferior caval vein.

In two preparations (dotted lines in figure 2.1) the pacemaker was located in the border zone during control. In one of these cases removal of the atrium induced a shift of the pacemaker to another area in the border zone and a shortening of the sinus cycle length with $15 \mathrm{~ms}$. In the other experiment no changes were found neither in pacemaker location nor in sinus rate.

\section{The influence of the atrial myocardium on action potential configuration in nodal fibers}

In the right part of figure 2.2 typical action potentials are shown as recorded from fibers in the border zone and center of the sinus node. The sites of recording correspond to the location of the pacemaker before (center) and after (border zone) sino-atrial disconnection. Under control conditions (upper panel) the action potentials of fibers in the center of the sinus node $(B)$ exhibited a configuration which was typical for leading pacemaker cells: a slow phase-0 depolarization, a long duration, a low amplitude and, finally, a steep spontaneous diastolic depolarization which was followed by a smooth transition to the next upstroke. In the border zone (A) the action potentials showed a steeper phase- 0 depolarization, a shorter duration, a higher amplitude and a lower rate of spontaneous diastolic depolarization, followed by a sudden transition to the upstroke of the next response. As expected, the fibers in the border zone were activated later than the fibers in the center of the sinus node. After removal of the atrial tissue mass however (lower panel), the rate of spontaneous diastolic depolarization was changed markedly. This is illustrated by the action potentials in the lower part of the figure. These recordings were obtained from the same locations as before (A and B). In the border zone fibers (C) the slope of diastolic depolarization had become much steeper, whereas the action potentials from fibers in the center of the sinus node showed a slight decrease in diastolic depolarization rate (D).

To quantify the changes in action potential configuration multiple intracellular recordings were made in 5 experiments. In each preparation two series of 10 recording sites were arranged in two lines running parallel to the crista terminalis. One recording line was located in the border zone at a distance of $0.3 \mathrm{~mm}$ from the sino- 


\section{Table 2.1}

Changes in Action Potential Configuration

$n=5$

CONTROL

DISCONNECTED sign. SINUS NODE

\section{BORDER ZONE}

Spontaneous Diastolic Dep. (mV/s)

$26 \pm 12$
$-61 \pm 6$
$59 \pm 9$
$74 \pm 13$

$78 \pm 16$

龂

Maximal Diastolic Potential (mV)

$-62 \pm 6$

n.s.

Action Potential Duration (ms)

Action Potential Amplitude (mv)

$74 \pm 13$

$67 \pm 9$

$67 \pm 10$

ste

*⿻一𠃋十

\section{CENTER}

Spontaneous Diastolic Dep. (mV/s)

$52 \pm 19$
$-56 \pm 11$
$67 \pm 19$
$56 \pm 12$

$50 \pm 28$

n.s.

Maximal Diastolic Potential (mV)

Action Potential Duration (ms)

Action Potential Amplitude (mV)

$56 \pm 12$

$-56 \pm 10$

n.s.

$72 \pm 11$

$51 \pm 13$

***

**

The mean values of different action potential parameters, determined from multiple intracellular registrations in the border zone and center of the shus node at 0.3 and $0.8 \mathrm{~mm}$ distance from the crls* ta terminalis respectively. Significance (paired) to Control: * $p<0.05_{*}^{*} *{ }^{*} p<0.01$; $* \pi * p<0.001$

atrial transition whereas the other series of impalements was made in the center of the sinus node at $0.8 \mathrm{~mm}$ from the atrium. The distance between the neighbouring impalements was $0.3 \mathrm{~mm}$. The action potential parameters were measured before and after removal of the atrium. The results are summarized in table 2.1. After sinoatrial disconnection the spontaneous diastolic depolarization rate in the border zone of the sinus node increased drastically. The action potential duration increased significantly as well, whereas the amplitude was reduced. In the center of the sinus node no significant change in the rate of spontaneous diastolic depolarization was found. The duration and amplitude of the action potentials changed to a similar amount as in the border zone. 

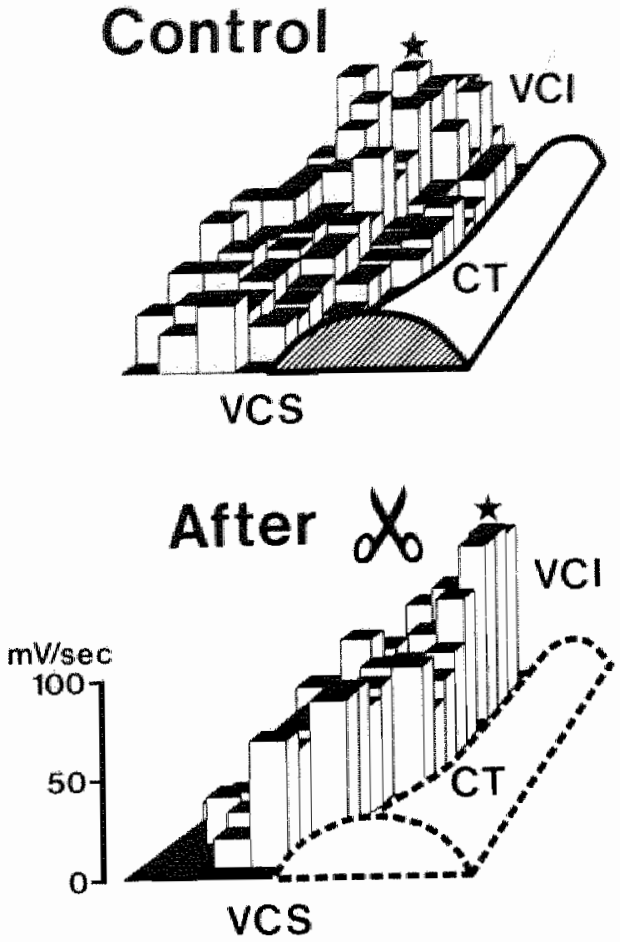

Figure 2.3:

Spatial distribution of rate of spontaneous diastolic depolarization in the sinus node before (upper panell) and after (lower panel) sino-atrial disconnection. Intracellular registrations were made with a spatial resolution of $0.3 \mathrm{~mm}$. The altitude of the vertical bars represents the steepness of spontaneous diastolic depolarization (mV/s) at the corresponding site. The asterisk indicates the site of eartiest electrical activaty. Compared to the activation maps in figure 2.2 the preparation is turned over 180 in its horizontal plane. In the lower panel the Imaginary contours of the crista terminalis are given (dotted line).

VCS = Vena Cava Superior; $\mathrm{VCl}=$ Vena Cava Inferior; $\mathrm{CT}=$ Crista terminalis .

\section{Spatial distribution of the spontaneous diastolic depolarization rate}

To study the spatial distribution of the slope of spontaneous diastolic depolarization, we measured this parameter in a large number $(80-100)$ of sites in the sinus node both before and after sino-atrial disconnection. The results of one of these experiments are shown in figure 2.3. The rate of spontaneous diastolic depolarization $(\mathrm{mV} / \mathrm{s})$ is represented by vertical bars, plotted in relation to the contours of the preparation. The upper panel shows the situation before, the lower panel after removal of the atrium. Under control conditions the highest rates of spontaneous diastolic depolarization were found in a distinct area, located in the caudal part of the sinus node at a distance of $1.2 \mathrm{~mm}$ from the crista terminalis. This area corresponded to the central portion of the sinus node. The site of steepest diastolic depolarization coincided with the site of earliest activation (asterisk). The lower panel shows the situation after removal of the atrium. As can be seen clearly, the rate of spontaneous 


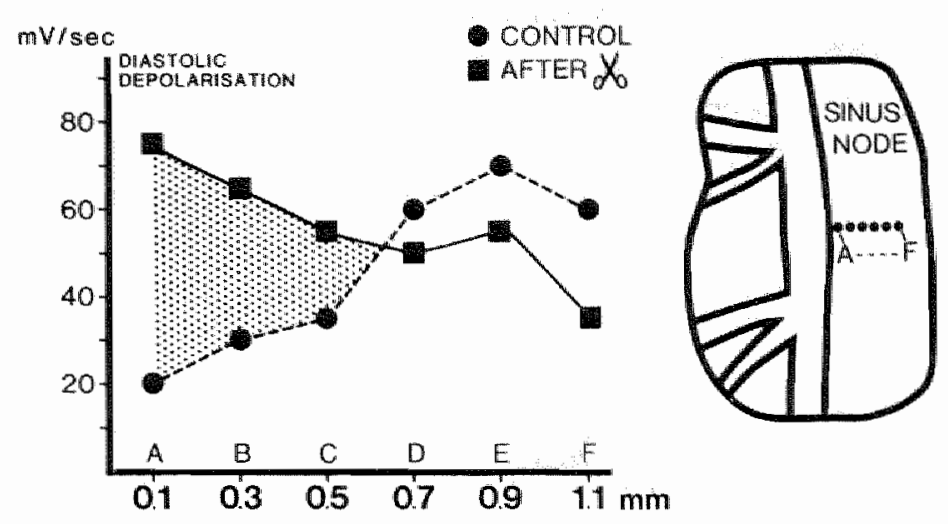

Figure 2.4:

Spontaneous diastolic depolarization rate (ordinate) as a function of the sino-atrial distance (abscissa). The scheme in the right shows the individual recording sites related to the contours of the preparation. The relationship between diastolic depolarization rate and sino-atrlal distance is reversed after separation of atrifum and sinus node. The shaded area represents the electrotonic depression of the fibers in the border zone by the attached atrial myocardium.

diastolic depolarization was increased in the entire border zone. In contrast to the center of the sinus node where no such increase was observed. The point of steepest spontaneous diastolic depolarization was migrated to the border zone and explained the pacemaker shift (asterisk) to this site after sino-atrial disconnection. Similar results were obtained in the other experiments in which the pacemaker was shifted to the border zone.

To determine whether the changes in the rate of spontaneous diastolic depolarization were related to the distance from the atrium, a number of intracellular recordings were made in the sinus node at increasing distances from the crista terminalis. The diagram in figure 2.4 shows the results obtained from one of these experiments. During control (filled circles) the diastolic depolarization rate at a sino-atrial distance of $0.1 \mathrm{~mm}$ was only $20 \mathrm{mV} / \mathrm{s}$. At greater distances from the atrium the rate gradually increased. The maximum rate of $70 \mathrm{mV} / \mathrm{s}$ was found in the center of the sinus node at a distance of $0.9 \mathrm{~mm}$ (site E). Apparently, under control conditions the rate of spontaneous diastolic depolarization gradually increased from the crista terminalis towards the center of the sinus node. However, after separation of atrium and sinus node (filled squares) this relationship was reversed. Now a maximal diastolic depolarization rate of $75 \mathrm{mV} / \mathrm{s}$ was found in site $A(0.1 \mathrm{~mm})$ and was even higher than the maximal value during control. At greater sino-atrial distances the rate of diastolic depolarization decreased gradually. In site $E(0.9 \mathrm{~mm})$ a rate of $57 \mathrm{mV} / \mathrm{s}$ was 
found. This was $13 \mathrm{mV} / \mathrm{s}$ less than under control conditions when the pacemaker was located in this area.

The increase in slope of spontaneous diastolic depolarization in the border zone (shaded area in the diagram) represents the depressive effect of the attached atrium. Similar results as shown in figure 2.4 were obtained in the other experiments.

\section{Discussion}

To study the influences of the atrium on impulse formation in the isolated sinus node it was necessary to disconnect both tissues with a minimal amount of damage. Therefore, a small pair of scissors was used in combination with a dissecting microscope to separate the crista terminalis from the border zone of the sinus node.

It appeared that sino-atrial disconnection caused a marked increase in the rate of spontaneous diastolic depolarization of fibers in the border zone. This resulted in a sinus acceleration and a pacemaker shift from its usual position in the center of the sinus node towards a new location in the sino-atrial border zone. Two possible explanations are available for these phenomena. First, the fibers in the border zone have been damaged by the separation technique, resulting in a higher rate of spontaneous diastolic depolarization and an increased rate of firing. Second, under normal conditions the spontaneous diastolic depolarization in border zone fibers is electrotonically depressed by the attached atrial muscle. As a consequence, removal of the crista terminalis eliminates this depressive influence and elucidates the intrinsic pacemaker properties of the fibers in the border zone.

With respect to the first explanation it is obvious that a number of fibers, located at both sides of the cut, have been damaged. Nevertheless, a causal relationship of this injury with the observed phenomena seems unlikely for several reasons. If in the present experiments the increased sinus rate after sino-atrial disconnection was due to accelerated autornaticity in damaged fibers, one would expect this sinus tachycardia to be a short-term phenomenon, disappearing as soon as the injured cells are dead or 'healed-over". This 'healing-over' process lasts maximally $15 \mathrm{~min}$ $[59,61,63]$, whereas in the present experiments the accelerated sinus rhythm maintained during at least 4 hours. Furthermore, the maximal diastolic membrane potential showed no significant reduction after sino-atrial disconnection. In case of major fiber damage a marked decrease of this parameter in border zone fibers would be expected.

Another important argument against injury-induced sinus acceleration is provided by the results of an earlier study [33]. In the same preparation as used in the present experiments similar techniques were applied to produce an incision through 
the sinus node, running parallel to the crista terminalis at a distance of $0.5 \mathrm{~mm}$. In this way the sino-atrial border zone with attached atrium was seperated from the center of the sinus node. In both parts spontaneous activity resumed at a rate similar to the sinus rate before transnodal division (control sinus interval: 361 t29 ms; central portion $348 \pm 32 \mathrm{~ms}$, border zone part $357 \pm 31 \mathrm{~ms}, \mathrm{n}=25$ ) [33]. Apparently, the cut did not induce accelerated rhythms in either part of the sinus node although the amount of tissue-damage must have been similar to that in the present experiments.

The second explanation for the present observations proposes the existence of electrotonic interactions between atrial fibers in the crista terminalis and pacemaker fibers in the sinus node. The difference in diastolic membrane potential between both fiber types will induce a passive current flow. During electrical diastole this current flow causes a hyperpolarization of the fibers in the sinus node which reduces the rate of spontaneous diastolic depolarization. On the other hand, this passive current flow will also cause a minimal reduction of the atrial membrane potential. This, however, is probably indetectable because of the enormous tissue mass of the crista terminalis.

The amount of passive current flow between fibers in the sinus node and the crista terminalis depends on the quality of intercellular electrical coupling as well as on the sino-atrial distance. The present findings suggest that the atrial electrotonic influence does depress spontaneous diastolic depolarization in the border zone fibers but does not reach the center of sinus node. This is in agreement to the results of other authors who measured the space constant in the isolated sinus node (Bonke, 1973: $0.5 \mathrm{~mm}$ [31]; Seyama, 1976: $0.8 \mathrm{~mm}$ [242]; Bukauskas et al., 1977: 0.2-0.6 mm [45]; Bleeker et al., 1982: $0.41-0.52 \mathrm{~mm}$ [24]). From recent studies it appeared that the distribution of intercellular connections or 'gap junctions' in the sinus node is inhomogeneous $[102,170]$. In the center of the sinus node the density of gap junctions was found to be less than in the border zone. As a result, the space constant decreases from the border zone towards the nodal center. This shortens the distance of electrotonic depression into the sinus node with an additional amount. The density of intercellular connections is reported to be higher in the cranial portion of the sinus node [170]. This suggests that the electrotonic influence of the crista terminalis extends further into the cranial part of the sinus node and explains the lower rate of spontaneous diastolic depolarization found in that area (figure 2.3).

The electrotonic influence of the atrial myocardium not only decreases the rate of spontaneous diastolic depolarization but also other action potential parameters of nodal cells. Removal of the atrium was followed by a reduction in amplitude and an increase in duration of nodal action potentials. This can be explained as follows. The amplitude of atrial action potentials exceeds the amplitude of action potentials 
in sinus node fibers. Since the top of the action potentials of both fiber types will occur almost simultaneousily during sinus rhythm, a systolic potential difference develops. This results in a passive current flow which increases the amplitude of nodal fibers. The same mechanism modulates the duration of nodal action potentials. Since the repolarization phase of atrial fibers starts earlier than in the nodal fibers, the transient potential difference shortens the duration of nodal action potentials. Removal of the crista terminalis eliminates these electrotonic interactions. The minor decrease of the slope of spontaneous diastolic depolarization in the center of the sinus node after sino-atrial disconnection may be explained by the slight overdrive of the central pacemaker fibers during the accelerated border zone riythm.

The present findings are in agreement to those of other investigators [145, 194 , 200]. In recent studies the isolated sinus node was divided in small pieces. It was found that specimen which originated from the sino-atrial border zone often demonstrated a higher intrinsic rate of automaticity than the specimen from the center of the sinus node.

Several environmental changes like hypothermia and electrolyte disturbances, or administration of cardiac drugs or autonomic transmitters exhibit chronotropic actions and affect the position of the pacemaker within the sinus node $[36,37,165,250$, 264]. In this context, alterations in electrotonic influence between atrial and nodal cells might play a significant role. Cardiac glycosides for example, reduce the amount of intercellular electrical coupling between cells $[62,283]$. At higher concentrations these drugs may cause sinus acceleration and intranodal pacemaker shifts towards the sino-atrial border zone $[250,260]$.

From the present experiments it is concluded that: (1) the intrinsic pacemaker properties of fibers in the border zone of the isolated rabbit sinus node are electrotonically depressed by the attached atrial myacardium; (2) the intrinsic pacemaker properties of transitional fibers are as good as or even better than the intrinsic pacemaker properties of fibers in the center of the sinus noide. 


\section{Chapter III}

\section{AN EASY AND DIRECT APPROACH TO INVESTIGATE CONDUCTION PROPERTIES OF THE RABBIT SINUS NODE}

d Cardiovasc Pharmacol 11:667-675, 1988 



\section{Introduction}

In the isolated right atrium of the rabbit heart spontaneous impulse formation usually takes place in the center of the sinus node. From this point the sinus impulse propagates through the perinodal region towards the crista terminalis and finally activates the atrial myocardium. The characteristics of this sino-atrial impulse conduction can only be studied by reconstruction of the sequence of excitation in the sinus node, a procedure which requires multiple intracellular registrations $[23,169225,242$, 249]. This is a very time-consuming technique and can only be applied if both the rate and position of sinus impulse formation remain stable.

In the present chapter we introduce an easier approach to investigate sino-atrial impulse conduction and refractoriness in the isolated rabbit sinus node. In this model an incision is made in the right atrial wall, perpendicular to the crista terminalis, to obtain a preparation which consists of two atrial parts connected only by a bridge of sinus node tissue. In this preparation transnodal conduction of stimulated atrial impulses can be studied without intracellular recording techniques. The method was validated by reconstruction of the conduction pathway through the sinus node. The basic conduction properties in the border zone and the center of the sinus node were studied. In addition, the effects of hypothermia, moderate hypoxia, acetylcholine and norepinephrine were examined. The results of these experiments are also described in the present, chapter.

\section{Methods}

\section{The preparation}

Young New Zealand rabbits of either sex weighing $1.5-2 \mathrm{~kg}$ were killed by cervical dislocation. After midsternal thoracotomy the heart was quickly excised and immersed in warmed oxygenated Tyrode solution. After isolation of the right atrium a preparation was made including the roof of the atrial appendage and the intercaval tissue in which the sinus node is embedded. The AV node and interatrial septum were removed. The final preparation was mounted on a silicon bar with the endocardial side facing upward, and transferred into a tissue-bath. The tissue-bath ( 35 ml) was perfused $(100 \mathrm{ml} / \mathrm{min})$ with Tyrode solution containing in $\mathrm{mM}: \mathrm{NaCl} 130, \mathrm{KCl}$ 5.6. $\mathrm{CaCl}_{2} 2.2, \mathrm{MgCl}_{2} \mathrm{O} .6, \mathrm{NaHCO}_{3} 24.2, \mathrm{Na}_{2} \mathrm{PO}_{4} 1.2$, glucose 11 and sucrose 13. The fluid was saturated with a mixture of $95 \% \mathrm{O}_{2}$ and $5 \% \mathrm{CO}_{2}$. The $\mathrm{pH}$ was 
$7.35 \pm 0.05$. The temperature was kept constant at $38 \pm 0.2^{\circ}$ Celsius until the effects of graded hypothermia were studied.

\section{Quantification of conduction properties in the sinus node}

To study the conduction properties in the sinus node an atrial incision perpendicular to the crista terminalis was made by means of a fine pair of scissors. In this way the atrial part of the preparation was divided in two halves, being onlly connected by the sinus node. A schematic drawing of the final preparation is shown in figure 3.1. One of the atrial halves was paced by means of a bipolar surface electrode which was connected to a programmable stimulator (Janssen Scientific Instruments). The stimulator delivered rectangular pulses of $1 \mathrm{~ms}$ duration. The intensity of the stimuli was adjusted to twice the diastolic threshold in case of regular pacing and four times the diastolic threshold when premature stimuli were applied. From the point of stimulation the impulse could activate the other atrial half only after passing through the sinus node. This transnodal conduction was monitored by two bipolar surface electrodes, positioned on top of the crista terminalis next to the inci-

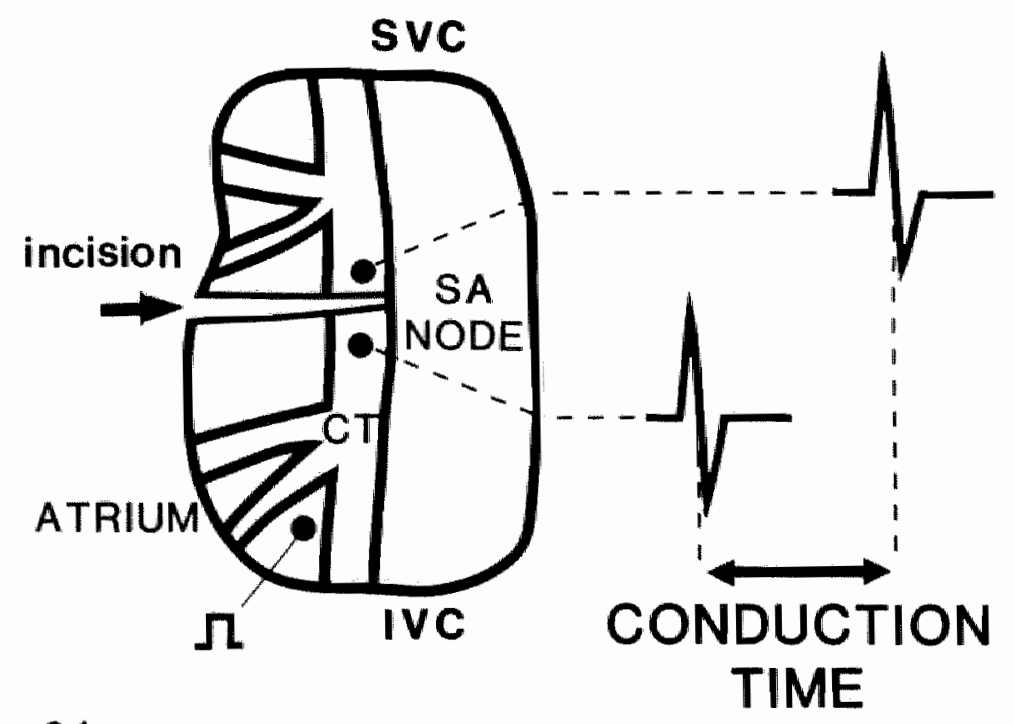

Figure 3.1:

Schematlc representation of the preparation used. An incislon was made through the atrium and the crista terminalis (CT). Two bipolar surface electrodes (black dots) were positioned on top of the crista terminalls at each side of the incision. From the recorded electrograms the transnodal conduction time could be measured during pacing at one of the atrial halves (square pulse at the lower lefti). $S V C=$ superior vena cava; IVC = inferlor vena cava; see text for further explanation. 
sion. In this way two atrial electrograms were recorded which represented the entrance and exit of the sinus node bridge (figure 3.1). The time interval between the electrical complexes in both electrograms represented the conduction time through the sinus node and was measured by a digital counter (Hewlett Packard). Both the recording and the stimulation electrodes consisted of teflon coated silver wire ( $0.25 \mathrm{~mm}$ diameter; $0.3 \mathrm{~mm}$ interpolar distance).

The length of the atrial incision into the nodal tissue determined the conduction route of the impulse through the sinus node. In preliminary experiments and on the guidance of intracellular recordings we determined the minimal length of the incision to obtain conduction either through the border zone or the center of the sinus node. It appeared that the impulse propagated through the border zone if the crista terminalis was completely transsected. This could be controlled by means of a dissecting microscope (magnification factor: $16 \mathrm{x}$ ). The impulse propagated through the center of the sinus node if the incision was extended by $0.6 \mathrm{~mm}$ into the nodal tissue sheet. These dimensions are in agreement to the results of morphological studies $[23,165]$.

\section{Experimental protocols}

In 4 experiments the spread of excitation in the sinus node was mapped during atrial pacing with an interval of $330 \mathrm{~ms}(3 \mathrm{~Hz})$. Multiple intracellular recordings were obtained by means of a single roving microelectrode (tip resistance 10-30 MOhm). The coordinates of the individual recording sites (about 70 in each experiment) were controlled by vernier scales and related to the anatomical landmarks of the preparation [237]. The cellular moment of activation was defined as the moment at which the upstroke of the action potential reached the $50 \%$ level of the amplitude. The steepest negative deflection in the proximal atrial electrogram served as time reference zero.

To quantify the conduction properties in the sinus node the following pacing protocol was executed: starting at a basic pacing rate of $3 \mathrm{~Hz}$ ( $330 \mathrm{~ms}$ interval), the pacing interval was shortened in steps of 5 ms until the shortest pacing interval (interval $F_{\text {max }}$ ) was reached whereby 1:1 transnodal conduction during at least 30 seconds was still present. The conduction time through the sinus node was measured at each pacing interval. Subsequently, single premature stimuli were applied to the atrium after every $15^{\text {th }}$ basic stimulus ( $330 \mathrm{~ms}$ interval). The conduction times of the premature beats were measured while shortening the coupling interval to the preceeding basic impulse in steps of $5 \mathrm{~ms}$ until the premature beat blocked in the sinus node. Finally, both the functional refractory period (shortest $A_{1}-A_{2}$ at the distal sur- 
face electrogram) and the effective refractory period (shortest $\mathrm{S}_{1}-\mathrm{S}_{2}$ still conducted) were measured with an accuracy of $1 \mathrm{~ms}$.

In a number of experiments we studied the effects of graded hypothermia, moderate hypoxia, and autonomic transmitters on conduction properties of the sinus node. The effects of hypothermia were examined by stepwise lowering of the temperature of the heat-exchange system. Within 5 minutes a new constant temperature was reached. To create moderate hypoxic conditions the Tyrode solution was saturated with a mixture of $95 \% \mathrm{~N}_{2}$ and $5 \% \mathrm{CO}_{2}$. Thus the $\mathrm{pO}_{2}$ in the tissuebath was reduced from about $400 \mathrm{mmHg}$ (normoxia) to $70-90 \mathrm{mmHg}$. Finally, in a series of experiments we studied the influences of acetylcholine $\left(13.8 \times 10^{-6} \mathrm{M}\right)$ and norepinephrine $\left(3 \times 10^{-6} \mathrm{M}\right)$ on conduction velocity and refractoriness in the sinus node. The given concentrations of both autonomic transmitters were chosen because of an equivalent chronotropic effect.

No measurements were performed within the first $60 \mathrm{~min}$ after producing the atrial incision. The Student-t-test (paired, two-tailed) was used for statistical analysis of the obtained data.

\section{Results}

\section{Validation of the method}

In 4 experiments we reconstructed the transnodal conduction pathway during slow atrial pacing ( $330 \mathrm{~ms}$ cycle length) by intracellular mapping. In each experiment activation maps were produced with an atrial incision just through the crista terminalis, and after extention to $0.4 \mathrm{~mm}$ and $1.0 \mathrm{~mm}$ into the sinus node. The results of one of these experiments are shown in figure 3.2. In this example the inferior atrial half was stimulated (pulse sign). The moment the impulse reached the proximal surface electrode was taken as time reference zero. The activation pattern in the sinus node is visualized by isochrones of $10 \mathrm{~ms}$. During atrial pacing the impulse travelled invarlably along the shortest pathway, Just around the tip of the incision, towards the distal atrial part of the preparation. With an incision just through the crista terminalis (left panel) the impulse propagated through the sino-atrial border zone. When the cut was extended to 0.4 (middle panel) or to $1.0 \mathrm{~mm}$ (right panel) into the sinus node, the impulse propagated through more central regions of the sinus node. In these areas the conduction velocity around the tip of the incision was markedly slower (narrowing of the isochrones). As a result, the transnodal conduction time increased from 26 to 46 and $86 \mathrm{~ms}$ respectively. In the right map the impulse travelled clearly through the center of the sinus node. The activation maps demon- 

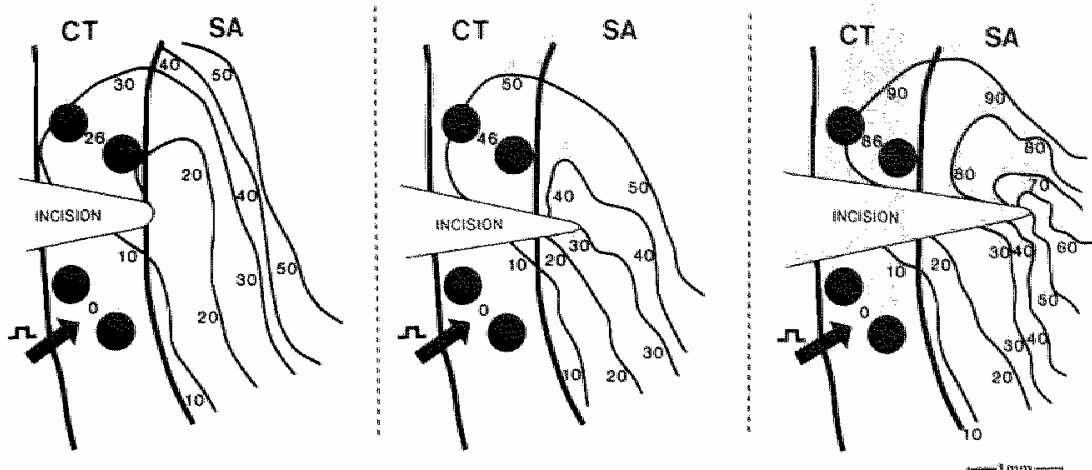

\section{Figure 3.2:}

The activation pattern in the crista terminalls and sinus node during atrlal pacing with a cycle length of $330 \mathrm{~ms}$. The sequence of excltation was mapped by means of a single roving microelectrode (about 70 impalements, $0.2-0.4 \mathrm{~mm}$ spatial resolution). Isochrones of $10 \mathrm{~ms}$ are drawn to visuallze the spread of excitation. Incisions just to the border zone (left panel), $0.4 \mathrm{~mm}$ (mlddle panel) and $1.0 \mathrm{~mm}$ ( $r$ 'ght panel) into the sinus node were made. As can be seen, the stimulated impulse always propagated along the shortest pathway, i.e. Just around the tip of the incision, to the olher atrial part. $\mathrm{CT}=$ crista terminalis; $\mathrm{SA}=$ sinus node.

strate that the length of the incision into the sinus node determined the nodal region where the impulse was forced to pass through.

In figures 3.3 and 3.4 the rate-dependency of impulse conduction in the sinus node is illustrated. On both sides of an incision into the nodal center $(0.6 \mathrm{~mm})$, a microelectrode was impaled at a distance of $0.3 \mathrm{~mm}$ from the crista terminalis. The superior atrial part was stimulated. In both panels of figure 3.3 these simultaneously recorded intracellular signals are plotted together with the atrial electrograms from the crista terminalis. During atrial pacing with an interval of $310 \mathrm{~ms}$ (upper panel) the conduction time between the atrial surface electrodes was $49 \mathrm{~ms}$. Shortening of the pacing interval to $250 \mathrm{~ms}$ (lower panel) prolonged this conduction time to $65 \mathrm{~ms}$, being a rate-dependent increase of $16 \mathrm{~ms}$. The increase in conduction time between the upstrokes of the action potentials was $15 \mathrm{~ms}$ (from 21 to $36 \mathrm{~ms}$ ). This indicates that the rate-dependent lengthening of the transnodal conduction time was mainly caused by deceleration of the impulse around the tip of the incision. In figure 3.4 a number of five successive cycles are superimposed to illustrate the occurrence of Wenckebach phenomena. Atrial pacing with a cycle length of $215 \mathrm{~ms}$ resulted in a 5:4 coupling between the proximal and distal electrograms. The proximal (upper) intracellular recording showed a fixed time delay between the upstroke of the action potentials and the deflection in the proximal atrial electrogram. The distal intracellu- 


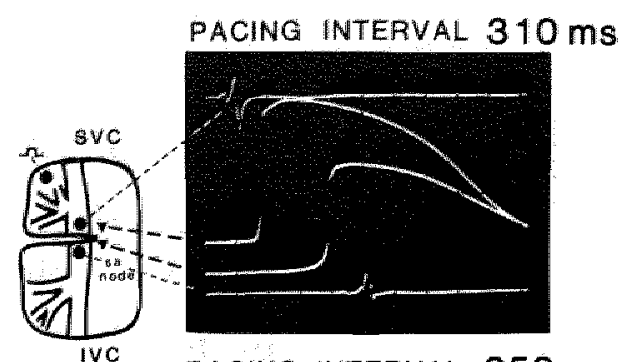

PACING INTERVAL $250 \mathrm{~ms}$

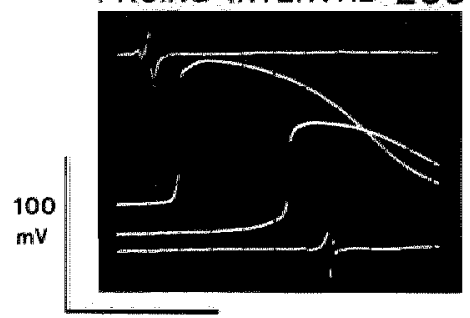

$50 \mathrm{~ms}$

\section{Figure 3.3:}

Rate-dependency of conduction through the sinus node. Two microelectrodes were simultaneously impaled at either side of the tip of an incision into the center of the sinus node. The upper and lower tracings in each panel represent the atrial surface electrograms whereas the middle tracings show the intracellular recordings. The increase in conductlion time after shortening of the pacing interval from 310 to $250 \mathrm{~ms}$ occurs between the intracellullar recordings and thus in the tissue around the tip of the inclision.

\section{WENCKEBACH PHENOMENON}

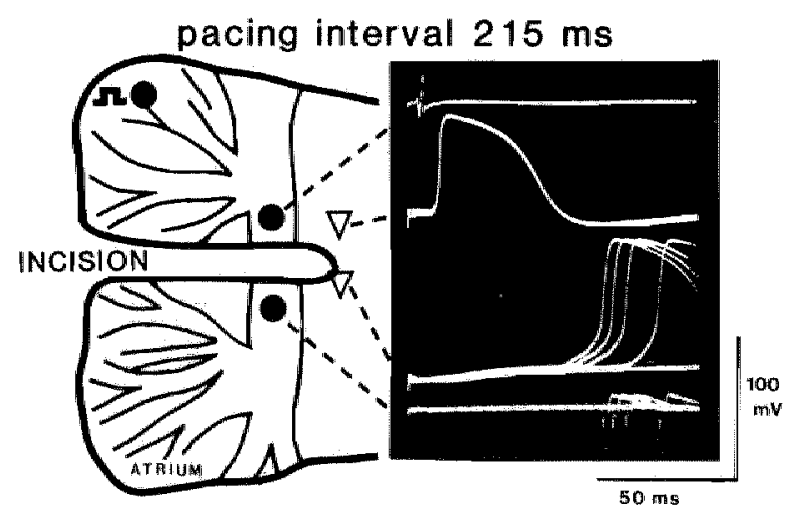

Figure 3.4:

'Wenckebach-conduction' (5:4) in the center of the sinus node during atrial pacing with an interval of 215 ms. The surface electrograms and intracellular recordings as described in flgure 3.3 are superimposed. As can be seen, a $5: 4$ conduction pattern developed in the tissue around the tip of the incislon.

lar recording, on the other hand, demonstrated a progressively increasing time delay to the proximal intracellular response. Finally, the fifth impulse blocked somewhere around the tip of the incision which is illustrated by the equipotential sweep in the distal tracing. These findings demonstrate that the area of the sinus node which is located around the tip of the incision determines the rate-dependent characteristics of transnodal impulse conduction in the present model.

In the present experiments also the influence of the direction of impulse conduction was considered. It appeared that the conduction time through the sinus node 
showed a maximal variation of $1 \mathrm{~ms}$ when switching the site of stimulation from the upper to the lower atrial part or reversed. All conduction parameters remained stable during at least six hours. Only a minimal increase in spontaneous sinus interval was found of about $10 \mathrm{~ms}$ per hour. This was also found in experiments in which no incision was made, and was described by others who used the isolated rabbit sinus node [165].

\section{Basic conduction properties in the border zone and center of the sinus node}

During incremental atrial pacing the conduction time through the sinus node increased progressively until the interval Fmax was attained. In figure 3.5 the transnodal conduction time is plotted as a function of the atrial pacing interval for three incisions of increasing length (see figure 3.2). The degree of rate-dependent prolongation of conduction time was least prominent with an incision just to the border zone of the sinus node (lower curve). In the present example conduction time during slow pacing was $20 \mathrm{~ms}$ and showed only a limited rate-dependent increase at relatively short pacing intervals. At the shortest pacing interval of $170 \mathrm{~ms}$ the conduction time was prolonged to $25 \mathrm{~ms}$. When the incision was extended into the sinus

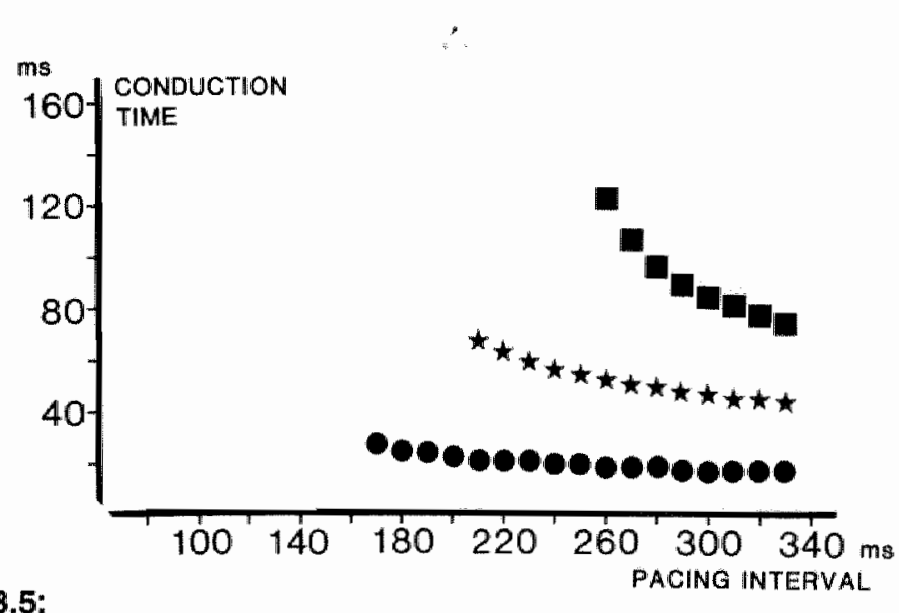

Figure 3.5:

Dlagram showing the transnodal conduction time as function of the atrlal pacing interval in different areas of the sinus node. The incision was made Just through the crista terminalls (fitled circles), $0.4 \mathrm{~mm}$ deeper (asterisks) and finally another $0.6 \mathrm{~mm}$ deeper (filled squares). During incremental atrial pacing, starting at an interval slightly shorter than the spontaneous sinus cycle length and ending at the interval $F_{\text {max }}$, the conduction time showed a progressive rate-dependent increase which was most prominent in the center of the sinus node (upper curve). 
node, the degree of rate-dependency increased markedly. In case of conduction through the center of the sinus node (upper curve) the conduction time during slow pacing ( $330 \mathrm{~ms}$ interval) was $80 \mathrm{~ms}$ and prolonged to $123 \mathrm{~ms}$ when the shortest pacing interval of $260 \mathrm{~ms}$ was reached. Similar relationships were found between the coupling interval and transnodal conduction time of premature impulses. The only difference was that premature impulses delived during slow pacing could be conducted with a higher degree of prematurity and a lower conduction velocity than a train of rapidly succeeding impulses.

In a large number of experiments we measured the conduction parameters in the border zone and center of the sinus node. In case of conduction through the nodal center the standard incision length of $0.6 \mathrm{~mm}$ was considered (see "methods" section). The results of these experiments are summarized in table 3.1. In a number $(n=12)$ of preparations the length of the conduction route through the sinus node was estimated by means of a microscope. Thus we could calculate that during slow pacing $(3 \mathrm{~Hz})$ the average conduction velocity in the sino-atrial border zone lies in between 7 and $11 \mathrm{~cm} / \mathrm{s}$. In the center of the sinus node the average conduction velocity was estimated between 2 and $5 \mathrm{~cm} / \mathrm{s}$.

\section{Table 3.1}

Conduction Properties In The Isolated Rabbit Sinus Node

$\begin{array}{cc}\text { BORDER ZONE } & \text { CENTER } \\ (n=59) & (n=39)\end{array}$

$\begin{array}{lrlrrr}\text { Minimal Pacing Interval (Fmax) } & 157 & \pm 2 & 240 & \pm 38 & \mathrm{~ms} \\ \text { Effective Refractory Period* } & 105 & \pm 16 & 165 & \pm 29 & \mathrm{~ms} \\ \text { Functional Refractory Period* } & 129 \pm 21 & 217 & \pm 42 & \mathrm{~ms} \\ \text { Conduction Time at } 3 \mathrm{~Hz} \text { pacing* } & 18 \pm 6 & 42 & \pm 24 & \mathrm{~ms} \\ \text { Conduction Time at Fmax } & 34 \pm 12 & 73 & \pm 37 & \mathrm{~ms} \\ \begin{array}{c}\text { Conduction Time of the Earliest } \\ \quad \text { Premature Beat* }\end{array} & 51 \pm 26 & 125 & \pm 61 & \mathrm{~ms}\end{array}$

* In a limited number of experiments spontaneous sinus interval was shorter than $330 \mathrm{~ms}(3 \mathrm{~Hz}$ ). Therefore the preparation was paced with a baslc interval of $300 \mathrm{~ms}$. All values are given as mean $\pm S D$. 


\section{The effects of hypothermia}

In 5 experiments the effects of graded hypothermia on impulse conduction through the sinus node were studied. Periads ( $45 \mathrm{~min}$ ) of normothermia $\left(38^{\circ} \mathrm{Cel}-\right.$ sius) were alternated with equally long episodes of hypothermia $\left(35.5^{\circ}, 33^{\circ}\right.$ or $30.5^{\circ}$ Celsius). In figure 3.6 the results of one of these experiments are depicted. The diagram shows the rate-dependency of conduction time through the border zone of the sinus node during the tested temperatures.

Lowering the temperature of the superfusate from 38 to $35.5^{\circ}$ Celsius reduced the maximal pacing rate (shift of the curve to the right) and increased the transnodal conduction time at fixed pacing intervals. This effect was doubled with a temperature of $33^{\circ}$ Celsius. After lowering the temperature to $30.5^{\circ}$ Celsius a marked prolongation of both the interval $F_{\max }$ and the conduction time was found.

In table 3.2 the normalized effects of increasing degrees of hypothermia are summarized. Conduction properties in the border zone and in the center of the sinus node were equally depressed. In the present experiments hypothermia exerted no irreversible influences on conduction properties in the sinus node. The conduction velocity and refractory period during the four intermittent stages of normothermia varied less than $1 \%$.

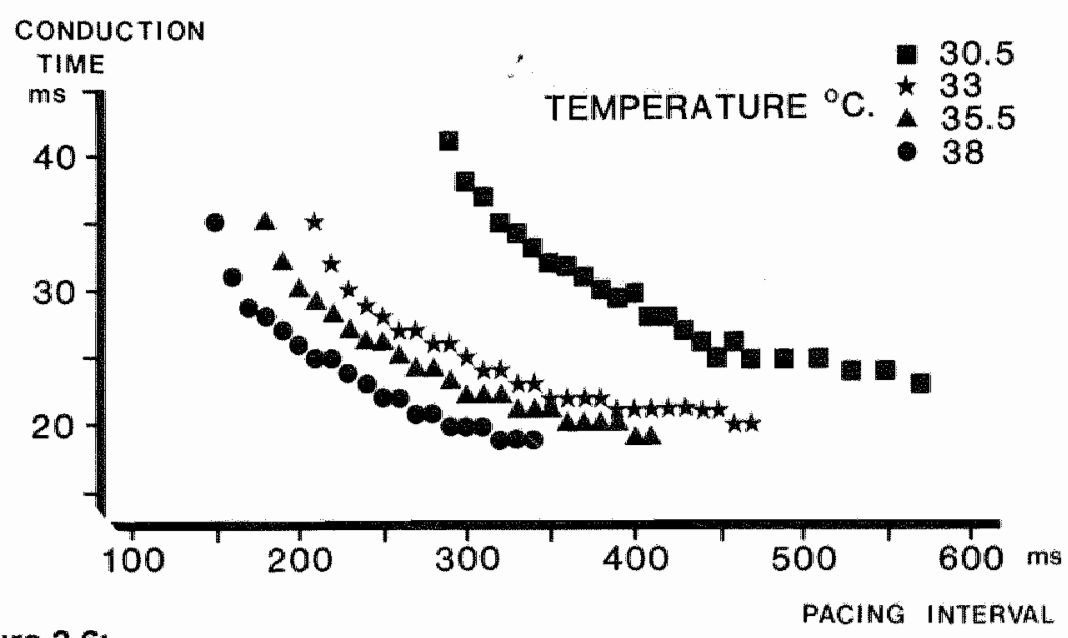

Figure 3.6:

Conduction through the border zone of the sinus node at different temperatures. The conduction time and interval $F_{\max }$ were prolonged as a result of increasing degrees of hypothermla (shift of the curves to the rlght). During normothermla ( $\left.38^{\circ} \mathrm{C}\right)$ the conductlon time during extremely low pacing rates could not be measured because of a hilgher sinus rate. 
Table 3.2

Effects Of Hypothermia On Sinus Node Conduction Properties

$n=5$

Sinus Rate

Conduction Velocity at $3 \mathrm{~Hz}$ pacing

Minimal Pacing Interval ( $F_{\text {max }}$ )

Effective Refractory Period $35.5^{\circ}$

$-16 \%$

$-6 \%$

$+16 \%$

$+25 \%$ $33.0^{\circ}$

$-28 \%$

$-14 \%$

$+33 \%$

$+56 \%$ $30.5^{\circ} \mathrm{C}$

$-40 \%$

$-35 \%$

$+75 \%$

$+92 \%$

All data were normallzed to the contral values ( $38^{\circ}$ Celsius).

\section{The effects of moderate hypoxia}

In 6 experiments we studied the effects of moderate hypoxia (90 min) on impulse conduction and refractoriness in the border zone and center of the sinus node. The results of one of these experiments are shown in figure 3.7. In the border zone (upper panel) an increase in conduction time and a slight prolongation of the minimal pacing interval was observed. Conduction through the center of the sinus node however (lower panel), was affected drastically. 90 minutes of moderate hypoxia

\section{Table 3.3}

Effects Of Hypoxia On Sinus Node Conduction Properties

$\begin{array}{cc}\text { BORDER ZONE } & \text { CENTER } \\ (\mathrm{n}=3) & (\mathrm{n}=3)\end{array}$

Sinus Rate

Conduction Velocity at $3 \mathrm{~Hz}$ pacing

Minimal Pacing Interval $\left(F_{\max }\right)$

Effective Refractory Period
$-29 \%$

$-24 \%$

$+18 \%$

$+1 \%$
$-29 \%$

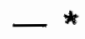

$+54 \%$

$+58 \%$

All date were normalized to the control values.

*During hypoxia the flbers in the center at the sinus node could not be paced with $3 \mathrm{~Hz}$. 

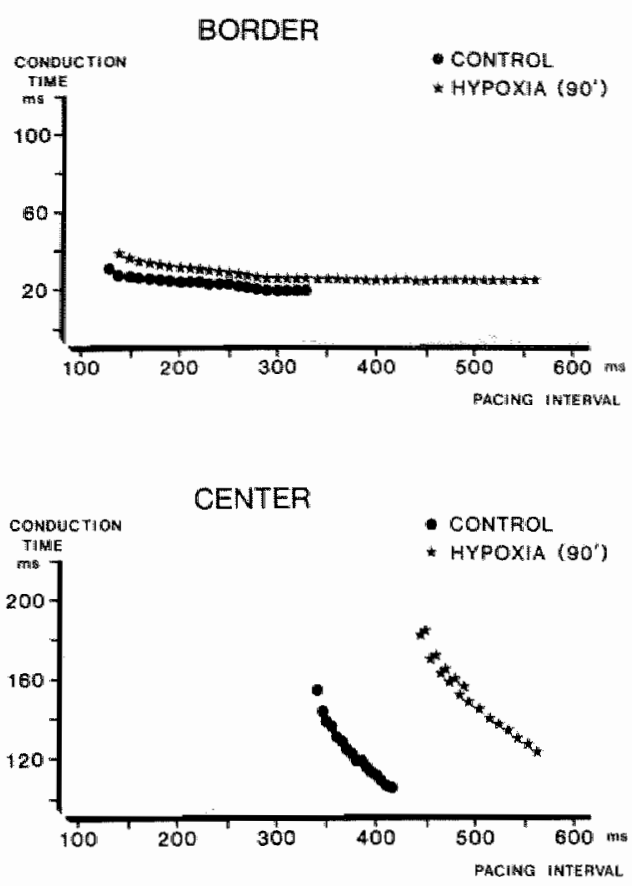

\section{Figure 3.7:}

The effects of $90 \mathrm{~m} / \mathrm{n}$ moderate hypoxia on $1 \mathrm{~m}$ pulse conduction through the border zone (upper diagram) and center of the sinus node flower dlagram). In the border zone only a limited change in interval $F_{\text {max }}$ but an evident prolongation in conduction time appeared. In the center of the sinus node a very strong effect on the Interval $F_{\text {max }}$ and transnodal conduction time was found. In the center conductlon time during pacing at $330 \mathrm{~ms}$ cycle length $(3 \mathrm{~Hz})$ could not be: compared, but the effect is evident.

caused a shift of the curve in up-and rightward direction indicating an increase in conduction time and a prolongation of the minimal pacing interval. In this example the latter parameter increased from 340 to $445 \mathrm{~ms}$. The present degree of hypoxia caused a marked reduction in sinus rate. As a consequence, conduction through the sinus node could be studied at far lower pacing rates than during normoxia. In table 3.3 the percentual effects of hypoxia on the conduction properties in the border zone and center of the sinus node are summarized. Obviously, conduction properties in the sino-atrial border zone were much less depressed than in the nodal center. In the present experiments the effects of hypoxia were completely reversible within 1 hour of normoxia.

\section{Acetylcholine and norepinephrine}

In 12 experiments we investigated the effects of acetylcholline $\left(13.8 \times 10^{-6} \mathrm{M}\right)$ and norepinephrine $\left(3 \times 10^{-6} \mathrm{M}\right)$ on sinus node conduction properties. In 6 of these experiments the effects on conduction velocity and refractory period in the crista terminalis were investigated as well. Figure 3.8 shows a typical example of the effects 
of acetylcholine and norepinephrine in the center of the sinus node during incremental atrial pacing. Acetylcholine shifted the curve to the right and caused a marked prolongation of the interval $F_{\max }$. As a result of this rightward shift, the conduction time at a given pacing rate was increased. Norepinephrine exhibited an opposite effect and shifted the curve leftward. This resulted in a shortening of the interval $F_{\max }$ and a decrease of the transnodal conduction time. The results of all experiments are summarized in table 3.4. Acetylcholine caused a significant shortening of the interval $F_{\text {max }}$ and the effective refractory period in the crista terminalis. During slow pacing the conduction velocity in this major atrial bundle was hardly affected. In the center of the sinus node acetylcholine caused a marked increase in transnodal conduction time during slow pacing as well as a significant prolongation of the effective refractory period and interval $F_{\max }$. Apparently, acetylcholine affected refractoriness in the atrium and center of the sinus node in an opposite direction. The refractory period in the border zone however, was not significantly altered by acetylcholine and seemed to react intermediate between atrial fibers and central nodal pacemaker fibers. Norepinephrine did not affect refractoriness in the atrium and caused only a limited shortening of this parameter in the border zone. In the center of the sinus node however, a marked decrease of the refractory period was found after addition of norepinephrine.

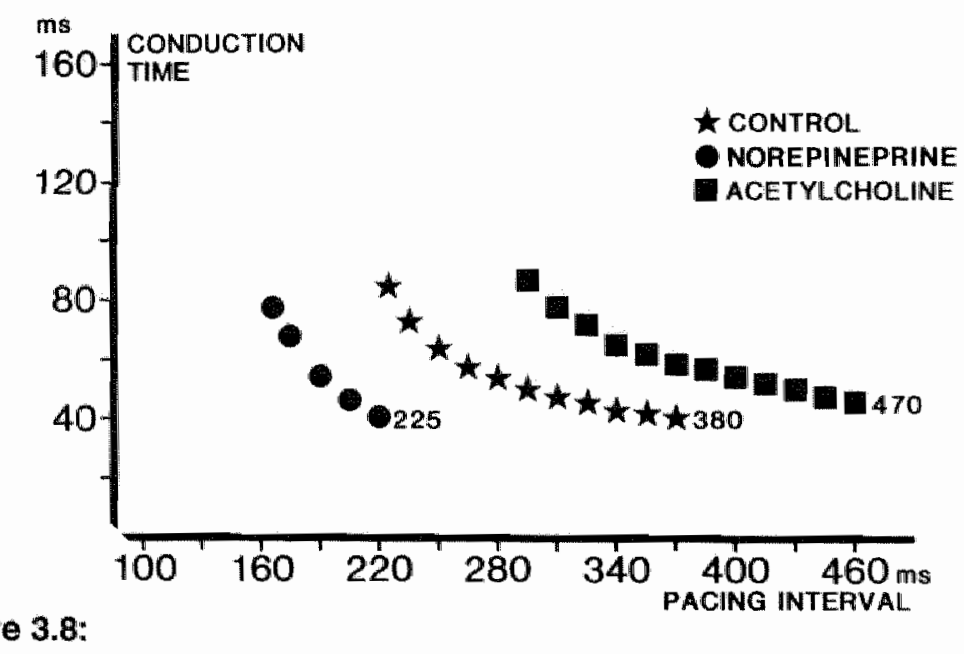

Figure 3.8:

The effects of autonomic transmitters on conduction through the center of the sinus node. The interval $F_{\text {max }}$ was shortened by norepinephrine and prolonged by acetylcholine. Conduction time was increased by acetylcholine and shartened by norepinephrine. Spontaneous sinus interval (ms) is given at the right tall of each curve. 
Tabje 3.4

The Effects Of Acetylcholine And Norepinephrine On Conduction Properties of The Sinus Node And Atrial Myocardium.

CONTROL ACH NE

$\left(13.8 \times 10^{-6} M\right) \quad\left(3 \times 10^{-6} M\right)$

CRISTA TERMINALIS $(n=6)$

Conduction Time at $3 \mathrm{~Hz}$ pacing Minimal Pacing Interval $\left(F_{\max }\right)$

Effective Refractory Period

$$
\begin{array}{rrrr}
5 & \pm 2 & 5 & \pm 2 \\
99 & \pm 19 & 79 \pm 17^{*} & 101 \pm 17 \\
62 & \pm 7 & 49 \pm 6^{* *} & 67 \pm 14
\end{array}
$$

\section{BORDER ZONE $(n=12)$}

Conduction Time at $3 \mathrm{~Hz}$ pacing

$19 \pm 6$

$21 \pm 7$

Minimal Pacing Interval (Fmax)

$131 \pm 17$

$135 \pm 38$

$123 \pm 18$

Effective Refractory Period

Functional Refractory Period

$93 \pm 12$

$87 \pm 16$

$91 \pm 10$

Spontaneous Sinus Interval

$114 \pm 11$

$105 \pm 17^{*}$

$107 \pm 13^{*}$

$366 \pm 44$

$440 \pm 39^{* * *}$

$229 \pm 23^{\text {*** }}$

\section{CENTER $(n=12)$}

Conduction Time at $3 \mathrm{~Hz}$ pacing

$51 \pm 18$

$76 \pm 45^{*}$

Minimal Pacing Interval ( $\left.F_{\max }\right)$

$238 \pm 48$

$303 \pm 59^{\text {* }}$

$188 \pm 17^{\text {**** }}$

Effective Refractory Period

$157 \pm 32$

$244 \pm 65^{* * *}$

$142 \pm 28$

Functional Refractory Period

$228 \pm 51$

$302 \pm 80^{* *}$

$180 \pm 26^{* *}$

Spontaneous Sinus Interval

$350 \pm 55$

$453 \pm 43^{* * *} 229 \pm 23^{* \star *}$

Values are given in $\mathrm{ms}$ as mean $\pm S D_{i}$ Slgnfflcance (palred) to Control: * $\mathrm{p}<0.05$; $^{\text {* }} \mathrm{p}<0.01$; $* * * \mathrm{p}<0.001$

$\mathrm{ACH}=$ Acetylchollne; $\mathrm{NE}=$ Norepinephrine. 


\section{Discussion}

In the present experiments we studied the basic characteristics of impulse conduction and refractoriness in the isolated rabbit sinus node. The effects of rate and thythm, hypothermia, moderate hypoxia, and autonomic transmitters were assessed. if we combine the results of the present experiments with the findings of Smeets et al. [247] who studied conduction properties in the isolated left atrium of the rabbit, it becomes evident that the conduction velocity decreases and the refractory period lengthens when going from the atrium towards the center of the sinus node. In this context the following facts are relevant for the rabbit heart:

- the average conduction velocity in the atrium is about $60 \mathrm{~cm} / \mathrm{s}$, in the sino-atrial border zone 7 to $11 \mathrm{~cm} / \mathrm{s}$ and in the center of the sinus node 2 to $5 \mathrm{~cm} / \mathrm{s}$;

- the average effective refractory period of atrial fibers is $70 \mathrm{~ms}$, of border zone fibers $105 \mathrm{~ms}$ and of fibers in the center of the sinus node $165 \mathrm{~ms}$;

- the average maximal activation rate of atrial fibers is $11.8 \mathrm{~Hz}$, of border zone fibers $6.4 \mathrm{~Hz}$, and of central nodal fibers $4.2 \mathrm{~Hz}$;

- one single impulse can be conducted through the sinus node with a higher degree of prematurity and a lower conduction velocity than a train of rapidly succeeding impulses.

The present results are in agreement with the findings of other authors $[134,211$, 251].

A combination of gradually changing membrane kinetics and a an increasing amount of intercellular coupling resistance is probably responsible for the gradual depresslon of conduction properties in the rabbit sinus node. Conduction velocity in cardiac fibers primarily depends on the rate of phase-0 depolarization. In atrial fibers the steepness of this depolarization is high because of the contribution of the fast sodium inward current. In the center of the sinus node however, the rate of phase- 0 depolarization is much lower and solely based on the slow calcium inward current. On the other hand, impulse propagation also depends on the amount of intercellular coupling resistance which is much higher in the center of the sinus node than in the atrial myocardium. As a result of these differences, impulse conduction velocity in the sinus node is remarkably lower than in the atrium $[31,45,242]$. The refractory period in cardiac muscle fibers is primarily determined by the action potential duration. The pacemaker fibers in the center of the sinus node show a consider- 
ably longer action potential duration than the atrial fibers. Furthermore, also the recovery of excitability after depolarization may show a certain degree of time-dependency which markedly exceeds the duration of the action potential. Strauss and Bigger [253] found that the recovery of the maximal rate of phase-0 depolarization in perinodal fibers was characterized by a much higher degree of time-dependency than in atrial fibers.

The results of the present experiments indicate that the conduction properties in the sino-atrial border zone are intermediate between the conduction properties of atrial fibers and pacemaker fibers in the center of the sinus node. This was already suggested by Strauss and Bigger [253]. The fact that conduction velocity in the border zone is higher and the refractory period shorter than in the center of the sinus node is in agreement with the intermediate action potential characteristics of perinodal fibers. The phase- 0 depolarization in these fibers is based on a combination of the fast sodium and slow calcium inward currents $[142,144,145,196,292]$. The action potential duration in the border zone fibers exceeds the duration in atrial fibers but is shorter than in central pacemaker fibers. Furthermore, intercellular coupling resistance in the border zone is probably less than in the center of the sinus node but still higher than in the atrium as suggested by the findings of Masson-Pevet et al. [169]. As a consequence, the effects of certain interventions on impulse conduction through the border zone might be different from the effects in the atrium and center of the sinus node.

In the present experiments hypothermia influenced conduction properties in the border zone and the center of the sinus node to an equal extent. During slow pacing the transnodal conduction velocity decreased by $5 \%$ whereas refractoriness was prolonged by $10 \%$ with each degree Celsius temperature lowering. These effects were already suggested by Bouman et al. [37] who found a decrease in the rate of phase- 0 depolarization and an increase in action potential duration in the sinus node during hypothermia. Smeets et al. [247] studied the influence of hypothermia in the left atrium of the rabbit and observed a decrease in conduction velocity by about $3 \%$ as well as an increase in refractoriness of almost $9 \%$ per degree temperature lowering. These findings suggest an equivalent depressive action of hypothermia on conduction properties in the sinus node and atrial myocardium.

Senges et al. [240] showed that retrograde sino-atrial conduction decelerated markedly during hypoxia. This was confirmed by the results of the present experiments which revealed that the conduction velocity in the sinus node decreased under moderate hypoxic conditions. However, this negative dromotropic action was less prominent in the border zone than in the nodal center. With respect to refractoriness we found that both the interval $F_{\max }$ and the effective refractory period in the border zone were hardly affected by moderate hypoxia. This was in contrast to 
the center of the sinus node in which a marked prolongation of both parameters occurred. Nishi et al. [193] studied action potential configuration and ultrastructure of the fibers in the sinus node during hypoxia and described a similar difference in sensitivity between the border zone and center of the sinus node.

Acetylcholine and norepinephrine exhibited different effects on atrial and sinus node conduction properties. It appeared that acetylcholine hardly affected conduction velocity in the atrium and border zone of the sinus node. This is in agreement to the findings of others authors $[211,247]$. Conduction velocity in the center of the sinus node on the other hand, was depressed markedly. This predominant action of acetylcholine on fibers in the center of the sinus node might be explained by a depression of the slow inward current [263]. Acetylcholine shortened, as expected, the refractory period in the atrial myocardium, but exhibited an opposite effect on refractoriness in the center of the sinus node. In the sino-atrial border zone the effective refractory period and interval $F_{\max }$ were hardly influenced. This does not mean that the fibers in the border zone of the sinus node are insensitive to the actions of acetylcholine. The minor cholinergic effect on these fibers might be the net result of the opposite actions in the atrium and center of the sinus node. This illustrates the intermediate characteristics of the sino-atrial border zone. The effects of norepinephrine on refractoriness in the sinus node are limited. In the present experiments norepinephrine did not significantly alter the effective refractory period, neither in the border zone nor in the center of the sinus node. Only the interval $F_{\max }$ in the center was shortened.

The results of the present study demonstrate that the rabbit sinus node is a heterogeneous structure, not only in morphology and pacemaker properties but also with respect to conduction properties [237]. The present model allows the investigator to obtain data which could not be collected by using microelectrode techniques. Conduction properties can be studied independently of sinus automaticity, which is an important limitation of the indirect techniques to measure the SACT [191,254]. Furthermore, investigation of conduction properties in the sinus node can be combined easily with the evaluation of conduction properties in the atrium or crista terminalis. Since the isolated rabbit sinus node preparation remains stable for many hours during superfusion in a tissue-bath, it is possible to evaluate the effects of environmental changes and other interventions. Comparative studies on the effects of drugs on sinus function represent another important application of the present model. Doseresponse relationships can be obtained in a direct and reproducible manner. In the next two chapters the effects of a number of drugs on sinus function are studied by means of the present technique. 


\section{Chapter IV}

\section{EFFECTS OF BEPRIDIL ON CONDUCTION PROPERTIES \\ OF THE ISOLATED RABBIT SINUS NODE AND ATRIAL MYOCARDIUM}

J Cardiovasc Pharmacol 13:131-137, 1989 


\section{Introduction}

Bepridil hydrochloride (CERM) is used as an anti-anginal drug because of its vasodilating and oxygen-consumption reducing influences. In addition to these actions, bepridil also exhibits a wide scale of electrophysiological effects [9,52]. In experimental and clinical studies both a reduction in heart rate and a prolongation of the AV nodal conduction time and refractory period were frequently described $[17,34$, $47,52,68,79,90,125,126,129,131,139,221,229,245,277]$. These electrophysiological effects fit into the profile of actions of class IV antiarrhythmic drugs [275]. However, it was also found that bepridil reduces the steepness of the action potential upstroke in atrial, ventricular and purkinje fibers, and prolongs the refractory period in the atrial and ventricular myocardium. These effects correspond to the actions of quinidine and other class I antiarrhythmic drugs $[47,68,79,129,221,229,275]$. Regarding these negative chronotropic and dromotropic properties, the direct actions of bepridil on impulse formation and conduction in the sinus node become relevant. Disturbances in impulse formation or sino-atrial conduction as a result of drug administration might lead to sinus node dysfunction and related cardiac arrhythmias [15,76]. It was the purpose of the present study to investigate the direct effects of bepridil on impulse formation and conduction properties in the isolated rabbit sinus node, and to compare these effects with the influences on conduction and refractoriness in the atrial myocardium.

\section{Methods}

\section{The preparation}

Young New Zealand rabbits of either sex and weighing 1.5-2 kg were killed by cervical dislocation. After midsternal thoracotomy the heart was quickly excised and immersed in warmed oxygenated Tyrode solution for further dissection. A preparation was made including the sinus node region and right atrial free wall, and was transferred into a tissue-bath where it was superfused with warmed oxygenated Tyrode solution. For a detailed description of the isolation procedure and composition of the Tyrode solution see the 'Methods' section in chapter III. 


\section{Quantification of conduction properties and experimental protocol}

Impulse conduction through the border zone and center of the sinus node was studied by making an incision in the atrial part of the preparation ending in the sinus node. By means of extracellular surface electrodes the conduction time of stimulated atrial impulses through the sinus node could be measured. For a detailed description of this technique see 'Methods' section in chapter III.

In the present experiments the following parameters were measured: spontaneous sinus interval, transnodal conduction time during incremental atrial pacing with intervals ranging from $330 \mathrm{~ms}(3 \mathrm{~Hz})$ to the shortest cycle length at which 1:1 conduction still occurred (interval $F_{\max }$ ); conduction times of premature stimuli while shortening the coupling interval to the preceding basic stimulus; the earliest premature stimulus $\left(S_{1}-S_{2}\right)$ still conducted through the sinus node (effective refractory period). Premature atrial stimuli were delivered after every $15^{\text {th }}$ basic stimulus ( 330 ms intervall). The intensity of basic and premature stimuli was twice and four times diastolic threshold respectively. After completion of control measurements, increasing dosages of bepridil were added to the Tyrode solution with intervals of 45 minutes. The drug concentrations studied were $1 \times 10^{-6}, 2 \times 10^{-6}, 2.5 \times 10^{-6}$ and $3 \times 10^{-6} \mathrm{M}$. After each elevation of the drug concentration a stabilization period of 20 minutes was considered before measurements were repeated. In 11 experiments the effects of bepridil on the conduction properties of the sino-atrial border zone were tested. In 9 experiments the influences of bepridil on conduction through the center of the sinus node were investigated. In addition, in 8 of these experiments the effects of bepridil on atrial conduction properties were examined. Therefore an additional surface electrode was positioned on the stimulated atrial half, in between the stimulation electrode and the surface electrode positioned at the entrance of the sinus node bridge (right upper scheme in figure 4.1). Thus the conduction time between two atrial electrodes could be measured. The distance between these electrodes was determined microscopically with an accuracy of $0.1 \mathrm{~mm}$ and varied in the present experiments between 4 and $5 \mathrm{~mm}$.

Finally, in 4 experiments the effects of bepridil on the action potential configuration in sinus node fibers were studied. Under control conditions and after 60 minutes superfusion with $2 \times 10^{-6} \mathrm{M}$ bepridil a number of 10 intracellular recordings were obtained from fibers in the border zone followed by an equal number of registrations in the center of the sinus node. Standard microelectrode techniques were used (glass capillaries filled with $3 \mathrm{M} \mathrm{KCl}$; tip resistance 10-30 MOhm). The recorded signals were stored on magnetic tape (Ampex PR 2200) for off-line analysis. Action potential amplitude, action potential duration at the $50 \%$ level of the amplitude 
(APD50), maximal diastolic potential, $\mathrm{dV} / \mathrm{dt}$ max and the rate of spontaneous diastolic depolarization were measured in all recording sites.

From preliminary experiments it appeared that the actions of bepridil on atrial conduction properties were less potent than the influences on conduction properties of the sinus node. Therefore, after reaching the highest drug concentration tested in the sinus node $\left(3 \times 10^{-6} \mathrm{M}\right)$, the concentration was elevated to $4 \times 10^{-6} \mathrm{M}$ and to $6 \times 10^{-6} \mathrm{M}$ to study the effects on conduction and refractoriness in the atrium.

If conduction block occurred in the sinus node or atrial myocardium during basic pacing with an interval of $330 \mathrm{~ms}(3 \mathrm{~Hz})$, the conduction velocity was assumed to be zero. The normalized effects given in the diagrams are calculated from paired observations only; the absolute data given in the tables represent the average of all available observations. Statistical analysis was performed by means of the (paired, two-tailed) Student-t-test.

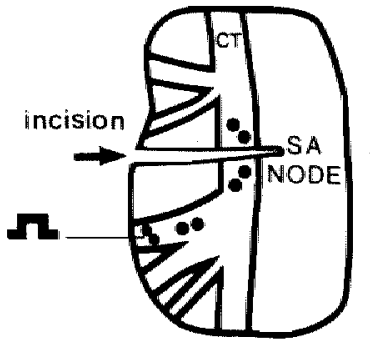

\section{Figure 4.1:}

Left upper panel: schematic representation of the preparation and principles of the method. The dual dots represent the position of blpolar surface electrodes. Right upper panel: typical effects of bepridll on conductlon through the border zone. Right lower panel: effects on conduction through the modal center. In both graphs the conduction time is given as function of the pacing interval, under control conditions (closed circles) and after administration of bepridill in concentratlons of $2 \times 10^{-6} \mathrm{M}$ (squares), $2.5 \times 10^{-6} \mathrm{M}$ (trlangles) and $3 \times 10^{-6} \mathrm{M}$ (asterlsks).
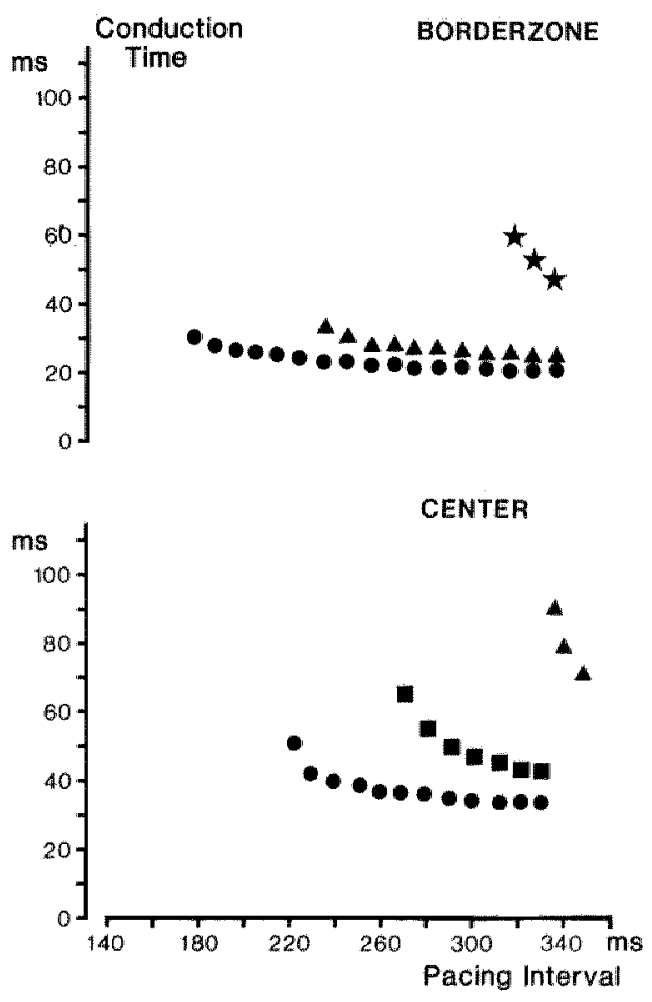


\section{Results}

\section{The effects of bepridil on impulse conduction through the sino- atrial border zone}

A typical example of the effects of bepridil on conduction through the sino-atrial border zone is shown in the upper right graph of figure 4.1. Under normal conditions slow atrial pacing with an interval of $330 \mathrm{~ms}$ resulted in a transnodal conduction time of $20 \mathrm{~ms}$ (circles). Decreasing the pacing interval below $210 \mathrm{~ms}$ revealed a rate-dependent lengthening of the conduction time until the shortest pacing interval of $170 \mathrm{~ms}$ was attained. At this point the conduction time had become $30 \mathrm{~ms}$. Bepridil in a concentration of $2.5 \times 10^{-6} \mathrm{M}$, caused an evident prolongation of the conduction time and the minimal pacing interval. Elevation of the drug concentration to $3 \times 10^{-6} \mathrm{M}$ caused a drastical suppression of conduction through the border zone. The conduction time during pacing with an interval of $330 \mathrm{~ms}$ was prolonged to $47 \mathrm{~ms}$, whereas the minimal pacing interval had become $310 \mathrm{~ms}$. Furthermore, the rate-dependent increase in conduction time was already present at low pacing rates and exhibited a much steeper slope than under control conditions.

\section{The effects of bepridil on conduction through the center of the sinus node}

The lower right diagram in figure 4.1 shows an example of the effects of bepridil on conduction through the center of the sinus node. Under control conditions the transnodal conduction time was $35 \mathrm{~ms}$ during pacing with a cycle length of $330 \mathrm{~ms}$. A rate-dependent increase in conduction time appeared with pacing intervals shorter than $280 \mathrm{~ms}$. The minimal pacing interval in this example was $220 \mathrm{~ms}$ with a conduction time of $55 \mathrm{~ms}$. Obviously, conduction of the impulse through the center of the sinus node was slower and characterized by a longer interval $F_{\max }$ than in the border zone. Bepridil in a concentration of $2 \times 10^{-6} \mathrm{M}$ caused a drastical increase in conduction time through the center of the sinus node. The minimal pacing interval was prolonged from 220 to $270 \mathrm{~ms}$ and showed an increase in conduction time of $15 \mathrm{~ms}$. This was in contrast to the sino-atrial border zone where this concentration hardly affected conduction of stimulated impulses. The effects of $2.5 \times 10^{-6} \mathrm{M}$ bepridil on conduction through the center of the sinus node were very marked. During pacing with an interval of $350 \mathrm{~ms}$ the conduction time was lengthened to $71 \mathrm{~ms}$ whereas the minimal pacing interval had become $330 \mathrm{~ms}$ with a conduction time of $90 \mathrm{~ms}$. Bepridil $3 \times 10^{-6} \mathrm{M}$ invariably caused conduction block in the nodal center. 
Apparently, bepridil depressed impulse conduction in the center of the sinus node to a greater extent than in the sino-atrial border zone.

\section{Comparison of the effects of bepridil on conduction properties in the sinus node and atrial myocardium}

In table 4.1 the average values of all conduction parameters are given as obtained under control conditions and after administration of bepridil in increasing concentrations. In figure 4.2 the normalized effects of bepridil on impulse conduction velocity during slow pacing ( $330 \mathrm{~ms}$ interval) are presented. Conduction velocity in the center of the sinus node (upper diagram) showed the highest sensitivity for the actions of bepridil: a minor but significant slowing in conduction was already observed with a drug concentration of $1 \times 10^{-6} \mathrm{M}$. This effect became more prominent at higher drug dosages. A marked reduction of the conduction velocity by $78 \%$ was found with a drug concentration of $2.5 \times 10^{-6} \mathrm{M}$. Bepridil $3 \times 10^{-6} \mathrm{M}$ invariably caused conduction block in the center of the sinus node. In the border zone of the

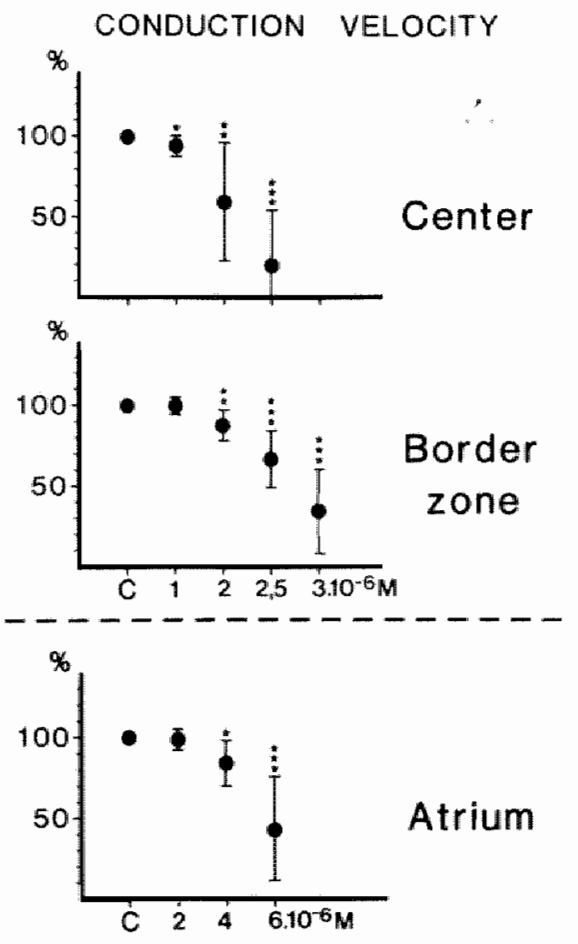

Figure 4.2:

The normalized effects (mean \pm SD) of beprildil on conductlon vilocity during slow pacing $(390 \mathrm{~ms}$ intervall) in the nodal center (upper dlagram), the slinoatrial border zone (middle diagram) and the atrial myocardlium (lower diag. ram). Notice the different concentrations tested in the sinus node and atrium. $\mathrm{C}=$ Control. Significance (palred) to control: * $\mathrm{p}<0.05 ; * * \mathrm{p}<0.01$; $* \star * * 0<0.001$ 
sinus node (middle diagram) the average effects were less dramatic: bepridil in a concentration of $3 \times 10^{-6} \mathrm{M}$ reduced conduction velocity by $65 \%$, whereas a drug concentration of $1 \times 10^{-6} \mathrm{M}$ did not affect conduction velocity at all. In the atrium (lower diagram) bepridil exhibited a significant negative dromotropic action as well, although at drug concentrations which caused complete conduction block in the sinus node.

In figure 4.3 the average effects of bepridil on tissue refractoriness - represented by the effective refractory period (left) and interval $F_{\max }$ (right) - are given. In the center of the sinus node (upper diagrams) bepridil in a concentration of $1 \times 10^{-6} \mathrm{M}$ prolonged both the effective refractory period and interval $F_{\max }$ significantly. A drug concentration of $2.5 \times 10^{-6} \mathrm{M}$ prolonged both parameters dramatically, by $81 \%$ and $67 \%$ respectively. In the border zone the effects of bepridil on refractoriness were only slightly less than in the center of the sinus node (middle diagrams). Bepridil caused also a significant prolongation of the effective refractory period and interval Fmax in the atrium, although with concentrations twice as high as in the sinus node.

ERP
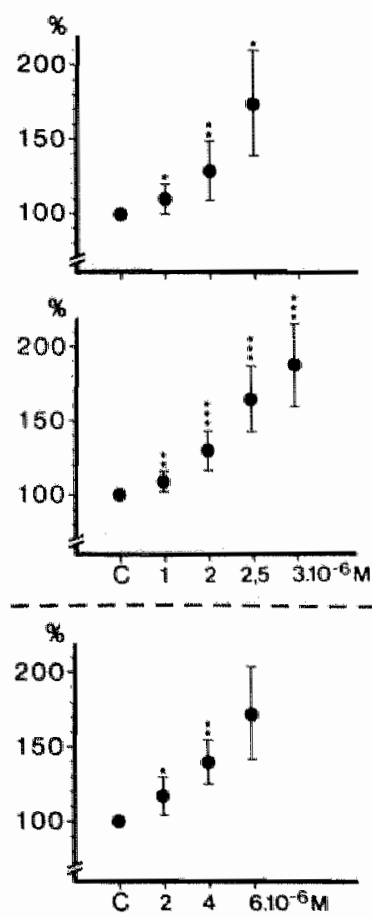

INTERVAL Fmax
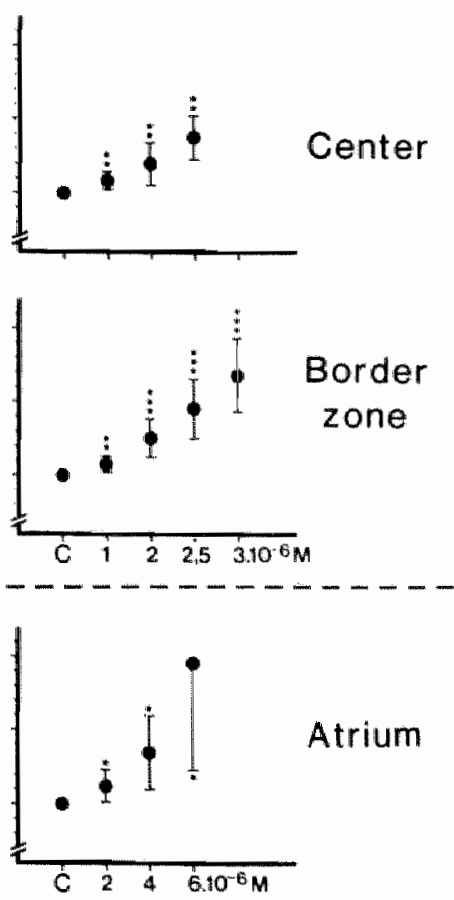

Figure 4.3:

The normalized effects (mean $\pm \mathrm{SD}$ ) of bepridil on the effective refractory perlod (left diagrams) and the Interval $F_{\max }$ (right dlagrams) in the nodal center (upper diagrams), the sino-atrial border zone (middle dlagrams), and atrial myocardium (lower dlagrams), $\mathrm{C}=$ Control. Significance (paired) to control: * $p<0.05$, ** $\mathrm{p}<0.01$, $* * * p<0.001$ 
$\varphi$
0
0
0

옿 $\quad$ 인 $\bar{\alpha}$

0

N

$\stackrel{\infty}{\sim}$

$\stackrel{+1}{+1}$
ถ⿻ำ

H

$\stackrel{N}{=}$

$\stackrel{\infty}{\stackrel{2}{+}}$

$+1$

$\overline{\underline{I}}$

ल) $\frac{\infty}{+1}$

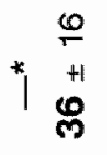

$g$
*
+1
$\frac{0}{m}$

$\mathscr{N} \div$

(1)

品

$\frac{1}{8}$

क

$\stackrel{g}{5}$

용 여

9

$+1+1$

昌 㟧

능

$+11+1$

心ํㅜㅇ

8

요

$\wedge$

H H

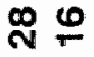

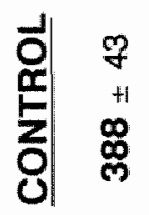

$\omega+\varphi$

茫市

满

H H H

ํํํ

$\stackrel{8}{\circ}$

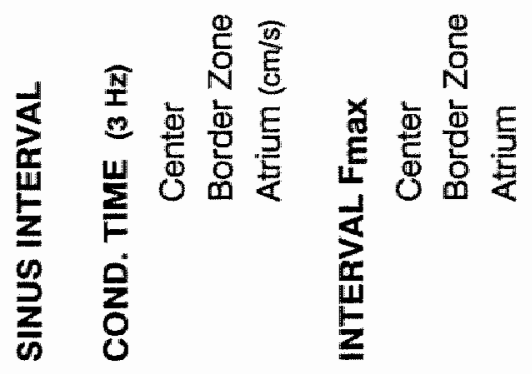

党

$\stackrel{+}{\stackrel{0}{*}}$

$\pm$

it

tᄒ

लै

* $\frac{+1}{N}$

苗 ले

$+1+1$

巡 으

以

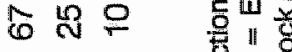

H H H

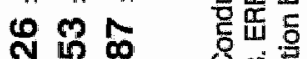

N $-\infty \infty$

क을 흘

이망

$+1+5$

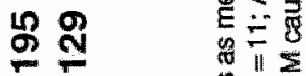

E $=0$

둗ㅇ

ㅊำ

+1 +1 के क्ष

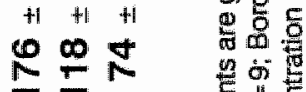
垔 II

동

ख 而 들 语要 क्ष 농 $\frac{\pi}{0}$ 总要 高毫 
In contrast to the marked influences on conduction through the sinus node, bepridil influenced sinus automaticity only to a limited extent. This is illustrated by the graph in figure 4.4 (see also table 4.1). On the average, bepridil in a concentration of $3 \times 10^{-6} \mathrm{M}$ caused a $7 \%$ reduction in sinus rate. However, with this drug concentration the morphology and time relation between both atrial electrograms often changed, suggesting the occurrence of a pacemaker shift within the sinus node. When the drug concentration was raised above $3 \times 10^{-6} \mathrm{M}$, sinus rate suddenly decreased, followed by an abrupt cessation of spontaneous activity, mostly before a drug concentration of $6 \times 10^{-6} \mathrm{M}$ was reached. In this stage intracellular recordings from fibers in the center of the sinus node showed that spontaneous action potential gieneration was degenerated to subthreshold oscillations. A typical example of this phenomenon is shown in the lower part of figure 4.4. No evidence was obtained for the occurrence of ectopic impulse formation in the atrial myocardium.

\section{The effects of bepridil on action potential configuration}

In 4 experiments we studied the influences of $2 \times 10^{-6} \mathrm{M}$ bepridil on the configuration of action potentials from fibers in the sinus node. A number of 10 intracellular recordings were made both in the sino-atrial border zone and nodal center. In table 4.2 the normalized changes in action potential parameters after $60 \mathrm{~min}$ action of bepridil are summarized. In figure 4.5 typical examples of the changes in action poten-

\section{SINUS RATE}

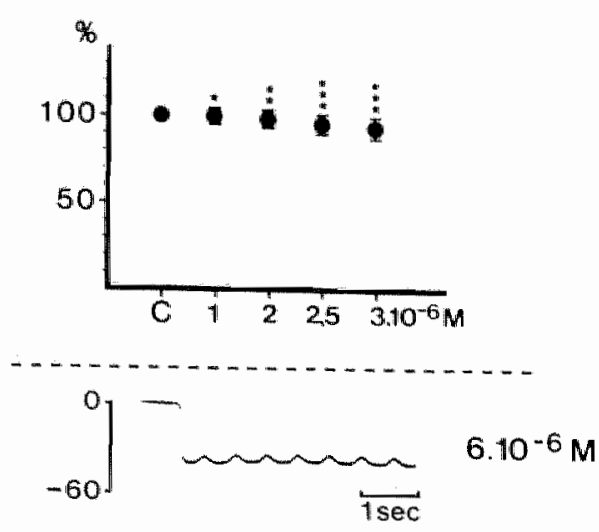

\section{Figure 4.4:}

The normalized effects (mean \pm SD) of bepridll on sinus rate. The lower part of the figure shows an intracellular registration from a fiber in the center of the sinus node after sudden cessation of sinus automaticity with bepridil $6 x$ $10^{-6} \mathrm{M}$. Only subthreshold oscillations around a membrane potential of about $-40 \mathrm{mV}$ were observed. $\mathrm{C}=$ Control. Significance (palred) to control: * $p<0.05$, ${ }^{* *} p<0.01,{ }^{* * *} p<0.001$ 
Table 4.2

Effects Of $60 \mathrm{Min}$ Bepridil $2 \times 10^{-6} \mathrm{M}$ On Action Potential Configuration In The Sinus Node

$n=4$ BORDER ZONE (\%) CENTER $(\%)$

Action Potential Amplitude $-21 \pm 18$ $-20 \pm 3 * *$ Maximal Diastolic Potential $-7 \pm 7$ $-14 \pm 5^{* *}$ Action Potential Duration (APD50) $+17 \pm 3^{* *}$ $+21 \pm 5^{* *}$ $\mathrm{dV} / \mathrm{d} \mathrm{t}_{\max }$

Spontaneous Diastolic $-44 \pm 19$ * $-53 \pm 21 *$

Depolarization

In each $(n=4)$ experlment 2 serles of 10 Intracellular recordings were made in the border zone and nodal center, before and after administration of beprldil. Significance (paired) compared to control: ${ }^{\star} \mathrm{p}<0.05 ;{ }^{* *} \mathrm{p}<0.01$.

tial configuration are shown. In the border zone of the sinus node the maximal rate of phase-0 depolarization ( $\mathrm{dV} / \mathrm{dlt}$ max) was reduced markedly whereas the action potential duration (APD50) showed a significant increase. In the center of the sinus node equivalent effects were found. Only the spontaneous diastolic depolarization rate remained unaffected in both regions. Notice that the action potentials from the center of the sinus node showed a more abrupt transition to the upstroke after addition of bepridil. This suggests a shift of the pacemaker to another position in the sinus node.

\section{Discussion}

The results of the present experiments demonstrate that the direct negative chronotropic action of bepridil is preceded by a marked depression of the conduction properties in the isolated sinus node. Bepridil decreased the conduction velocity of impulses in the border zone or center of the sinus node in a dose-dependent manner. This negative dromotropic effect was progressive during incremental pacing (figure 4.2). As a result, the physiological range of heart rates showing a con- 
BORDER
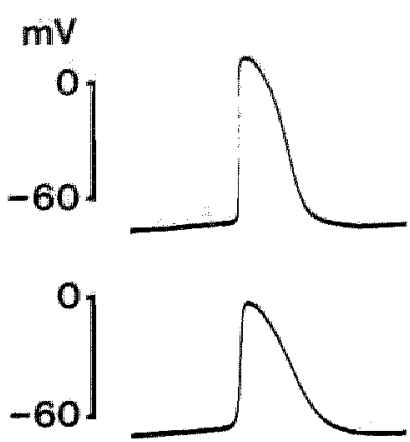

\section{CENTER}

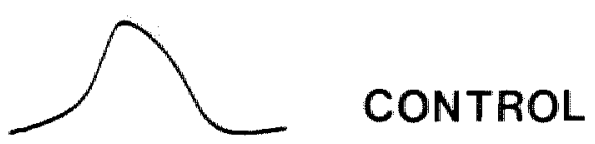

BEPRIDIL

$2.10^{-6} \mathrm{M}$

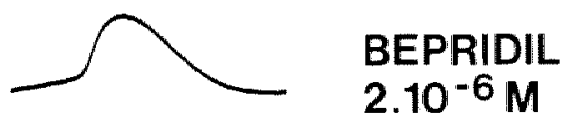

$\overline{100 \mathrm{~ms}}$

\section{Figure 4.5:}

Typical examples of action potentials recorded from fibers in the border zone and center of the sinus node, before and after $60 \mathrm{~min}$ activity of bepridil $2 \times 10^{-6} \mathrm{M}$. Notice the changes in action potentlal amplitude, duratlon and upstroke velocity. See also table 4.1.

sistent transnodal conduction velocity was narrowed. In the center of the sinus node conduction was depressed with lower drug concentrations than in the sino-atrial border zone. The findings of the present experiments are in agreement with the results of other investigators who studied the effects of bepridil on AV nodal conduction $[47,79,131,229]$. On the other hand, Kobayashi and coworkers studied the effects of bepridil on sino-atrial conduction in denervated dogs but could not find any significant effect [139]. This might be explained by the indirect method used to measure the sino-atrial conduction time (chapter I), or by alterations in autonomic tone due to changes in hemodynamics after administration of bepridil.

In several studies it was found that bepridil decreases the slow calcium inward current in nodal cells $[17,126,277]$. This activity explains the depression of conduction velocity in the center of the sinus node as found in the present experiments. It also explains the decrease in conduction velocity in the sino-atrial border zone. In this region of the sinus node the phase- 0 depolarization is based on a combination of the slow calcium and fast sodium inward current $[23,139,145,161,253]$. In the present experiments bepridil exhibited a negative dromotropic effect on atrial fibers as well. This strongly suggests an additional depressive action of bepridil on the fast sodium inward current. Therefore, depression of conduction through the sino-atrial border zone is probably based on a reduction of both the fast and slow inward currents in these fibers. 
In addition to the influences on impulse conduction velocity, bepridil also prolonged the refractory period in the sinus node. Both the effective refractory period and the interval $F_{\text {max }}$ were increased markedly. In contrast to the effects on conduction velocity, bepridil caused a similar prolongation of tissue refractoriness in the border zone and center of the sinus node. In the atrium the effective refractory period and the interval $F_{\max }$ were lengthened as well, although with drug concentrations twice as high as in the sinus node. A prolonging influence of bepridil on the refractory period of atrial, ventricular and AV nodal fibers was also observed in other studies $[11,47,79,129,131,229]$. However, the lengthening action on refractoriness in the isolated rabbit sinus node has, to our knowledge, not been reported yet. In the present experiments lengthening of the refractory period in the sinus node was accompanied by an increase of the action potential duration in nodal fibers. This was also described by other investigators $[17,126,129]$. However, the mechanism underlying this increase in action potential duration remains unclear. The slow-channel blocking features of bepridil will shorten the plateau-phase of the action potential [273]. On the other hand, it was demonstrated that a decrease in cellular $\mathrm{Ca}^{2+}$-influx causes a prolongation of the action potential duration [195,241]. A decrease of the potassium outward current, due to a diminished intracellular $\mathrm{Ca}^{2+}$-concentration, might be responsible for this prolongation [83]. Finally , a reduction of the action potential upstroke velocity leads to a prolonged phase-0 depolarization. This results in an increased action potential duration. Bepridil is known to cause a decrease of the membrane responsiveness in Purkinje fibers. In other fiber types this effect might be present as well and cause an additional reduction of the phase- 0 depolarization rate in case of early premature beats, delivered to cletermine the effective refractory period [129].

In the present experiments tissue refractoriness was represented by the effective refractory period and minimal pacing interval or interval Fmax. It should be stressed that these parameters represent different electrophysiological conditions. This is illustrated by the fact that a single premature impulse can be conducted through the sinus node with a higher degree of prematurity and a lower conduction velocity than a train of rapidly succeeding impulses (chapter III). The maximal pacing rate $\left(F_{\max }\right)$ determines the cycle length of sustained sinus node reentrant tachycardias.

The marked effects of bepridil on intranodal conduction properties contrasted with the limited effects on sinus rate. At drug concentrations which did cause a decrease ( 4 to $6 \times 10^{-6} \mathrm{M}$ ) in sinus rate, impulse conduction through the sinus node was completely blocked. Finally, impulse formation suddenly ceased and degenerated into subthreshold oscillations. This phenomenon was also observed by other authors, using similar drug concentrations as in the present experiments $[17,90,126]$. 
The fact that bepridil depresses intranodal conduction properties prior to sinus rate, and within a relatively narrow concentration range, is an important finding with clinical implications. The therapeutic plasma concentrations in man resemble those used in the present experiments $[9,68]$. Underestimation of the drug effects might occur if only the heart rate is used as a control index. Intoxication might lead to unexpected sinus exit block. On the other hand, extrapolation of the present findings to the in vivo situation should be done with reservation because of the additional hypotensive and negative inotropic effects of bepridil, caused by its calcium antagonistic activity. These actions will modulate sinus function indirectly by changes in autonamic tone.

From the present study it was concluded that: (1) bepridil in concentrations between $1 \times 10^{-6} \mathrm{M}$ and $3 \times 10^{-6} \mathrm{M}$ decelerates impulse conduction and prolongs refractoriness in the isolated rabbit sinus node prior to any effect on sinus automaticity: (2) this effect is more marked in the center of the sinus node than in the sinoatrial border zone; (3) bepridil in concentrations between $4 \times 10^{-6} \mathrm{M}$ and $6 \times 10^{-6} \mathrm{M}$ depresses impulse conduction and prolongs the refractory period in the atrium, whereas it abolishes sinus automaticity; (4) bepridil probably exerts a direct inhibitory effect on both the fast sodium inward current and the slow calcium inward current, of which the latter is most sensitive. 


\section{Chapter V}

EFFECTS OF VERAPAMIL, DILTIAZEM AND

DISOPYRAMIDE ON SINUS

FUNCTION: A COMPARISON WITH BEPRIDIL

Eur J Pharmacol 160,369-376, 1989 



\section{Introduction}

Cardiac drugs demonstrating negative chronotropic actions may affect conduction of the impulse in the sinus node as well. Therefore, to determine the actions of a drug on sinus node function, the effects on sino-atrial conduction properties should be studied in addition to the influence on sinus automaticity. In previous experiments the influences of bepridil on sinus function were investigated (chapter IV). This drug appeared to affect sino-atrial conduction and sinus automaticity sequentially. In the border zone of the sinus node impulse conduction velocity was markedly depressed and refractoriness drastically prolonged with bepridil in concentrations which did not affect sinus rate and atrial conduction properties. These results demonstrated the possibility of a sequential mode of onset of different drug effects. To compare the profile of actions of bepridil with those of other drugs, we studied the effects of verapamil, dilitiazem and disopyramide on conduction properties in the isolated rabbit sinus node and atrial myocardium. These drugs all exhibit negative chronotropic effects $[41,127,130,139,257]$. Verapamil and diltiazem are two typical class IV antiarrhythmic drugs, whereas disopyramide is classified as a class I drug. From the present experiments it appeared that each drug possesses a typical profile of action on sinus function and atrial conduction properties. The results of these experiments are described in the present chapter.

\section{Methods}

Young New Zealand rabbits of either sex and weighing $1.5-2 \mathrm{~kg}$ were killed by cervical dislocation. Via midsternal thoracotomy the heart was quickly excised and immersed in oxygenated Tyrode solution for further dissection. A preparation including the sinus node region and attached atrial myocardium was made, transferred into a tissue-bath, and superfused with Tyrode solution at a temperature of $38^{\circ}$ Celsius. For a detailed description of the isolation procedures and composition of the Tyrode solution see the 'Methods' section in chapter III. We studied the effects of verapamil, diltiazem and disopyramide on conduction properties in the border zone of the sinus node and in the right atrial myocardium. The model described in chapters III and IV was used. Atrial conduction and refractoriness in the atrium was measured between an additional atrial surface electrode, positioned at the stimulated atrial half, and the proximal electrode on top of the crista terminalis.

In preliminary experiments a specific concentration range (four dosages) was determined for each drug whereby the highest concentration should demonstrate an apparent influence on impulse conduction through the border zone of the sinus 
node. Thus we decided to study verapamil in concentrations of $2.2,4.4,6.6$ and 8.8 $\times 10^{-8} \mathrm{M}$. Since this was a relatively narrow concentration range, we also examined the effects of 13.2 and $17.6 \times 10^{-8} \mathrm{M}$ on atrial conduction properties. Diltiazem was studied in concentrations of $5 \times 10^{-7}, 1 \times 10^{-6}, 3 \times 10^{-6}$ and $5 \times 10^{-6} \mathrm{M}$, both in the atrium and in the border zone of the sinus node. Disopyramide was examined after addition of $1 \times 10^{-6} \mathrm{M}$ atropine to the superfusate to avoid interference between the direct effects on sinus function and the known anticholinergic actions of the drug $[130,181]$. The concentrations studied were $1 \times 10^{-6}, 5 \times 10^{-6}, 1 \times 10^{-5}$ and $5 \times 10^{-5}$ $M$, both in the atrium and border zone of the sinus node.

The electrophysiological parameters measured were: spontaneous sinus interval; conduction time during regular pacing with intervals ranging from $330 \mathrm{~ms}(3 \mathrm{~Hz})$ to the shortest interval which was still conducted in a 1:1 manner through the sinus node or atrium (interval $F_{\max }$ ); conduction times of premature stimuli, while decreasing the coupling interval to the preceding basic stimulus in steps of $5 \mathrm{~ms}$ to determine the effective refractory period. Premature stimuli were delivered after every $15^{\text {th }}$ basic beat $(3 \mathrm{~Hz})$. The stimulus strength was adjusted to twice diastolic threshold during regular pacing and four times threshold in case of premature stimulation. The entire pacing protocol was executed once to obtain control values. Subsequently, the drug to be studied was added to the Tyrode solution. Diltiazem and disopyramide requested a stabilization period of less than $10 \mathrm{~min}$. Therefore, measurements during superfusion with one of these drugs were started 20 min after drug administration. The drug concentration was raised with intervals of $45 \mathrm{~min}$. In case of verapamil a stabilization period of about 45 min was necessary. Therefore, in these experiments measurements were started 60 min after drug administration. In this case the drug concentration was elevated with intervals of $75 \mathrm{~min}$.

If conduction block occurred in the border zone of the sinus node or in the atrium during pacing with an interval of $330 \mathrm{~ms}(3 \mathrm{~Hz})$, conduction velocity was assumed to be zero. In this case the effective refractory period could not be determined. The percentual effects of each drug given in the diagrams are calculated from paired observations only. The Student-t-test was used for statistical analysis of the data. 


\section{Results}

\section{Verapamil}

We studied the effects of verapamil on sinus rate and sino-atrial conduction properties in 10 experiments of which the results are depicted in figure 5.1. Verapamil in concentrations up to $4.4 \times 10^{-8} \mathrm{M}$ demonstrated no significant effect on sino-atrial conduction velocity during atrial pacing with a frequency of $3 \mathrm{~Hz}$. However, elevation of the drug concentration to $6.6 \times 10^{-8} \mathrm{M}$ or higher caused a significant reduction of the impulse conduction velocity in the border zone. The transnodal conduction time increased from $24 \pm 5 \mathrm{~ms}$ (mean $\pm \mathrm{SD}$ ) under control conditions to $28 \pm 5 \mathrm{~ms}$ with verapamil in a concentration of $6.6 \times 10^{-8} \mathrm{M}$ and to $32 \pm 9$ $\mathrm{ms}$ with a drug concentration of $8.8 \times 10^{-8} \mathrm{M}$. In one experiment second degree conduction block (2:1) developed with verapamil in the highest concentration.

Verapamil also caused a dose-dependent prolongation of refractoriness in the border zone of the sinus node. A drug concentration of $2.2 \times 10^{-8} \mathrm{M}$ which did not affect the sino-atrial conduction velocity, caused a significant increase of the minimal pacing interval (interval $F_{\max }$ ) and the effective refractory period by $15 \%$ and $12 \%$ respectively (figure 5.1 ). Verapamil in a concentration of $8.8 \times 10^{-8} \mathrm{M}$ prolonged

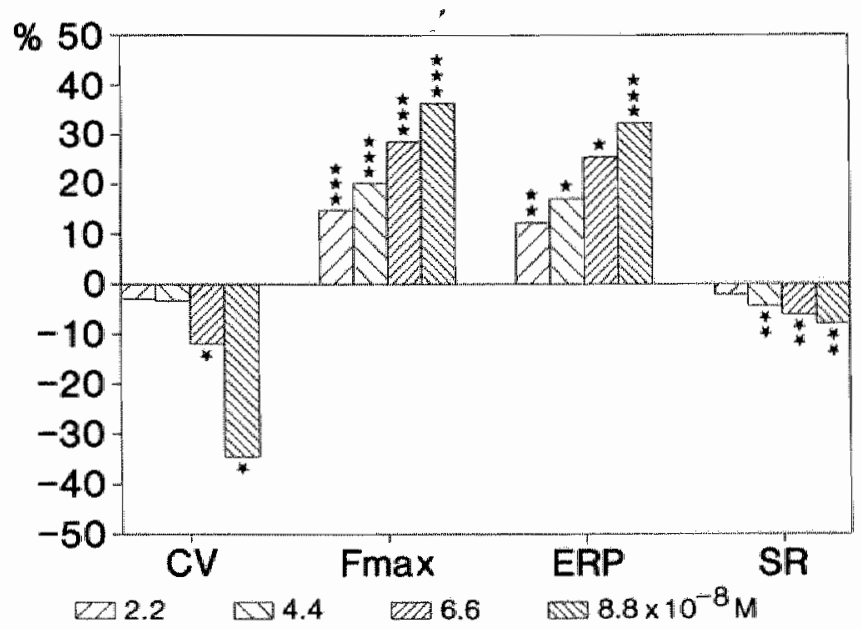

Figure 5.1:

The effects of verapamil on sinus function. $\mathrm{CV}=$ conduction velocity in the border zone during 3 $\mathrm{Hz}$ pacing; $F \max =$ Interval $F_{\text {max }}$; ERP = effectlve refractory period; $S A=$ sinus rate. See text for control values and standard deviations. Significance (paired) to control: ${ }^{*} p<0.05 ;{ }^{*} p<0.01$; $* * * \mathrm{p}<0.001$. 
the refractory period in the border zone of the sinus node markedly. The interval $F_{\max }$ was lengthened from $176 \pm 23 \mathrm{~ms}$ (control) to $239 \pm 53 \mathrm{~ms}(+36 \%)$ and the effective refractory period trom $119 \pm 15 \mathrm{~ms}$ to $156 \pm 39 \mathrm{~ms}(+32 \%)$.

The apparent effects of verapamil on conduction properties in the border zone of the sinus node were not accompanied by an equivalent depression of sinus automaticity. Verapamil in the present concentrations reduced sinus rate by less than $8 \%$, from an average interval of $360 \pm 25$ ms under control conditions to $393 \pm 46$ $m s$ with a drug concentration of $8.8 \times 10^{-8} \mathrm{M}$. In 6 experiments we studied the influences of verapamil on conduction properties in the atrium. It appeared that neither conduction velocity nor tissue refractoriness was affected significantly by verapamil in concentrations up to $17.2 \times 10^{-8} \mathrm{M}$.

\section{Diltiazem}

We studied the effects of diltiazem on sinus function in 10 experiments of which the average results are depicted in figure 5.2. Impulse conduction through the border zone of the sinus node during pacing with a frequency of $3 \mathrm{~Hz}$ was not significantly influenced by diltiazem in concentrations up to $3 \times 10^{-6} \mathrm{M}$. However, a drug concentration of $5 \times 10^{-6} \mathrm{M}$ caused a significant depression of the conduction vel-

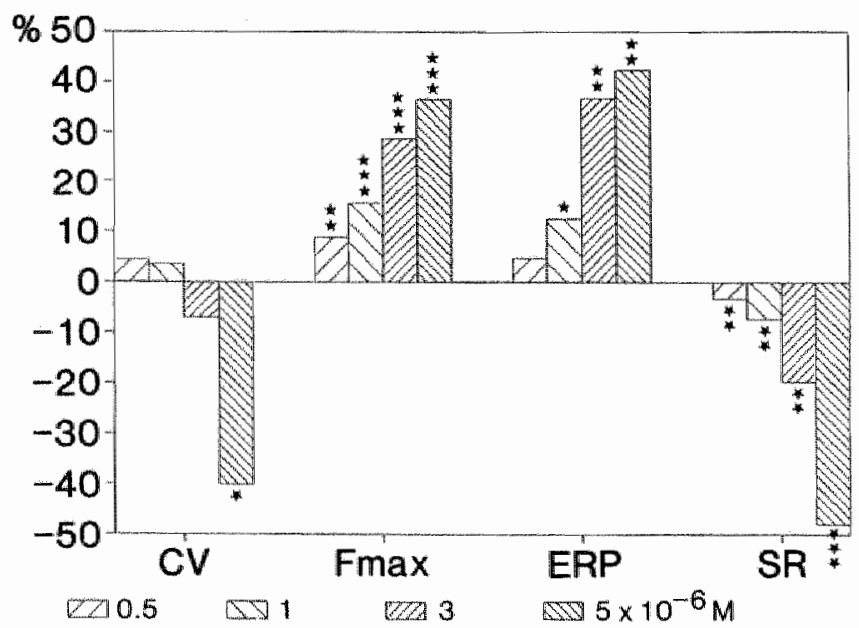

Figure 5.2:

The effects of dittlazem on sinus functlon. $\mathrm{CV}=$ conduction velocity in the border zone during 3 $\mathrm{Hz}$ pacing; $F \max =$ interval $F_{\text {max }} ; E R P=$ effective refractory period; $S R=$ sinus rate. See text for control values and standard deviatlons. Significance (paired) to control: ${ }^{*} p<0.05$; ${ }^{\star *} p<0.01$; $* * * p<0.001$. 
ocity by $-40 \%$ whereas in $30 \%$ of the experiments $1: 1$ conduction through the border zone failed. Sino-atrial refractoriness was influenced by ditiazem as well. A drug concentration of $1 \times 10^{-6} \mathrm{M}$ which did not affect sino-atrial conduction velocity, caused a significant prolongation of the interval $F_{\max }$ and of the effective refractory period in the border zone of the sinus node (figure 5.2). Higher drug concentrations caused a dose-dependent lengthening of both parameters. Diltiazem $5 \times 10^{-6} \mathrm{M}$ increased the interval $F_{\max }$ from $143 \pm 17 \mathrm{~ms}$ (control) to $189 \pm 37 \mathrm{~ms}$ and prolonged the effective refractory period from $95 \pm 10 \mathrm{~ms}$ (control) to $131 \pm 32 \mathrm{~ms}(\mathrm{n}=7)$. In contrast to verapamil, diltiazem in the present concentrations caused an equivalent depression of sino-atrial conduction properties and sinus rate. The sinus interval increased in a dose-dependent manner from $406 \pm 43$ ms under control conditions to $642 \pm 180 \mathrm{~ms}$ during superfusion with dittiazem in a concentration of $5 \times 10^{-6} \mathrm{M}$. Similar to verapamil, diltiazem did not exhibit significant effects on atrial conduction properties $(n=8)$.

\section{Disopyramide}

In 10 experiments we studied the effects of disopyramide on sinus function. Atropine in a concentration of $1 \times 10^{-6} \mathrm{M}$ was added to the Tyrode solution to avoid interference of the described anticholinergic actions of disopyramide $[130,181]$. Atropine exhibited no effects on sinus rate or sino-atrial conduction in the isolated sinus node. The results of the present experiments are depicted in the upper panel of figure 5.3. Disopyramide in a concentration of $1 \times 10^{-5}$ and $5 \times 10^{-5} \mathrm{M}$ caused a significant decrease in conduction velocity in the border zone of the sinus node. Conduction time during pacing with an interval of $330 \mathrm{~ms}(3 \mathrm{~Hz})$ increased from an average control value of $16 \pm 6 \mathrm{~ms}$ to $33 \pm 15 \mathrm{~ms}$ with disopyramide in a concentration of $5 \times 10^{-5} \mathrm{M}$. This apparent negative dromotropic action of more than $50 \%$ was accompanied by an even stronger effect on sino-atrial refractoriness. Disopyramide in a concentration of $5 \times 10^{-6} \mathrm{M}$ prolonged the interval $F_{\max }$ significantly from $156 \pm 20 \mathrm{~ms}$ (control) to $166 \pm 27 \mathrm{~ms}$ and the effective refractory period from $107 \pm 13 \mathrm{~ms}$ (control) to $121 \pm 16 \mathrm{~ms}$. These effects were dose-dependent and became very marked with the highest drug dosage of $5 \times 10^{-5} \mathrm{M}$ : the interval $F_{\max }$ increased by $66 \%$ from $156 \pm 20 \mathrm{~ms}$ (control) to $258 \pm 47 \mathrm{~ms}$ and the effective refractory period by $112 \%$ from $107 \pm 13 \mathrm{~ms}$ (control) to $223 \pm 53 \mathrm{~ms}$. Disopyramide caused a significant decrease in sinus rate. However, this effect was less prominent than the depressive actions on sino-atrial conduction properties. Disopyramide in a concentration of $5 \times 10^{-5} \mathrm{M}$ prolonged the spontaneous interval from $387 \pm 57$ $\mathrm{ms}$ (control) to $479 \pm 43 \mathrm{~ms}$ being a significant negative chronotropic effect of $20 \%$. 

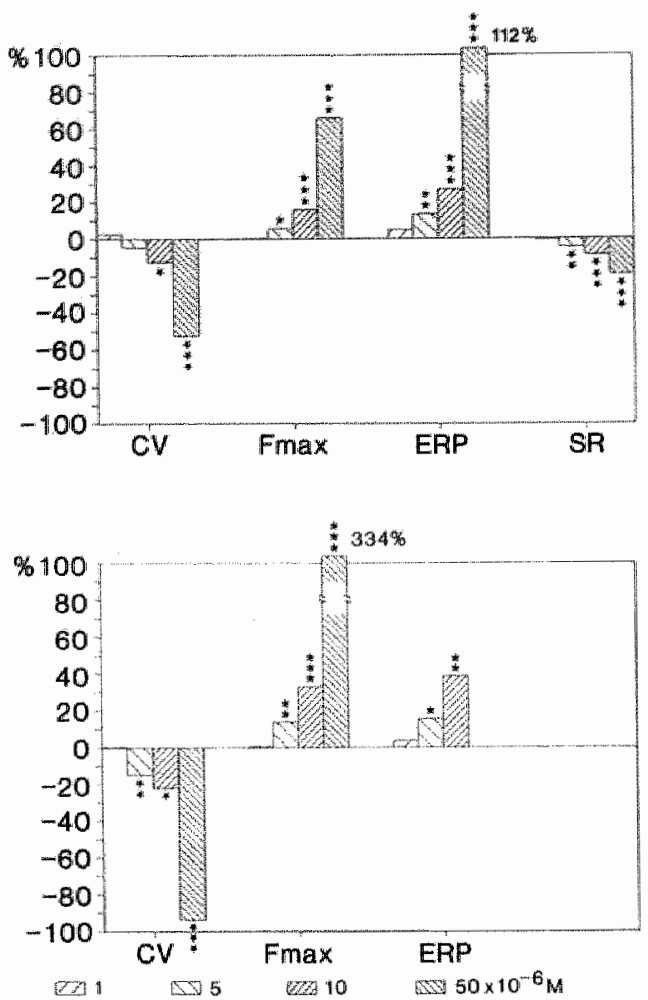

\section{Figure 5.3:}

Upper panel: The effects of verapamil on sinus function. $\mathrm{CV}=$ conduction velocity in the border zone during $3 \mathrm{~Hz}$ pacing; Fmax $=$ interval $F_{\text {max }} ;$ ERP $=$ effective refractory period: $\mathrm{SR}=$ sinus rate. See text for control values and standard deviations.

Lower panel: The actions of disopyramide on conduction properties in the right atrial myocardium. Notice: different ordinates in comparison to figures 5.1 and 5.2. Significance (paired) to control: * $p<0.05^{* *} p<0.01$; $* * * p<0.001$

In 5 experiments we studied the influence of disopyramide on atrial conduction. The results of these experiments are summarized in the lower panel of figure 5.3. Disopyramide in a concentration of $5 \times 10^{-6} \mathrm{M}$ and $1 \times 10^{-5} \mathrm{M}$ reduced the average atrial conduction velocity during slow pacing $(3 \mathrm{~Hz})$ from $52 \pm 17 \mathrm{~cm} / \mathrm{s}$ (control) to $48 \pm 18$ and to $41 \pm 15 \mathrm{~cm} / \mathrm{s}$ respectively. Elevating the concentration to $5 \times 10^{-5} \mathrm{M}$ resulted in $2: 1$ conduction block during slow pacing $(3 \mathrm{~Hz})$ in $80 \%$ of the cases. The refractory period in the atrial myocardium was drastically prolonged by disopyramide. A drug concentration of $1 \times 10^{-5} \mathrm{M}$ prolonged the interval $F_{\text {max }}$ from $89 \pm$ $8 \mathrm{~ms}$ (control) to $118 \pm 14 \mathrm{~ms}$ and the effective refractory period from $61 \pm 13 \mathrm{~ms}$ to $85 \pm 19 \mathrm{~ms}$. Disopyramide $5 \times 10^{-5} \mathrm{M}$ caused conduction block in the atrium in 2 out of 5 experiments. As a consequence, the interval $F_{\max }$ could be determined in only 3 out of 5 preparations and amounted to $381 \pm 40 \mathrm{~ms}$. The effective refractory period during $3 \mathrm{~Hz}$ basic pacing could not be measured anymore because of $2: 1$ conduction block in 4 out of 5 experiments. 


\section{Discussion}

In the present study we investigated the direct effects of verapamil, diltiazem and disopyramide on conduction and refractoriness in the isolated sinus node and right atrial myocardium in order to compare these actions with the effects of bepridil as found in previous experiments (chapter IV). Bepridil was introduced as a calciumantagonistic compound, but our experiments as well as other studies brought evidence for additional fast-channel blocking activity $[79,129]$. Verapamil and diltiazem are type I calcium antagonists exhibiting direct effects on sinus and AV nodal fibers [238,244]. Disopyramide belongs to the group of quinidine-like drugs and influences predominantly atrial and ventricular fibers. However, disopyramide also reduces heart rate and is suggested to affect sinus function both in a direct and indirect manner $[21,257,130,181]$.

To compare the profiles of actions of drugs in vitro, the compounds can be tested in equal concentrations. However, this may lead to a situation in which one drug is studied at its minimal effective concentration while the other drug is tested at toxic dosages. In the first case this might result in minor or even indetectable effects, disregarded because of statistical insignificance and leading to underestimation of the potency of the drug. In the second case sequential onsets of different drug effects as found with bepridil might be missed. Therefore, in the present study we chose a concentration range for each drug of which the lower limit exerted almost no effect on sino-atrial conduction velocity and refractoriness, whereas the highest dosage caused a marked - about $50 \%$ - but not toxic effect on these parameters.

The conventional techniques to study sino-atrial conduction properties are based on the atrial return cycle after programmed atrial stimulation [191,254]. However, the post-extrasystolic or post-pacing sinus impulse might arise from other locations in the sinus node than during stable sinus rhythm and might be influenced electrotonically by the premature atrial activation. These phenomena influence sinoatrial conduction and make the estimated SACT less accurate than expected theoretically $[30,249]$. Furthermore, drugs which exhibit chronotropic actions may cause intranodal pacemaker shifts too and affect the estimated SACT, not because of direct dromotropic activity but simply because of a changed sino-atrial conduction route. The method used in the present experiments is independent of sinus automaticity (see also chapters I and III).

In fibers of the sinus node and AV node the slow calcium inward current plays an important role in the generation of action potentials. Blockade of the calcium inward current by verapamil or other calcium antagonists will influence automaticity and conduction in these structures $[80,103,288,296]$. In a clinical study Breithardt and 
coworkers [41] found a significant prolongation of the estimated sino-atrial conduction time after intravenous administration of verapamil. Other investigators found a negative dromotropic action on AV nodal conduction with this drug $[146,259,288]$. EXperimental studies established that verapamil depresses sinus automaticity direct$\mathrm{ly}$; it reduces action potential amplitude, maximal diastolic potential ${ }_{\text {, }} \mathrm{dV} / \mathrm{dt}$ max and spontaneous diastolic depolarization in nodal fibers $[132,142,288]$. This is in agreement to the results of the present experiments which demonstrate that verapamil reduces impulse conduction velocity in the border zone of the isolated sinus node. An important finding is the relative dominance of this effect: similar to bepridil, verapamil caused a marked depression in sino-atrial conduction without a simultaneous and equivalent reduction in sinus rate. However, verapamil exhibited these negative dromotropic effects at considerable lower drug concentrations than bepridil. Direct depression of sino-atrial conduction by verapamil $\left(1 \times 10^{-5} \mathrm{M}\right)$ was also described by Jordan and coworkers [121] who found an increase in antegrade sino-atrial conduction time in the isolated rabbit sinus node. Kawai and colleagues [132] described the occurrence of intranodal conduction block and an average sinus slowing of $57 \%$ with verapamil $4.4 \times 10^{-6} \mathrm{M}$. In the present experiments verapamil required a relatively long period of exposure until a steady state was attained. This was also described by other investigators $[69,128]$. In contrast to bepridil, verapamil did not affect conduction velocity and refractoriness in the atrium. This indicates that verapamil has no fast-channel inhibitory features in the present concentration range.

Diltiazem, the other typical calcium antagonist, demonstrated similar depressive actions on sino-atrial conduction properties as verapamil and bepridil. The sino-atrial conduction velocity was reduced and the refractory period prolonged in a doserelated manner. However, unlike verapamil and bepridil, diltiazem caused a simultaneous depression of the rate of impulse formation in the sinus node. Diltiazem was tested in concentrations much higher than verapamil but comparable to bepridil (1 to $3 \times 10^{-6} \mathrm{M}$, chapter IV). Unlike bepridil, diltiazem did not affect conduction properties in the atrial myocardium, emphasizing the selective calcium antagonistic features of the drug. Tritthart and Koidl [269], using the isolated guinea pig sinus node and cultured sinus node cells, found a predominant effect of diltiazem on sinoatrial conduction properties and a less marked depression of the intrinsic automaticity. Kawai and colleagues [132], who tested diltiazem $2.5 \times 10^{-6} \mathrm{M}$ in the isolated rabbit sinus node, reported similar findings as obtained in the present study: a $47 \%$ reduction in sinus rate in combination with a marked depression of sino-atrial conduction. The negative dromotropic action of diltiazem in the border zone of the sinus node is in agreement with the effects on atrioventricular conduction [132,259].

Disopyramide, classified as a class I antiarrhythmic drug, is known to affect sinus rate and atrioventricular conduction in a direct manner, but also indirectly by an anti- 
cholinergic effect $[21,130,181,256,274]$. To eliminate the indirect mode of action atropine $\left(1 \times 10^{-6} \mathrm{M}\right)$ was added to the superfusion fluid. It appeared that disopyramide depresses, similar to diltiazem, both sino-atrial conduction properties and sinus automaticity in a dose-related manner. However, the negative chronotropic actions were less prominent than the influence on sino-atrial conduction. A decrease in sinus rate after addition of disopyramide was also found by others. Mirro and coworkers [181], using the isolated right atrium of the guinea-pig, found also a negative chronotropic effect of disopyramide. Similar results were obtained by Katoh et al. [130], who used the isolated rabbit sinus node. The direct depressive offect of disopyramide on sinus automaticity can be explained by a reduction of the diastolic depolarization rate and a prolongation of the action potential duration in nodal fibers $[130,274]$. However, the literature provides only limited information on the depression of sino-atrial conduction properties. Only Katoh and coworkers [430] studied this issue but did not find a significant change in sino-atrial conduction with disopyramide. This is in contrast to the findings of the present study and might be explained by the different techniques used. Katoh and colleagues used the estimated sino-atrial conduction time (SACT) and measured the latency between the moments of activations of a fiber in the pacemaking center and the crista terminalis. However, both techniques are only valid if the site of pacemaking within the sinus node remains constant, which is questionable in case of disopyramide regarding its direct actions on sinus automaticity.

The class I properties of disopyramide were illustrated by a depression in atrial conduction velocity and a prolongation of atrial refractoriness. This is in agreement with the findings of other authors $[21,123]$ and might be the underlying mechanism for the negative dromotropic effect in the sino-atrial border zone, since depolarization of these fibers partially depends on the fast sodium inward current $[145,161]$. However, this does not exclude an additional slow channel blocking action of disopyramide. In comparison to bepridil, verapamil and diltiazem, disopyramide exhibited the least potent effects on sinus function.

From the present results we concluded that verapamil, diltiazem and disopyramide are characterized by typical depressive actions on sinus function. Only verapamil resembles bepridil in its predominant effect on sino-atrial conduction, although at far lower concentrations. Diltiazem depresses sino-atrial conduction at similar concentrations as bepridil but exerts, in contrast to bepridil, an equivalent depressive action on sinus automaticity. Disopyramide depresses both sino-atrial and atrial conduction properties about equally, whereas sinus rate is reduced moderately as well. 

Chapter VI

EXTRACELLULAR MAPPING OF THE SINUS NODE REGION DURING ATRIAL FIBRILLATION 



\section{Introduction}

After the early studies on atrial fibrillation by Sir Thomas Lewis $[152,153,157,159$, 160], reentrant excitation became generally accepted as the mechanism underlying this arrhythmia $[10,167,188]$. The fibrillatory mechanism was reconsidered when several experimental studies demonstrated that atrial fibrillation could be induced by local acetylcholine or aconitine application to the atrial muscle $[182,232,234,235]$. The 'ectopic-focus' theory was introduced which explained auricular fibrillation by the rapid discharge of single or multiple automatic foci [210,233-235]. In the light of this concept several authors suggested a cruclal role of the sinus node in the genesis and perpetuation of atrial fibrillation $[192,226,235,243]$.

On the other hand, the initial circus movement theory was also strengthened by additional experimental evidence [203,219,220]. In a series of studies between 1959 and $1965 \mathrm{Moe}$ and coworkers compared atrial fibrillation induced by local aconitine application to fibrillation evoked by electrical stimulation [182-185]. Both types appeared to rely on a different fundamental mechanism. Aconitine-induced fibrillation depended on a single automatic focus located at the site of application, and was most adequately described as 'fibrillatory conduction'. In contrast, electrically induced fibrillation was not dependent on the site of induction and was indicated as 'true' atrial fibrillation. To explain the features of 'true' fibrillation Moe and Abildskov formulated the multiple wavelet theory [182,183]. In this model fibrillation is based on multiple independent activation waves wandering randomly through the atrial muscle around local areas of transient inexcitability [182-185]. Recently, Allessie and colleagues confirmed Moe's multiple wavelet theory by reconstruction of the atrial excitation sequence during electrically induced fibrillation in the dog heart [6]. In this study no evidence was obtained for rapid discharging foci or any contribution of the sinus node to the fibrillatory process. However, the last possibility could also not be excluded [6].

When multiple reentrant wavelets represent the dominant mechanism of fibrillation, the sinus node might still add to the stability of this process by increasing the average amount of wavelets. This speculation is favoured by the demonstration of sinus reciprocation and sinus node reentry [5,95]. Furthermore, in recent experimental studies it was found that suppression of the sinus node by surgical or chemical techniques reduced both the inducibility and the stability of atrial fibrillation $[13,48,187]$. In patients with sick sinus syndrome the incidence of atrial fibrillation is higher than in normal subjects $[20,76]$.

It was the aim of the present studies to investigate the possible role of the sinus node in the fibrillatory process. The findings are described in chapters $\mathrm{VI}, \mathrm{VII}$ and VIII. In the isolated rabbit heart extracellular mapping techniques were used to in- 
vestigate the fibrillatory excitation pattern in the atrium around the sinus node. The results of these experiments are described in the present chapter.

\section{Methods}

\section{The preparation and induction of atrial fibrillation}

Flemish Giant rabbits of either sex and weighing $4.5-5.0 \mathrm{~kg}$ were heparinized (2000 IU iv) and killed by cervical dislocation. After midsternal thoracotomy the heart was quickly excised. The aorta was cannulated and connected to a Langendorff perfusion system filled with warmed $\left(37^{\circ}\right.$ Celsius) Tyrode solution. The perfusion pressure was maintained at $50 \mathrm{mmHg}$ which resulted in a coronary flow of $40-50$ $\mathrm{ml} / \mathrm{min}$. The Tyrode solution was saturated with a mixture of $95 \% \mathrm{O}_{2}$ and $5 \% \mathrm{CO}_{2}$ and contained $130 \mathrm{mM} \mathrm{NaCl}, 5.6 \mathrm{mM} \mathrm{KCl}, 24.2 \mathrm{mM} \mathrm{NaHCO} 3,2.2 \mathrm{mM} \mathrm{CaCl}, 0.6 \mathrm{mM}$ $\mathrm{MgCl}_{2}, 1.2 \mathrm{mM} \mathrm{NaH}_{2} \mathrm{PO}_{4}$ and $12.2 \mathrm{mM}$ glucose. The $\mathrm{pH}$ was $7.35 \pm 0.05$.

In 9 experiments atrial fibrillation was induced by rapid pacing at Bachmann's bundle or the left atrial appendage. For stimulation a bipolar surface electrode was used (teflon-coated silverwire, $0.25 \mathrm{~mm}$ diameter) connected to a programmable stimulator (Janssen Scientific Instruments). Successive episodes of atrial fibrillation were evoked by rapid pacing during $0.5-2.0$ seconds with a frequency of 20 or 50 $\mathrm{Hz}$. The individual stimuli had a duration of $2 \mathrm{~ms}$ and an intensity of 6 times diastolic threshold. In 9 experiments the atrial spread of excitation around the sinus node was 'mapped" by simultaneous recording of 248 endocardial electrograms.

\section{Endocardial mapping of the atrial activation pattern}

A multi-electrode probe was developed for simultaneous recording of multiple endocardial electrograms from the atrium around the sinus node. A schematic drawing of the probe is given in panel A of figure 6.1. The probe was spheroidal (14 mm diameter) with two excentrically positioned cylindrical extensions. It was made of silicon (Silastic, Dow Corning) and contained 248 unipolar surface electrodes (teflon-coated stainless steel wire, $0.2 \mathrm{~mm}$ diameter), arranged in a regular configuration and covering a complete hemisphere of the probe. The interelectrode distance was $1.25 \mathrm{~mm}$. After connection of the heart to the perfusion system, an incision was made in the right ventricular free wall. Through this incision the probe was introduced into the right atrial cavity. The cylindrical extensions were positioned in the orifices of the superior and inferior caval veins. To get a reproducible position 

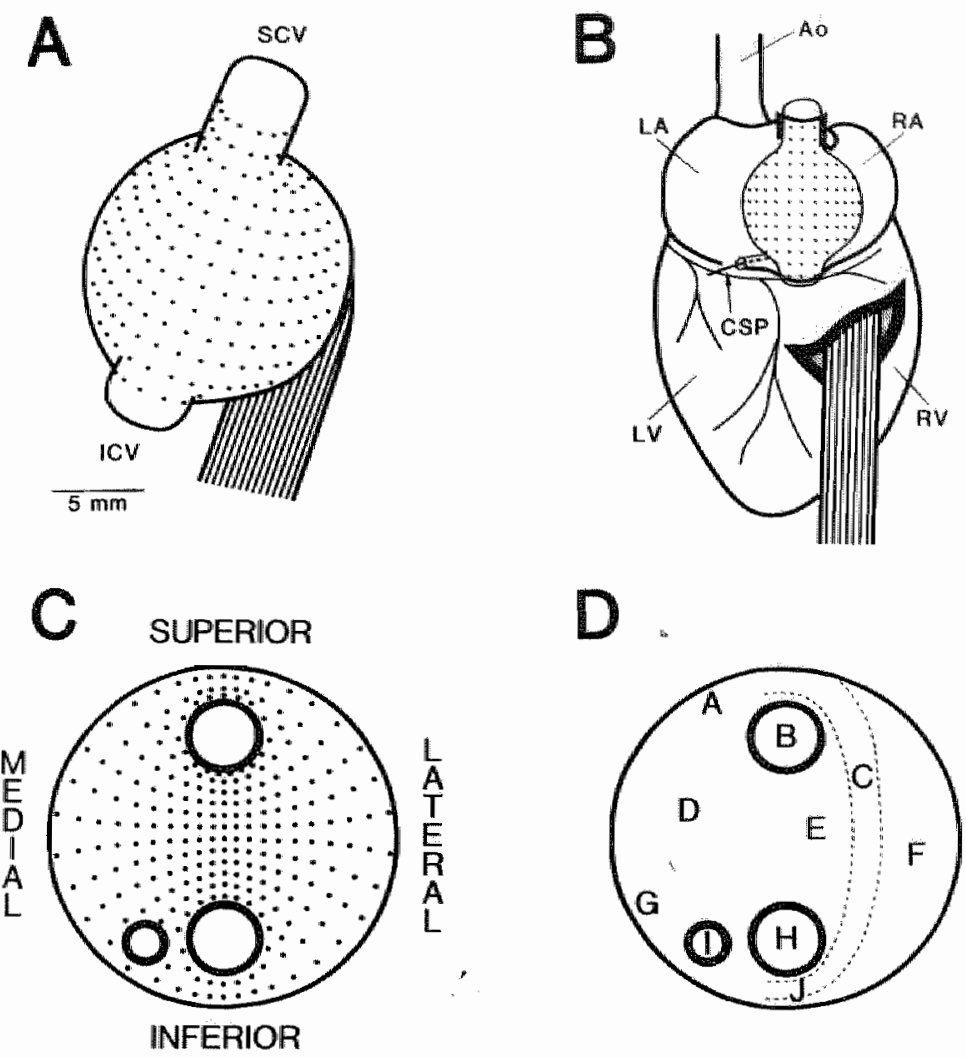

\section{Figure 6.1:}

Panel A: Schematic drawing of the multtelectrode probe. The probe is spheroidlal with two excen trical extensions. The black dots represent the lndlvidual surface electrodes (248) coverthg one hall of the sphere. At the back of the probe the electrode-leads converge into a main cable which is connected to the mapping system. SCV = superior caval vein; ICV =Inferior caval vein.

Panel B: The electrode probe in position, seen from the posterior side of the heart. Through an inclsion in the right ventricular wall the probe was introduced into the right atrlal cavity. The two axtensions were placed in the orifices of the caval veins. To ensure a flixed and reproducable position of the probe, the coronary sinus was cannulated by a metal pinlet (CSP), which fitted in a hole in the mapping electrode.

Panel C: Two-dlmenslonal projectlon of the mappling area. The electrode positions are indicated by dots. The gradual increase in interelectrode distance from the center to the periphery of the map Is a result of the two-dlmensional projection. The real interelectrode distance was $1.25 \mathrm{~mm}$ in all reglons.

Panel D: The mapping area In relation to the anatomical landmarks. A: Bachmann's bundle; $B$ :. s.u. perlior caval veln; $C$ : crlsta termilnalis; $D$ : posterlor atrial septum; $E$ : silnus node area; $F$ : posterlor lateral wall; G: AV nodal input; H: inferlor caval veln; l: coronary sinus; J: Eustachian ridge. 
of the mapping electrode relative to the geometry of the atrium the coronary sinus was epicardially cannulated by a metal pinlet which was inserted into a hole in the intracavitary probe. In panel $B$ of figure 6.1 a schematic representation of the probe in situ is given. Panel $\mathrm{C}$ shows the two-dimensional projection of this 'mapping' area, which covers the sinus node region, the caval orifices and the surrounding atrial myocardium. The black dots show the respective recording sites. In panel $D$ the anatomical landmarks in the mapping area are indicated.

The individual leads were converged into a main cable and connected to an amplification-unit containing 256 differential amplifiers. After amplification (200-300 times) and filtering of low-frequency components (less than $3 \mathrm{~Hz}$ ) the analogue signals were sampled by multiplexers ( $1 \mathrm{KHz}$ sampling rate) and converted into a single digital signal. This signal was recorded on standard video tape for off-line analysis. The electrograms were fed into a buffer memory (1 Mbyte) which was continuously updated. In this way the last 4 seconds of each signal were always available for (on-line) analysis. Up to 32 signals could be displayed on conventional cathode ray tubes. After the experiment the data were read from video tape to localize the points of interest. The buffer memory could be frozen and an analyse-window of minimally 640 and maximally $4096 \mathrm{~ms}$ could be chosen. The electrograms in this analyse-window were transferred to a personal computer (Olivetti M380) for reconstruction of the activation pattern. An algorithm automatically detected the steepest point in the negative deflections in each electrogram. These points were indicated as the moments of local activation of the tissue underneath the recording electrode and were labeled by a time-marking. The local activation times were related to an arbitrarily chosen time reference and displayed in an activation map. Within this map individual recording sites could be selected to study the morphology of the electrogram and, if necessary, the time-markings could be edited manually. Additionally, signal maps could be produced to study the spatial electrogram morphology in different regions of the mapping area. In the maps isochrones ( 5 or $10 \mathrm{~ms}$ time steps) were drawn by hand to visualize the propagation of the activation waves $[7,99]$.

In most experiments an incision in the ventricular septum was made to create total AV block to reduce the rate of ventricular complexes in the atrial electrograms. After positioning of the electrode probe a stabilization period of 30 minutes was taken into account before measurements were started. 


\section{Results}

\section{Electrical activity during sinus rhythm}

In 9 experiments the atrial spread of excitation during stable sinus rhythm was mapped. In all preparations a marked spatial variation in local electrogram morphology was found. In the upper panel of figure 6.2 a representative example is shown. The individual complexes are plotted at their respective recording sites. At the exit of the sinus node (crista terminalis) the electrograms showed large negative complexes indicating the local start of an atrial depolarization wave. In the posterior lateral wall, distally from the sinus exit, the local electrograms developed a biphasic morphology. In the posterior atrial septum the electrical complexes were also large and biphasic. Towards the lower part of the septum the electrograms gradually lost their biphasic nature to change into small positive extrinsic complexes in the area around the coronary sinus. These electrograms frequently showed small but steep intrinsic negative deflections on the downgoing part of the extrinsic waves.

In the lower part of figure 6.2 the activation map during sinus rhythm is given. The earliest site of atrial activity is indicated by an asterisk. The spread of excitation from this spot is visualized by $5 \mathrm{~ms}$-isochrones. The dashed area in the middle of the map represents the region in which only extrinsic activity was identified corresponding to the location of the sinus node. The atrial depolarization wave originated from the sinus node region and propagated preferentially in opposite directions through the crista terminalis and around the caval veins towards the atrial septum. This explains the negative potentials found in the middle portion of the crista terminalis. After $30 \mathrm{~ms}$ both activation waves fused at the area around the coronary sinus where only positive potentials were found. In the other preparations the atrial activation pattern during sinus rhythm was similar to the present example. Only the individual location of the exit site of the sinus impulse and the atrial area activated within the first $5 \mathrm{~ms}$ differed from case to case. In figure 6.3 the first $5 \mathrm{~ms}$-isochrone and the spontaneous cycle length during sinus rhythm in 9 experiments is given. The earliest atrial activity was always found along the crista terminalis. The atrial tissue activated within the first $5 \mathrm{~ms}$ varied considerably. In some experiments this area was relatively small, whereas in other preparations almost the entire crista terminalis was activated within this time period. Apparently, in the isolated perfused rabbit heart no preferential sinus exit exists. The average sinus interval of all preparations was $326 \pm 56 \mathrm{~ms}$ (mean $\pm \mathrm{SD}$ ). No correlation was found between spontaneous cycle length and site of earliest activity. 

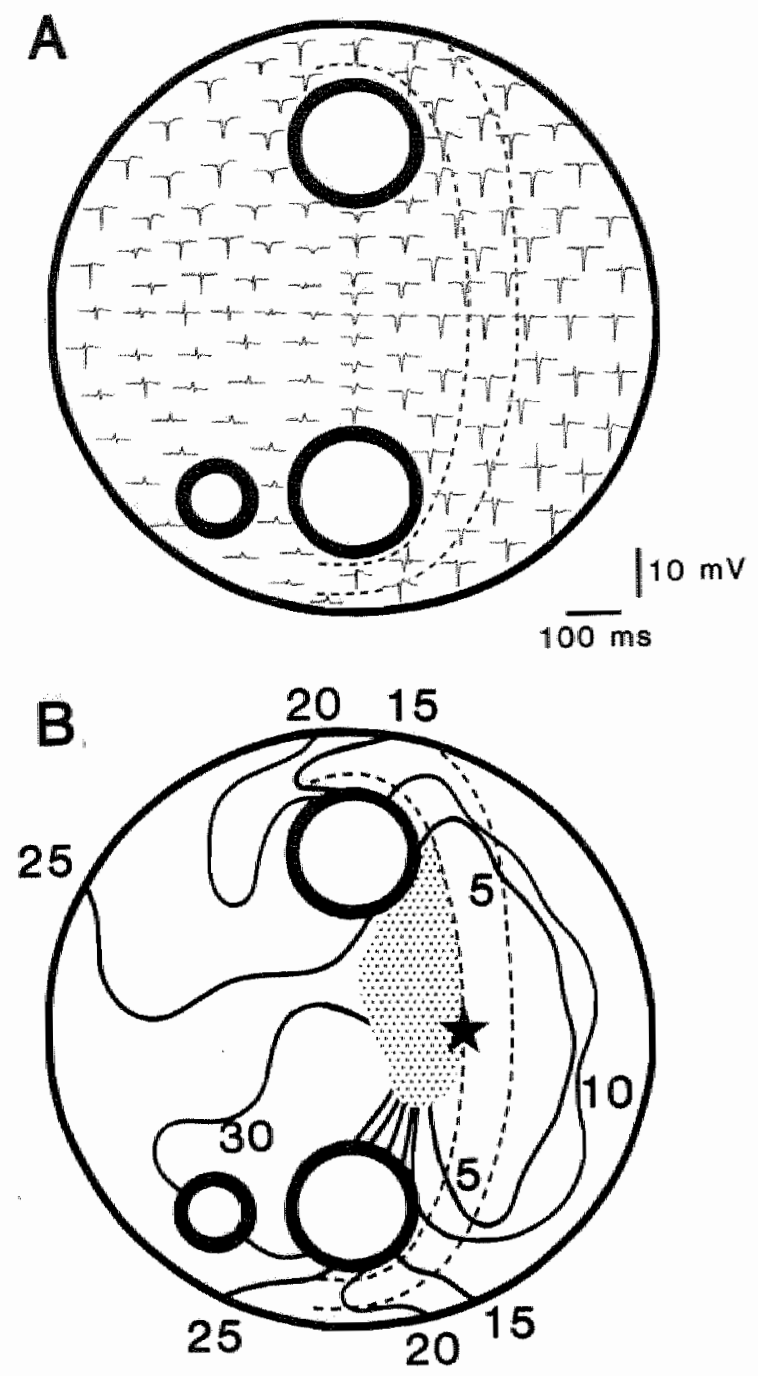

\section{Figure 6.2:}

Panel A: A selection of electrograms recarded from the sinus node area and surrounding atriall muscle durlng sinus rhythm. The electrical complexes showed a spatial varlation in amplitude and morphology. At the site of earllest atrlal activation in the crista terminalls the electrograms showed a negatlve monophasic morphology indicating local impulse origin.

Panel B: Activation map during sinus thythm. The dashed area represents the sinus node region where no Intrinsic electrical activity was recorded. The earliest negativity appeared at the crista terminalls (asterisk). From this site of sinus exit the impulse traveled through the anterlor and posterlor internodal pathway towards the atrial septum. The sequence of activation is vizualised by isochrones ( $5 \mathrm{~ms}$ time resolution). The AV nodal input region was activated after $30 \mathrm{~ms}$. 


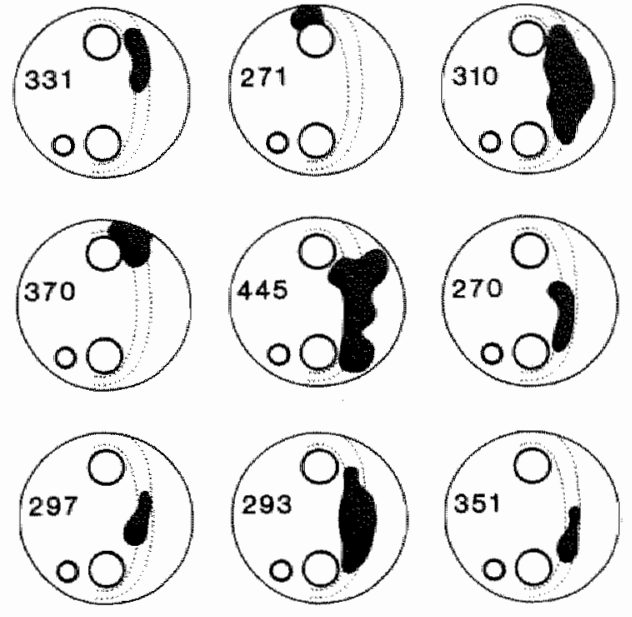

Figure 6.3:

The earliest atrial activation during sinus rtythm in all preparations. The black areas lepresent the first $5 \mathrm{~ms}-1 \mathrm{so}$ chrone. The site of sinus exit was always found at the crista terminalls. The area activated within the first $5 \mathrm{~ms}$ varled considerably. The concommitamt sinus Interval is given in each map. No correlation botween site of sinus exit and sinus rate was found.

\section{Local electrical activity during atrial fibrillation}

Short periods of atrial fibrillation could be induced by rapid electrical stimulation. In $27 \%$ of the attempts burst pacing resulted in an episode of fibrillation with a duration of at least 1 second. The average duration of all episodes was 2.1 seconds with a maximum of 7.2 seconds $(n=23 i)$. Figure 6.4 shows 19 electrograms, recorded during atrial fibrillation from the atrium around the sinus node (upper panel). The configuration and amplitude of the individual complexes varied considerably. The electrograms demonstrated a clear temporal and spatial variation in cycle length. The amplitude and slope of the complexes varied markedly between different regions. The signals from the crista terminalis and lateral wall showed mainly steep deflections of a high amplitude. In the atrial septum the electrograms generally were lower in amplitude but still revealed steep negative deflections representing local activity. In the region superior to the orifices of the coronary sinus and inferior caval vein the electrograms showed mainly extrinsic potentials, indicating an area with only an occasional activation. In figure 6.5 three atrial electrograms are shown in greater detail. The upper electrogram was obtained from the atrial septum, the middle and lower tracing from the superior and inferior lateral wall respectively. Within the same signal rapid deflections of high amplitude could suddenly change into low amplitude and multiple component complexes. The successive activation cycles varied markedly. This chaotic appearance is typical for atrial fibrillation [6]. 

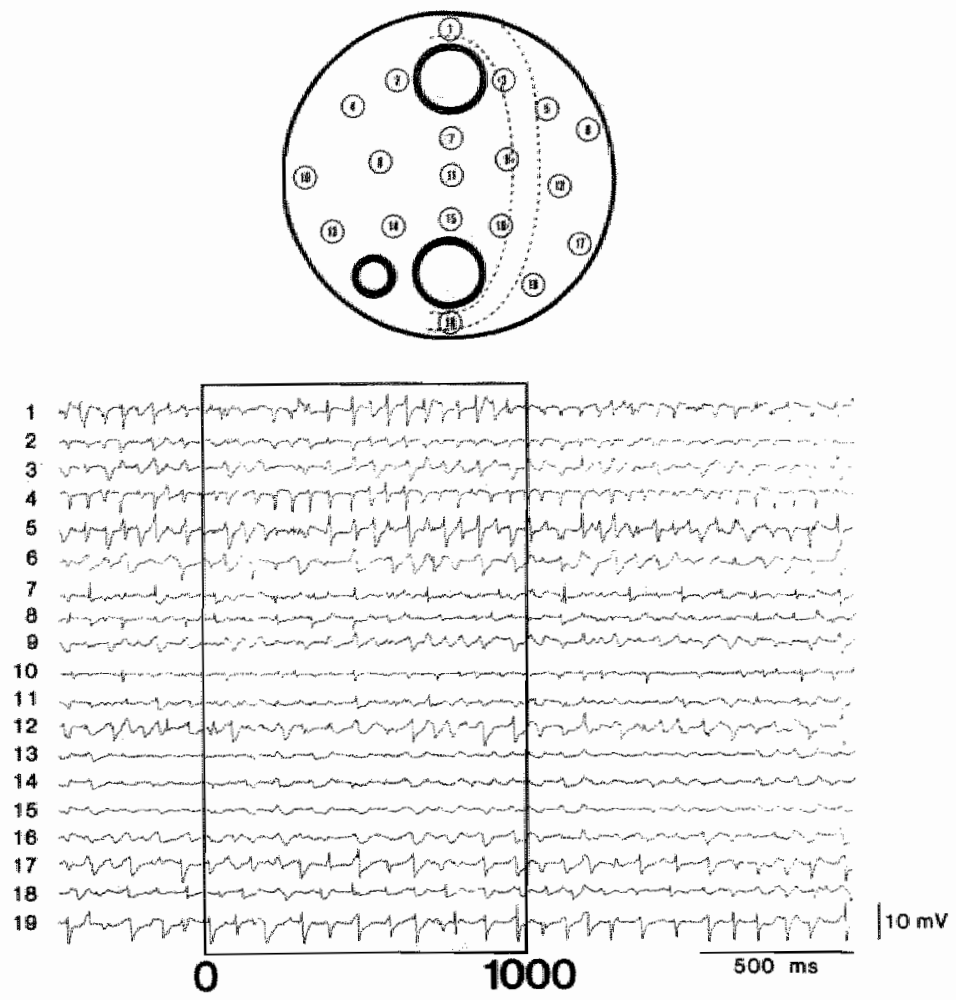

\section{Figure 6.4:}

A selection of 19 unipolar electrograms recorded simultaneously from the sinus node region and surrounding atrium during atrial fibrillatlon. The Individual recording sites are indicated in the map at the top of the flgure. The electrograms demonstrated a great temporal and spatial variation in morphology and activation cycle which is typlcal for fibriliation. The frame indicates a time period of 1 sacond of which the activation maps are shown in figure 6.6.

\section{Atrial activation pattern during fibrillation}

In 9 preparations the atrial spread of excitation during fibrillation was reconstructed. The activation maps revealed that the sinus node area was constantly surrounded by one or more wavelets coming from different directions. In figure 6.6 the fibrillatory waves are reconstructed during a 1 second period of atrial fibrillation. In each map the pathway of the individual wavelets is indicated by arrows. The shaded areas represent tissue in which no electrical activity was recorded during the respective time frames. If the wavelet was blocked because of local refractoriness of the atrial myocardium this is indicated by a block-sign (-11). 


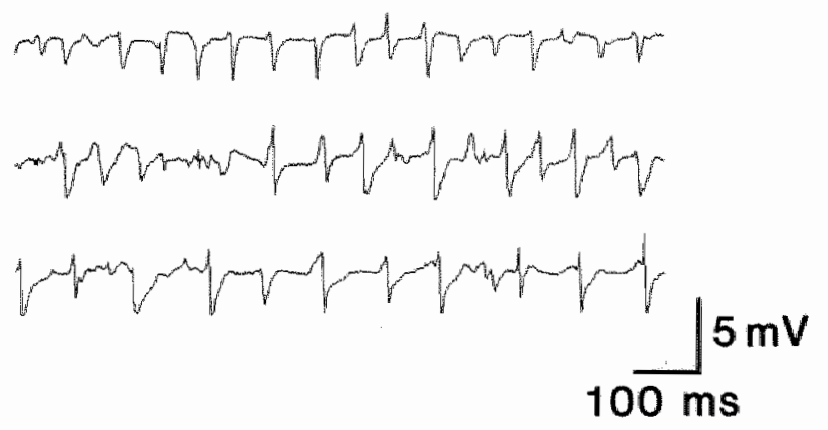

Figure 6.5:

Three unlpolar electrograms recorded simultaneously at the atrial septum (upper tracing); the supertor (middle tracing) and inferior flower tracing) lateral wall during atrial fibrillation. The successive complexes in each recording varied markedly in amplitude, steepness, morphology and cycle length.

The first map (A) starts at an arbitrary moment $t=0$ when an impulse invades the sinus node region from the lateral wall, followed after $10 \mathrm{~ms}$ by a second wavelet coming from the superior septum. At $40 \mathrm{~ms}$ (B) an impulse enters the mapping area from the superior crista terminalis and propagates downward in the lateral wall. in the region of the inferior caval vein this impulse describes a clockwise reentry (maps $C$, D and E) while a new wavelet entered from the superior septum at $100 \mathrm{~ms}(C)$. A branch of the reentrant circuit travels upward and around the superior caval vein to the atrial septum where it collides at the Eustachian ridge on its complementary wave from the lateral wall $(F)$. A single fibrillatory impulse thus has propagated during $230 \mathrm{~ms}$ (maps $B$ to $F$ ) around the sinus node region. At time moments 270 and 320 ( $G$ and $H$ ) new impulses invade the sinus node region from the superior septum. In map I, at $380 \mathrm{~ms}$, two wavelets appear, one at the superior septum and the other at the superior lateral wall. Then, starting from time $=410(\mathrm{~J})$, the area covered by the mapping electrode is electrically silent during a time period of $50 \mathrm{~ms}$. At moment $460(\mathrm{~K})$, two new wavelets simultaneously enter the map, one impulse coming from the superior septum and the other from the AV junctional region. Both activate the entire atrium around the sinus node. In the subsequent maps ( $L, M, N$ and O) fibrillatory wavelets appear in the atrial septum at 520,630 and $680 \mathrm{~ms}$ and activate the lateral wall. In map P the latter impulse describes a clockwise reentry in the inferior lateral wail before it leaves the mapping area. Meanwhile two impulses entered the map at moment 730 from the atrial septum and superior crista terminalis. In map Q two wavelets appear at $780 \mathrm{~ms}$ at the superior septum and lateral wall, followed at moment 850 by a next impulse from the superior septum which describes a counter-clockwise reentrant loop in the upper lateral wall (continued in map $S)$. This reentry is partially situated outside of the mapping area ( $R$ and $S$ ). At 

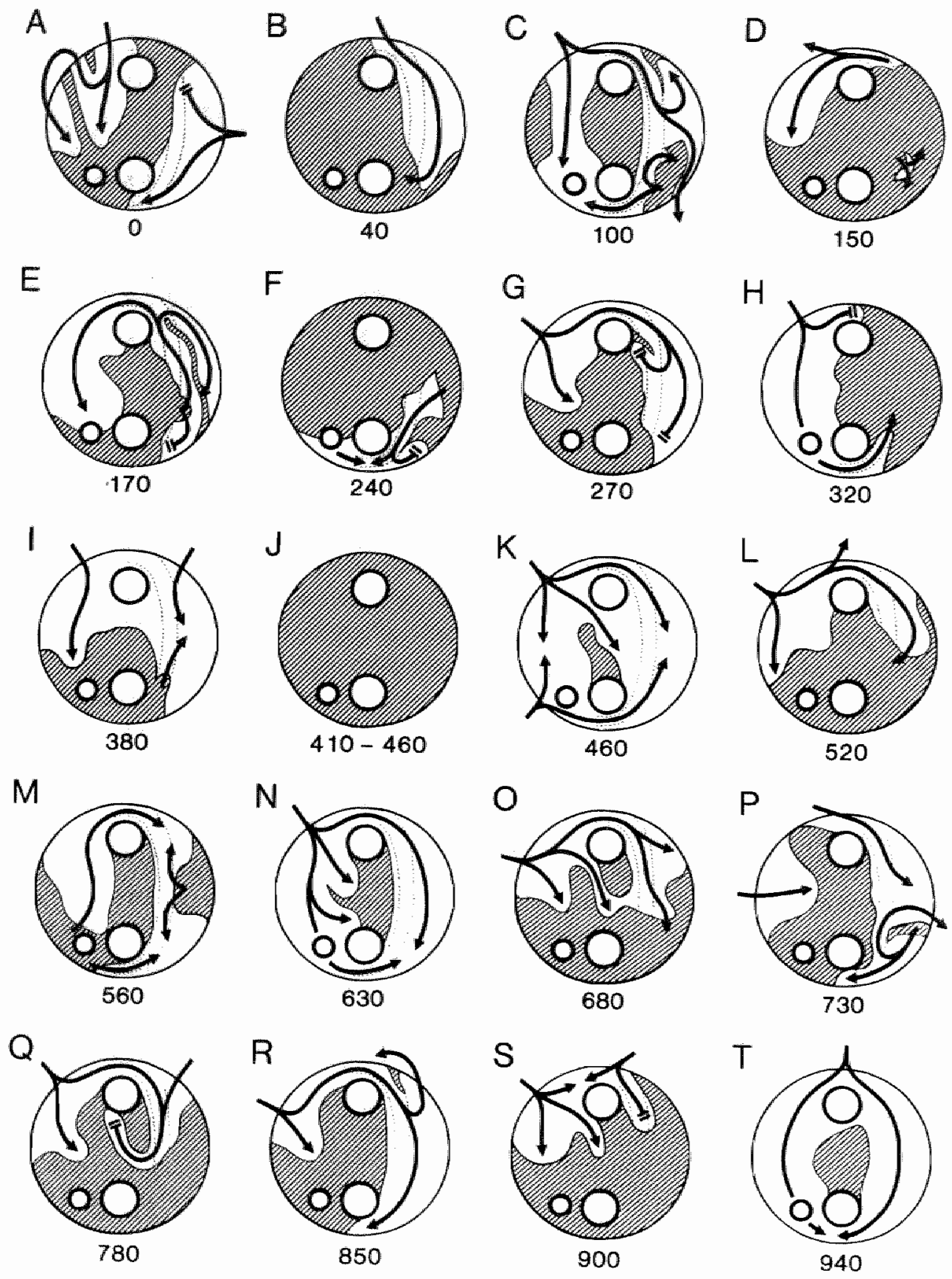
time 900 (S) and $940(T)$ impulses enter from the superior septum and superior crista terminalis.

The maps in figure 6.6 demonstrate that electrically induced atrial fibrillation in the perfused rabbit heart is based on multiple activation waves propagating through the atrial myocardium. These wavelets enter the atrium around the sinus node from different directions although the region of the superior atrial septum functions as a major input. About $60 \%$ of the fibrillatory impulses entered the sinus node area from this region. During fibrillation no extracellular activity was recorded in the intercaval area neighbouring the inferior caval vein. Sometimes impulses transferred through the intercaval area between septum and lateral wall (map K).

\section{Activation waves arising from the sinus node region during atrial fibrillation}

The activation maps during atrial fibrillation sometimes revealed an excitation wave which seemed to originate from the sinus node region. In figure 6.7 an example of this phenomenon is shown. The 9 successive maps (panels A to I) cover a total time period of $550 \mathrm{~ms}$ and show the pathway of the various fibrillation waves by arrows. An electrogram from the crista terminalis is depicted at the top of the figure. As described above, during atrial fibrillation the sinus node was continuously invaded by impulses coming from different directions. The map in the center of the figure (panel E), however, shows a new impulse arising at the upper part of the crista terminalis (asterisk). This depolarization wave could not be explained by propagation of one of the preceding atrial wavelets. Both the site of origin of this new wavelet and the fact that the local electrograms showed negative monophasic complexes strongly suggests that it originated from the sinus node. From the site of its presumed sinus exit the new impulse participated in the fibrillatory process. It was blacked towards the superior lateral wall but propagated in opposite directions through the crista terminalis and activated the atrial septum and inferior lateral wall.

\section{< Figure 6.6:}

The atrial spread of excitation during one second of atrial fibrillation as indicated in figure 6.4. Time moment zero was arbitrarlly chosen. The arrows in each frame deplat the propagation of subsequent fibrillatory wavelets in the atrium around the sinus node. When an activation wave extinghuished because of local refractoriness this is indlcated by a block sign ( -11 ). The begin time of the successive timeframes is indicated below each map. The shaded areas represent tissue in which no electrical actlvity was recorded during the respectlve time frame. During atrial fibrillatlon the sinus node region was continuously bombarded by flbrillatory wavelets coming from different directions. 

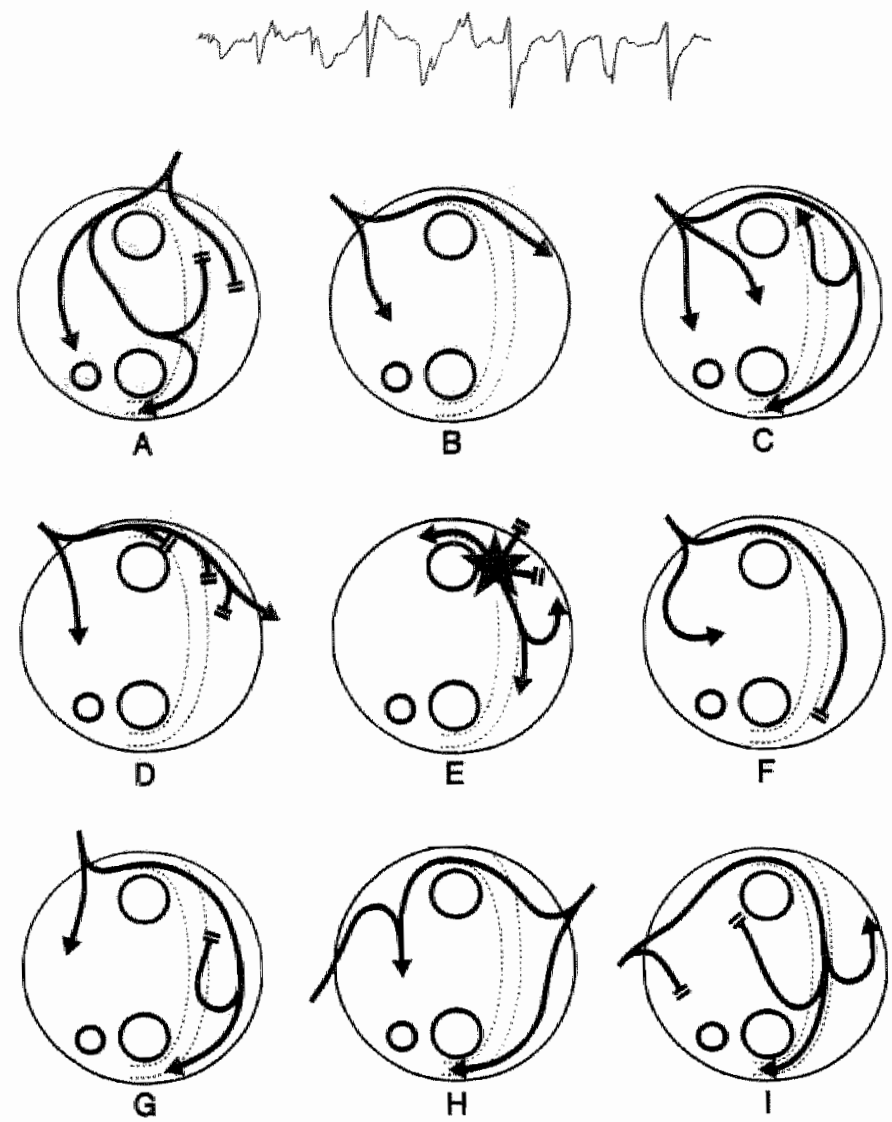

\section{Figure 6.7:}

Originatlon of a sinus Impulse during atrial fibrillation. The maps ( $A$ to $)$ show the propagation of the successive fibrlllatory impulses during a time perlod of $550 \mathrm{~ms}$ represented by the electrogram at the top. In map $\mathrm{E}$ an atrial wavelet arises from the sinus nodie at the upper crlsta terminalis and particlpates in the fibrillatory process (asterisk).

In the present experiments the atrial activation pattern of 5 longer episodes (2$6 \mathrm{~s}$ ) of fibrillation was reconstructed with a total duration of 15.4 seconds. From these series we found that on the average about 1 out of each 10-12 fibrillation waves originated from the sinus node. The point of sinus exit varied considerably. In figure 6.8 the points of origin of fibrillatory waves emerging from the sinus node are indicated by asterisks. It appeared that the atrial wavefronts could originate along the upper and middle part of the crista terminalis, but also at the septal side 


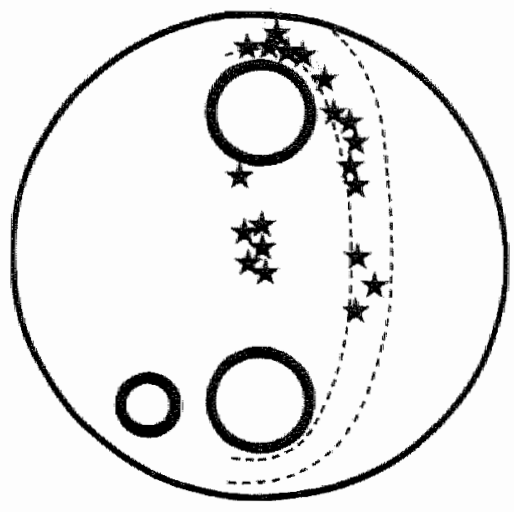

Figure 6.8:

The vartous sites of sinus impulses durIng atrial fibrillation as identifled from analysis of several long epilsodes of atrial fibrillation. The orlgination of impulses from the sinus node reglon was not $\mathrm{re}$. lated to a preferential exit point but could occur all around the sinus node.

of the sinus region. This indicates that the spontaneous manifestation of fibrillation waves is not related to a preferential site of exit but might occur anywhere around the sinus node area.

\section{Discussion}

In the present study we focussed on the question whether and how the sinus node influences the process of atrial fibrillation. The choice of the preparation was determined by two crucial prerequisites. The atrial muscle had to be large enough to fibrillate [182] and the sinus node region should be accessible for exploration with microelectrodes (chapter VII). Preliminary experiments revealed that the perfused heart of large rabbits fulfilled these prerequisites.

\section{Sinus rhythm}

With the extracellular recording techniques used in the present experiments the electrical activity of the sinus node could not be detected. As a consequence, the exact origin of sinus impulses could not be identified with our extracellular mapping techniques. Instead, the earliest electrical activity found in the present maps represents the site of exit of the sinus impulse to the atrium. This invariably occurred at the crista terminalis which is in agreement with the findings of others in isolated sinus node preparations $[23,172,205,225]$. The local electrograms at the sinus node exit showed a primarily negative morphology. This points to focal manifestation of a depolarization front as demonstrated by Masuda and Paes de Carvalho [172]. In the 
isolated rabbit sinus node these authors systematically identified small deflections in unipolar surface electrograms from the crista terminalis and attributed these potentials to local sino-atrial conduction. We could not record these potentials with the presently used recording techniques.

The site of sinus exit was not related to a specific location. Instead, it could be fourid along the entire crista terminalis. From the atrial exit site the sinus impulse activated the lateral atrial wall and propagated in opposite directions through the crista terminalis. Via the anterior (Bachmann's bundle) and posterior route (Eustachian ridge) both sinus wavefronts activated the atrial septum and reached almost simultaneously the AV nodal input. These results confirm the earlier findings of Sano and Yamagishi [225] and Hiraoka and Adaniya [98] who studied the right atrial activation sequence in the isolated right atrium of the rabbit heart.

\section{The influence of the sinus node on atrial fibrillation}

During short episodes of electrically induced atrial fibrillation the unipolar surface electrograms revealed a typical temporal and spatial variation $[6,182]$. Reconstruction of the atrial activation sequence demonstrated multiple independent wavelets propagating randomly through the atrial muscle. Local areas of refractoriness created local areas of unidirectional conduction block which sometimes resulted in functional reentry [4]. Although we did not map the entire atrium, this is in agreement with the findings of Allessie et al. [6] in the dog heart and confirms Moe's multiple wavelet theory for the rabbit atrium $[182,183]$.

The surface electrograms recorded during fibrillation from the sinus node region and the area superior of the coronary sinus mostly did not show clear intrinsic deflections. However, a small functional pathway between the atrial septum and lateral wall was present from which small but steep intrinsic deflections were registered. This is in agreement to the findings of Satake et al. [231] and Hiraoka and Adaniya [98] who demonstrated direct impulse conduction between the crista terminalis and atrial septum after obstruction of the anterior and posterior internodal pathway. In the present experiments incidentally a fibrillation wave propagated through this intermediate route.

From the present experiments it appeared that during atrial fibrillation the sinus node is constantly approached by multiple atrial wavelets. These wavelets enter the atrium around the sinus node from different directions. The most frequent site of entrance was the superior septum which is contiguous with the bundle of Bachmann. Since this bundle is the major connection between the left and right atrium, the left atrium is a major source of input. 
An important observation was that some fibrillation waves seemed to originate from the sinus node region. With an average incidence of about once per second a new impulse emerged from the sino-atrial junction to participate in the fibrillatory process. The point of exit coincided with those during sinus rhythm but could also be located at the septal side of the sinus node. A sino-septal exit during sinus rhythm was not found in the present experiments although functional sino-septal conduction was present as demonstrated by others $[98,231]$. Regardless of the underlying mechanism, impulses originating from the sinus node during atrial fibrillation will participate in perpetuation of the arrhythmia by increasing the number of atrial wavelets. In addition, early sinus impulses might also be of importance in immediate restarting the fibrillatory process in case of spontaneous termination $[6,53$, $137,226]$.

The results of the present experiments suggest that the sinus node may function as a source of impulses during atrial fibrillation. This might explain the findings of earlier experimental studies in which facilitation of atrial fibrillation by the sinus node was suggested. Scherf et al. [235] showed that acetylcholine-induced atrial fibrillation in dogs could be terminated by simultaneous cooling of the sinus node and the AV node. Nadeau and coworkers [187] induced episodes of atrial fibrillation in dogs after local acetylcholine application on the right atrial surface followed by mechanical stimulation. After suppression of the sinus node by injection of alcohol into the sinus node artery both the inducibility and the duration of atrial fibrillation were drastically reduced. Azuma et al. [13] found that aconitine induced atrial fibrillation and flutter in dogs was markedly reduced in incidence and duration after chemical or mechanical destruction of the sinus node. 



\section{Chapter VII}

INTRACELLULAR

RECORDINGS FROM THE SINUS NODE DURING ATRIAL FIBRILLATION 



\section{Introduction}

Atrial fibrillation in the perfused rabbit heart is primarily based on multiple wavelets propagating randomly through the atrial myocardium (Chapter VI). To investigate the role of the sinus node in this process the posterior portion of the right atrium was mapped. This revealed that the sinus node is continuously invaded by fibrillatory wavelets coming from different directions. Sometimes however, a new impulse originated from the sinus node area and participated in the fibrillatory process. This phenomenon increased the number of wavelets and thus facilitated the perpetuation of atrial fibrillation.

The extracellular mapping technique however did not allow detailed analysis of the electrical activity in the sinus node itself. Therefore, in a subsequent series of experiments intracellular recordings were made from sinus node fibers during atrial fibrillation. The results of these experiments are described in the present chapter.

\section{Methods}

In 7 experiments the hearts of Flemish Giant rabbits were isolated and perfused as described in the 'Methods' section of chapter VI. The preparation was modified to enable endocardial microelectrode reçordings from the sinus node. After ligation of the main descending coronary arteries both ventricles were removed by a circular incision parallel to the $\mathrm{AV}$ groove. The remaining perfused atrial preparation was turned upside down and placed in a tissue-bath, the tricuspid ring being spread out by needles. Through this opening the sinus node region was visualized and fixed in a horizontal plane to an underlying silicon bar. Standard microelectrode techniques (glass capillaries filled with $3 \mathrm{M} \mathrm{KCl}$ and a tip resistance of 10-30 MOhm) were used to record intracellular potentials from fibers in different regions of the sinus node. A single roving microelectrode was used which could be accurately moved around by two vernier scales $(0.01 \mathrm{~mm})$. A dissecting microscope (magnification factor: $16 \mathrm{x}$ ) was used to determine the morphological boundaries of the sinus node area and to correlate the $x-y$ coordinates of the microelectrode position to the anatomical landmarks of the sinus node region. Atrial fibrillation was induced by rapid pacing at Bachmann's bundle or the left atrial appendage (see also Chapter VI). To obtain stable intracellular recordings the duration of the episodes of fibrillation was prolonged by lowering the potassium concentration of the perfusion fluid from 5.6 to $2.2 \mathrm{mM}$. 


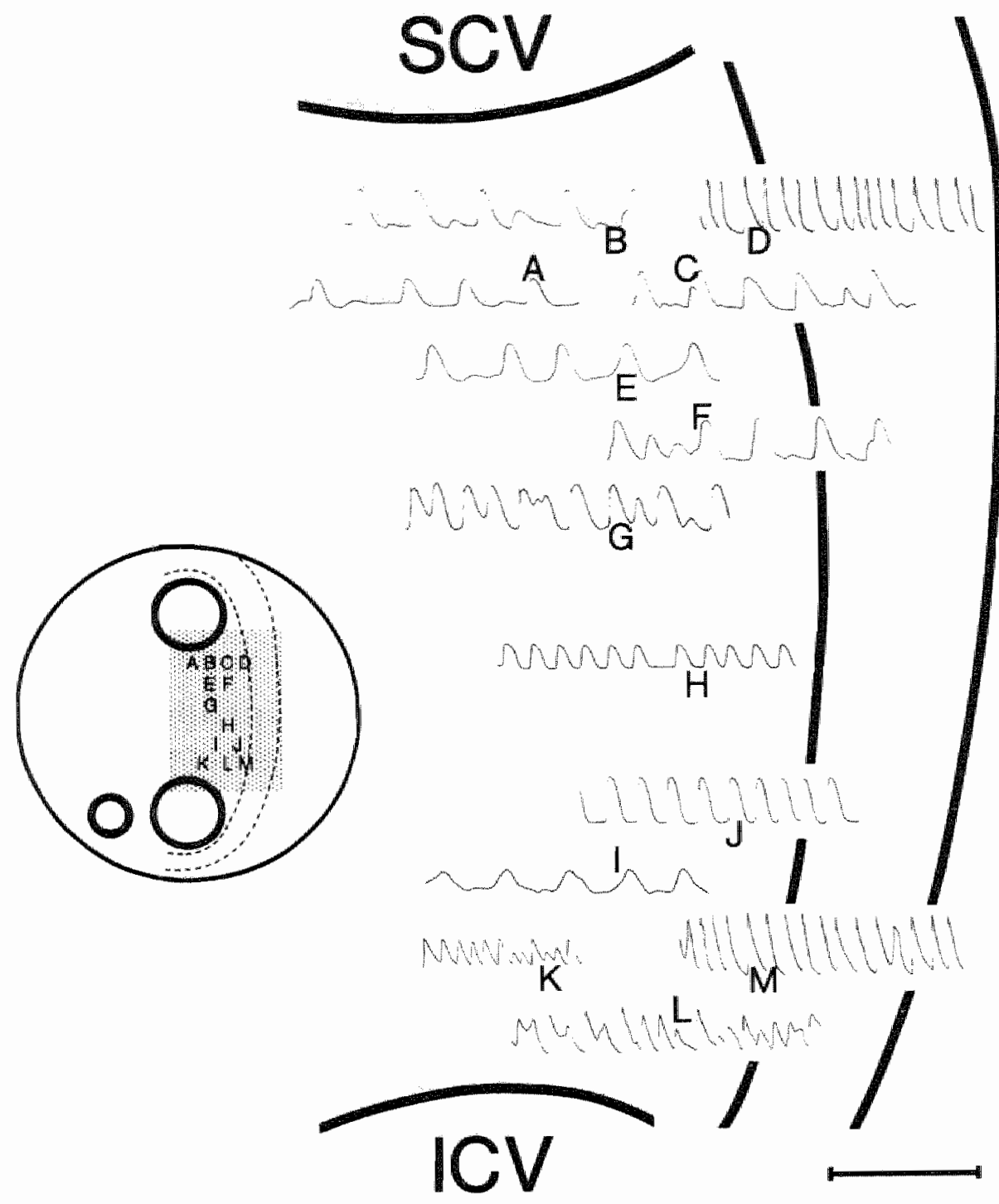

Figure 7.1:

Transmembrane potentlals recorded at several locations in the sinus node during atrial fibrillation. The dashedl area in the overall scheme (left) shows the area of interest and the sites of recording. A heterogeneous pattern of cellular activity was found. Some fibers showed a high fibrillation rate whereas others demonstrated a relatively low rate. The calibration bar at the bottom represents $1 \mathrm{~s}$ and $1 \mathrm{~mm}$. SCV = superior caval vein; $1 \mathrm{CV}=$ inferior caval wein. 


\section{Results}

\section{Sinus node action potentials during atrial fibrillation}

During atrial fibrillation multiple transmembrane recordings were made in different regions of the sinus node. A marked spatial and temporal variation in cellular activity was found. In figure 7.1 a number of intracellular potentials is shown as obtained in one of the experiments. The individual signals are plotted at the site of recording ( $A$ to $M$ ). A heterogeneous pattern of cellular activity was found. At the margin of the crista terminalis (tracings $D$ and $M$ ) the fibers showed a rapid and irregular activation rhythm, similar to the fibrillation rhythm in the atrium. In the border zone of the sinus node the cellular activation rate was clearly lower than in the atrium. Some fibers in the border zone showed a high incidence of electrotonic potentials (recordings $C, F$, and $G$ ) whereas others did not (tracings $H$ and $J$ ). The fibrillation rate in the central portion of the sinus node (locations $A, B$, and $E$ ) was again lower than in the border zone and even close to normal sinus rate. This gradual reduction in fibrillation rate from the atrium towards the center of the sinus node indicates a high degree of sino-atrial conduction block.

\section{Sino-atrial block during fibrillation}

To study sino-atrial conduction block during atrial fibrillation a series of intracellular recordings were made along a line perpendicular to the crista terminalis. The distance between the successive impalements was $0.2 \mathrm{~mm}$. In figure 7.2 the results of one of the experiments is shown. The sites of intracellular recording are indicated in the scheme at the top of the figure. Below, a bipolar surface electrogram from the crista terminalis (CT) and the successively recorded intracellular potentials at sites A to $I$ are given. Next to the different tracings the average fibrillation interval is indicated. The average fibrillation interval in the crista terminalis was $79 \mathrm{~ms}$. The first transmembrane recording (A) was obtained from a fiber at the margin of the crista terminalis and showed the characteristics of an atrial myocardial cell. The activation rhythm resembled the fibrillatory activity in the surface electrogram (CT), but the average cycle length was slightly longer $(92 \mathrm{~ms})$. Recordings $B$ and $C$ were obtained from fibers in the border zone of the sinus node at a distance of 0.2 and $0.4 \mathrm{~mm}$ from fiber $\mathrm{A}$. in these fibers the successive action potentials varied widely, both in amplitude, rate of rise and duration. On the average the action potential duration was longer than in fiber A. Fiber B showed an average cycle length of $164 \mathrm{~ms}$ and 


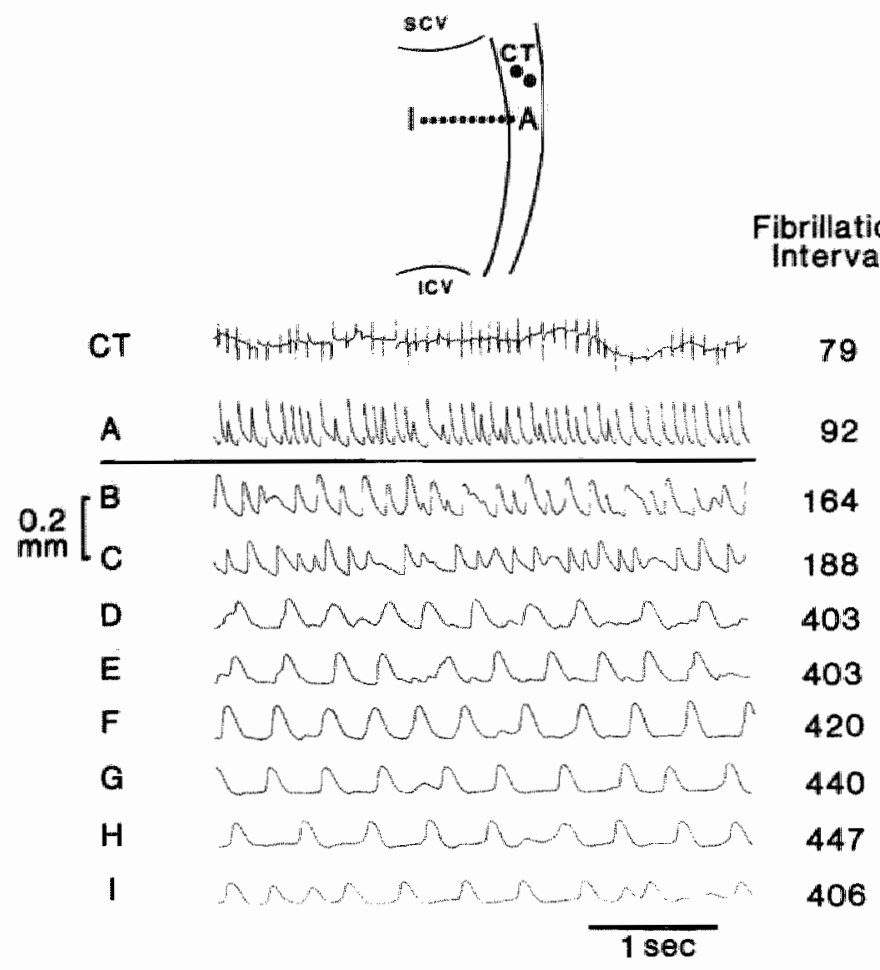

\section{Figure 7.2:}

Quantification of local fibrillation intervals in relation to sino-atrial distance. A series of intracellular recordings were made along a line perpendicular to the crista terminalis (upper scheme). A bipolar reference electrogram was recorded from the crista terminalis (CT). Tracing A was obtained from an atrlal flber al the transition between crista terminalls and border zone of the sinus node. Fecordings $B$ to I were made at regular increasing $(0.2 \mathrm{~mm})$ sino-atrial distances. The average fibrillation interval ls given at the right of each recording. This interval decreased as a function of dlstance to the atrlum. On the average less than one out of five atrlal activation waves reached the pacemaker cells in the central portion of the sinus node.

fiber $\mathrm{C}$ of $188 \mathrm{~ms}$, being about twice the cycle length found at location A. Apparently, during atrial fibrillation an average 2:1 sino-atrial entrance block existed between the crista terminalis and the border zone of the sinus node. At location $D, 0.6 \mathrm{~mm}$ apart from site $A$, a second drastical reduction of the local fibrillation rate was found. Here, the average fibrillation interval was as long as $403 \mathrm{~ms}$, being more than twice the interval at site $\mathrm{C}$, and about five times the fibrillation interval in the atrium. The fibers in locations $E$ to I showed action potentials with a slow upstroke and a long duration as usually found in the center of the sinus node. The fibrillation rate in this area was only slightly longer than in site $D$. Thus, during atrial fibrillation the pace- 
maker fibers in the center of the sinus node showed a rate of less than $20 \%$ of the atrial fibrillation rate. In the other experiments these findings were confirmed.

In figure 7.3 electrotonic modulation of the transmembrane potentials in the sinus node during atrial fibrillation is illustrated. The fibers were located at a distance of $0.0,0.2,0.6$, and $1.2 \mathrm{~mm}$ from the crista terminalis. In the atrium (upper tracing) low amplitude potentials of brief duration (triangles) were frequently interposed between well developed atrial action potentials. These potentials always occurred during the late repolarization phase of the preceding action potential and represent local intra-atrial conduction block [230]. In the second tracing, recorded from the border zone of the sinus node, similar responses were found pointing to conduction block in the border zone. Here electrotonic potentials sometimes occurred early in the repolarization phase of the preceding action potential resulting in a marked electrotonic lengthening of the action potential. This phenomenon can be seen in the middle of the signal: two successive electrotonic potentials resulted in prolongation of the duration of the action potential to more than twice its original value. At the transition between the border zone and the center of the sinus node (third tracing) the electrotonic potentials showed a lower amplitude. In this region of the sinus node the fibrillation rate was much lower than in the border zone. Between two successive action potentials often multiple electrotonic potentials were observed. The electrotonic potentials indicate that also in this region of the sinus node local con-

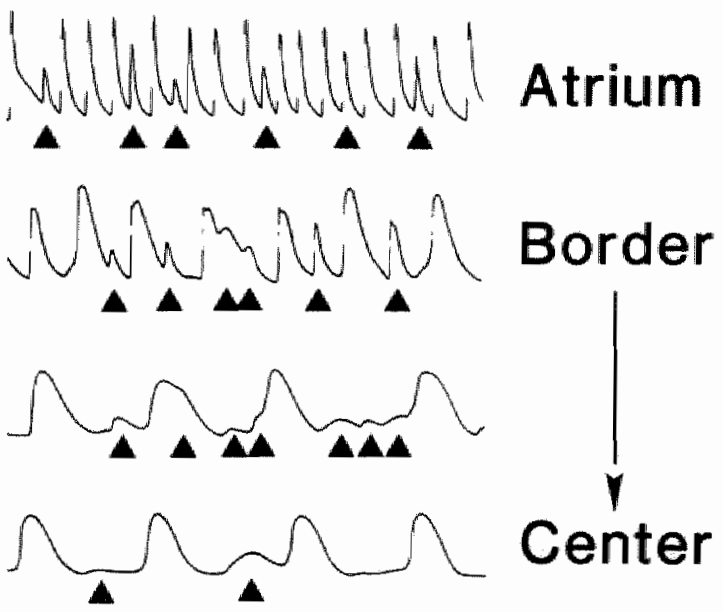

Figure 7.3:

Cellular responses and electrotonlc potentlals in the sinus node as a result of local conduction block. The upper tracing repre sents an atrial fiber at the sino-atrial transittion. Among well developed action potentials brief rem sponses of reduced amplitude and duration (trlangles) Indicate the occurrence of local conduction block. Slmilar responses were found in the border zone (second tracing). At the transition between the border zone and center of the sinus node (third tracing) the shape of these electrotonic potentials changed. In the nodal center the incidence of electrotanic responses decreased (lower tracing). 
duction block occurred. In the center of the sinus node (lower tracing) the incidence of electrotonic potentiais was reduced and rarely affected action potential configuration.

\section{Protection of the sinus node and concealed automaticity}

As described in chapter III, the maximal activation rate of fibers in the sinus node gradually decreases from the atrial fibers in the crista terminalis to the pacemaker cells in the center of the sinus node. We evaluated the relationship between this intrinsic property and the average activation rate during atrial fibrillation. In figure 7.4 the minimal pacing interval, the average fibrillation interval, and the spontaneous sinus interval are plotted as function of the sino-atrial distance. During atrial fibrillation the average activation cycle at the margin of the crista terminalis and in the sinoatrial border zone differed only slightly from the minimal pacing interval. This indi-

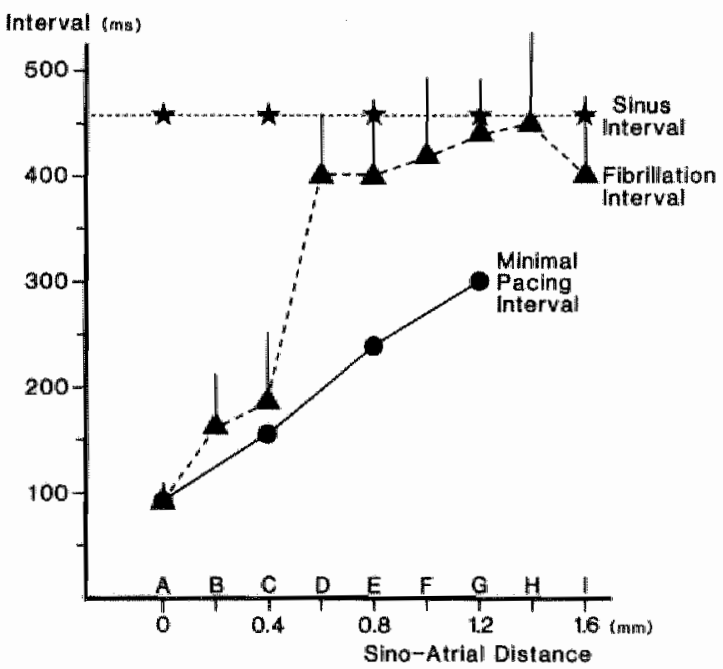

Figure 7.4:

The minimal pacing interval (closed clrcles), the mean local fibrillation interval (triangles) and the sponteneous slnus Interval (stars) as function of the sino-atrlal distance (absclssa). Sites A to I correspond to the sites of recording in figure 7.2. The variation in fibrillation Intervals is indicated by the standard deviation. At short sino-atrial distances the mean fibrillation interval was only slightly longer than the minimal Intenval during regular pacing. This was in contrast to the response in the center of the sinus node where the fibrillation interval was considerably longer than the local minimal pacing interval. Here the rate of discharge of pacemaker tibers during atrial fibrillation was almost simllar to the cycle length during normall sinus thythm. Individual fibrillation cycles often even exceeded the spontaneous sinus Interval. 
cates that the physiological prolongation of refractoriness in the border zone is a primary determinant of the local fibrillation rate and the direct cause of sino-atrial entrance block. However, at distances greater than $0.5 \mathrm{~mm}$ from the atrium this close relationship between average fibrillation interval and minimal pacing interval no longer existed. In the center of the sinus node the degree of sino-atrial conduction block during fibrillation was much higher than could be explained on basis of the longer refractory periods. In fact, during atrial fibrillation the average cycle length in the central nodal pacemaker fibers differed only slightly from the spontaneous cycle length during normal sinus rhythm. Due to the marked variation in sinus node cycle length during fibrillation, the time interval between successive action potentials frequently even exceeded the intrinsic cycle length of sinus node automaticity.

The sino-atrial border zone thus protects the pacemaker fibers in the center of the node. The majority of fibrillatory impulses was blocked in the border zone before they could reach the pacemaker area. As a consequence, fibers in the center of the sinus node often exhibited spontaneous diastolic depolarization. Occasionally, this resulted in spontaneous action potential generation in the sinus node during atrial fibrillation. In figure 7.5 an example of this phenomenon is given. The upper tracing shows a bipolar atrial electrogram during atrial fibrillation. This was recorded simultaneously with an intracellular recording from a fiber in the center of the sinus node at a distance of $1.5 \mathrm{~mm}$ from the atrium (lower tracing). The high degree of

\section{SA Entrance Block during AFib}

Atrium

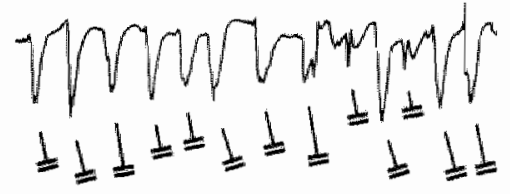

Sinus Node

Figure 7.5:

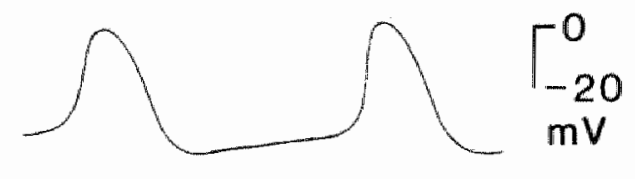

$100 \mathrm{~ms}$

Concealed automaticity in the sinus node due to sino-atrlal entrance block during atrial fibrillation. The upper signal represents a bipolar electrogram from the crista terminalls. The lower tracing depicts an Intracellular recording from a pacemaker fiber at $1.5 \mathrm{~mm}$ distance from the crista termlnalls. The presence of a high degree of sino-atrial entrance block allowed the pacemaker fiber to develop spontaneous diastolic depolarization resulting in spontaneous dlscharge of the fiber. 
sino-atrial entrance block temporarily prevented the pacemaker fiber from being activated by any of the atrial fibrillatory impulses and the pacemaker fiber could develop spontaneous diastollic depolarization. In this case phase 4 depolarization was followed by a smooth transition into the upstroke of the action potential, strongly suggesting spontaneous action potential generation. Actually this intracellular recording during atrial fibrillation is indistinguishable from a recording during normal sinus rhythm. The cycle length of this example of concealed automaticity in the sinus node during atrial fibrillation was $460 \mathrm{~ms}$, compared to $455 \mathrm{~ms}$ during normal sinus rhythm.

\section{Electrotonic modulation of the pacemaker}

During atrial fibrillation the intracellular recordings from pacemaker fibers frequently showed electrotonic potentials. These potentials resulted from sino-atrial entrance block or dissociated activation between neighbouring groups of sinus node fibers and often disturbed the process of spontaneous diastolic depolarization. In figure 7.6 several examples are given. Recording $A$ shows the occurrence
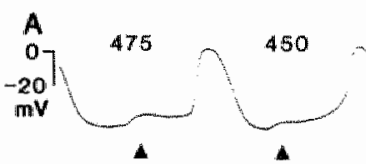

B

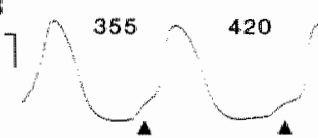

C

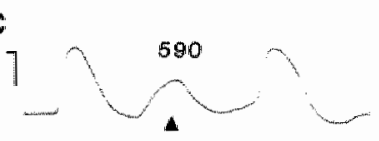

D
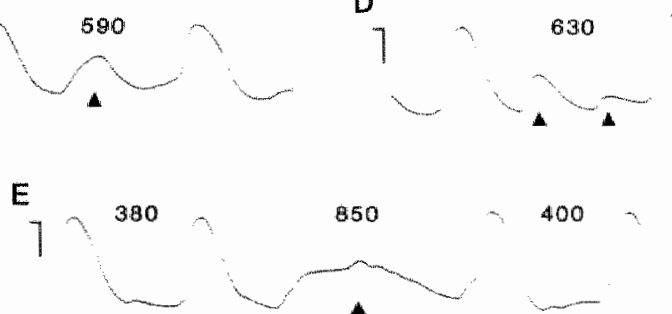

850

400

Figure 7.6:

Electrotonic potentlals (triangles) disturbing diastolic depolarization in pacemaker fibers during atrial fibrillatlon. Tracing A shows the occurrence of single mid-dlastollc potentials. The second activation cycle ends with an evident spontaneous discharge. In tracing B an electrotonic potential was Immedlately followed by the generation of an action potential. Tracing $\mathrm{C}$ depicts a submaxlmal actlon potentlal during a relatively long activation cycle. Tracing $D$ illustrates the occurrence of two successive electrotonic potentials during diastole. Recording E demonstrates multiple superimpased electrotonic potentials leading to prolonged electrotonic depolarization of the fiber. In this example the membrane was kept depolarized during more than $500 \mathrm{~ms}$. This period of inexcitability of the cell resulted in a long cycle length of $850 \mathrm{~ms}$. 
of single mid-diastolic electrotonic potentials, the second one being followed by a spontaneous discharge $450 \mathrm{~ms}$ after the preceding action potential. Tracing $B$ illustrates two subthreshold potentials which were immediately followed by an action potential. The activation cycles were 355 and $420 \mathrm{~ms}$ respectively. Recording $\mathrm{C}$ shows a submaximal action potential occurring between two action potentials of normal configuration, the activation cycle being as long as $590 \mathrm{~ms}$. Recording D depicts a fibrillation cycle which was still longer and demonstrated two successive diastolic potentials. The first response represents a submaximal action potential caused by nearby conduction block. Tracing E shows that multiple electrotonic potentials can keep the cell membrane depolarized during a long period of time. In this example, successive electrotonic depolarizations reduced the membrane potential during more than $500 \mathrm{~ms}$, and thus prevented the generation of an action potential (cycle length $850 \mathrm{~ms}$ ).

During atrial fibrillation the configuration of action potentials in the center of the sinus node changed constantly. In figure 7.7 this beat-to-beat variation is illustrated. Nine successive activation cycles of a pacemaker fiber at $1.5 \mathrm{~mm}$ from the crista terminalis are superimposed. The interval between the successive action potentials varied between 330 and $452 \mathrm{~ms}$ (mean $388 \pm 38 \mathrm{~ms}$ ). The amplitude, rate of rise, duration, maximal diastolic potential and the rate of diastolic depolarization of the action potentials varied considerably.
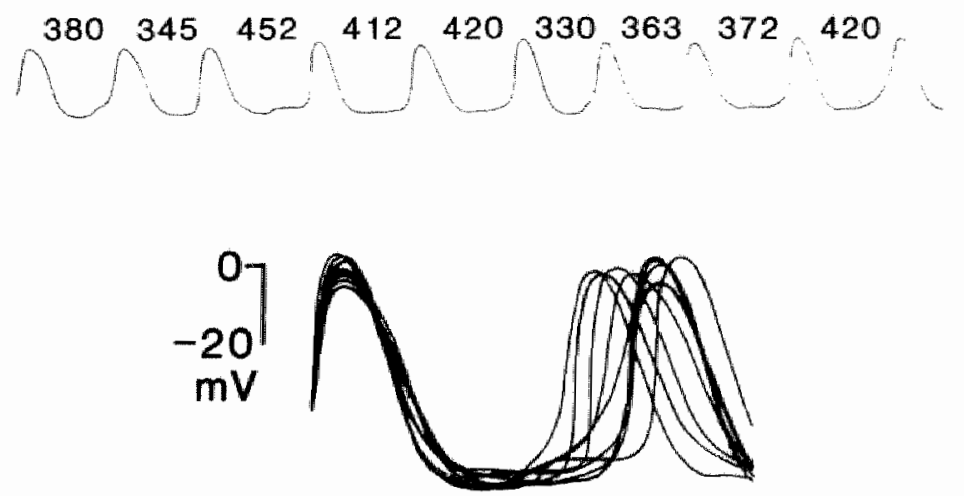

\section{Figure 7.7:}

Beat-to-beat wariation in action potential morphology and cycle length of a pacemaker fiber during atrial fibrillatton. The 9 consecutive cycles th the top tracing are superimposed at the bottom to visualize the variation in actlon potential parameters. The cycle length between the consecutive action potentials is indicated ( $\mathrm{ms}$ ) in the upper tracing. 


\section{Discussion}

\section{Sino-atrial entrance block}

Intracellular exploration of the sinus node during atrial fibrillation revealed a marked heterogeneity in local fibrillation intervals. The average fibrillation interval increased progressively from the atrium to the center of the sinus node. In the border zone the response rate was half the atrial fibrillation rate, which is in proportion to the physiological lengthening of the refractory period in the sinus node. The local intracellular recordings showed well developed action potentials, as well as responses of short duration and reduced amplitude. These brief potentials are typically recorded at the site of local conduction block as demonstrated by Sasyniuk and Mendez [230]. At the transition between border zone and center of the sinus node the local fibrillation rate suddenly decreased even further to less than $20 \%$ of the atrial fibrillation rate. On the average, the activation interval in the pacemaker region was only slightly shorter than during normal sinus rhythm. Evidently, a high degree of entrance block protected the center of the sinus node against penetration of the majority of the fibrillatory impulses. The mechanisms of this protection can not be solely attributed to the longer intrinsic refractory period of sinus node fibers. The marked reduction in fibrillation rate was much larger than could be expected on basis of the local minimal activation cycles during regular pacing. Concealed conduction of fibrillatory wavelets offers a likely explanation for this phenomenon. In several recent studies it was demonstrated that partial penetration of early premature beats into the sinus node or AV node prolongs the refractory period within these structures $[77,148-150,186,215,252]$. In the present experiments concealed conduction of fibrillatory wavelets increased the local refractory period in the border zone. This was itlustrated by action potentials which showed a prolonged duration due to electrotonic potentials from local conduction block. During atrial fibrillation the extent of concealed conduction is influenced by the irregularity in atrial activation and by the interference of wavelets invading the sinus node at different sites [173,174].

The present results are in agreement with the findings of Nelson and Smith in 1960 [192]. In the American bullfrog these authors found that the activity in the sinus venosus was slow and regular during cholinergically induced atrial fibrillation. They related this phenomenon to the sino-atrial junction and speculated that transgression of the fibrillatory impuises through this region was prevented:

\footnotetext{
"...ft, Indeed, there is functioning junctional tissue between the sinus venosus and the atrium, it is probable that the $S$-A locus offers some impedance to the retrograde movement af aberramt impulses from the atrium to the sinus structure. Under these conditions, impulses provoked by fibrillation of the atrial muscle are blocked at the sino-atrial junction, and the sinus venosus will continue to beat quite independenty and at its inherent rate."
} 
Sano et al. [226] found in tissue strips from the isolated rabbit sinus node that spontaneous sinus impulses could trigger short runs of atrial fibrillation. During these runs a high degree of sino-atrial block was observed. Recently, Gomes et all. [66] described the phenomenon of entrance block around the sinus node during atrial fibrillation in man. In a single patient with apparent cardiovascular disease intraatrial recordings demonstrated the existence of sinus rhythm during atrial flutter and fibrillation. In a small area ( $5 \mathrm{~mm}$ diameter) located at the region of the sinus node the activation rhythm was similar to sinus rhythm. This area was protected from the flutter and fibrillation waves by complete entrance block [86].

\section{Concealed automaticity}

In the present experiments the protective entrance block around the central portion of the sinus node drastically reduced the fibrillation rate of pacemaker fibers, the average fibrillation interval being only slightly shorter than the normal sinus interval. Frequently the pacemaker fibers developed diastolic depolarization, sometimes resulting in spontaneous action potential generation. The course of diastolic depolarization was often disturbed by electrotonic potentials of variable amplitude and duration. The occurrence of these potentials can be adequately explained by conduction block of fibrillatory wavelets within the sinus node $[30,249]$. Fibers distal to the site of block are depolarized due to passive flow of current across the area of block. Because of local sino-atrial entrance block, conduction in the center of the sinus node might become dissociated. Different portions of the pacemaker area are then activated out of phase and might influence each other electrotonically. Spontaneous activity in these dissociated areas may result in multiple sites of pacemaking. Although the separate pacemakers might discharge only occasionally, all together they may represent a continuous source of concealed automaticity during atrial fibrillation.

Electrotonic potentials modulate the intrinsic rate of automaticity of cells in the center of the sinus node. This was recently demonstrated by Jalife and coworkers who showed that subthreshold depolarizing impulses, delivered during different moments of diastolic depolarization, may either delay or accelerate the subsequent spontaneous discharge [108-110]. The present intracellular recordings showed that the substrate of pacemaker modulation, i.e. pacemaker activity and subthreshold electrotonic potentials, are both present during atrial fibrillation. 


\section{Sinus node reentry during atrial fibrillation}

During atrial fibrillation incidentally new wavelets emerged from the sinus node (chapter VI). The intracellular recordings from the sinus node during fibrillation offer two possible mechanisms for this phenomenon. First, the new fibrillation waves might represent the exit to the atrium of spontaneous impulses arising from the protected pacemaker fibers in the sinus node. Second, these wavelets might be based on sinus node reentry as a result of dissociated intranodal conduction [5].

Intracellular recordings from the center of the sinus node during atrial fibrillation sometimes suggested spontaneous generation of an action potential. However, this mechanism is rather unlikely to be responsible for the manifestation of sinus impulses during fibrillation since conduction of these impulses towards the atrium is bound to be blocked in the border of the sinus node which is already activated at its highest possible rate. In fact, the border of the sinus node not only protects the center of the node from incoming fibrillation waves, but will likewise prevent spontaneous sinus impulses to exit towards the atrium.

Sino-atrial reciprocation is a more likely explanation for the wavelets emerging from the sinus node during atrial fibrillation. If a fibrillatory wave invades the sinus node, it may propagate through the border zone until the atrium has recovered its excitability again. The results described in chapter III demonstrate that impulse conduction through the border zone is very slow and that during rapid pacing intranodal conduction time may be longer than the refractory period of the atrium. As a result, a fibrillatory impulse after having entered the sinus node at one site and propagating slowly through the border zone, further on may find an exit to the atrium at the moment this is excitable again. The occurrence of partial sino-atrial entrance block in the border zone facilitates reciprocation of atrial impulses [95]. 


\section{Chapter VIII}

RESUMPTION OF SINUS

RHYTHM AFTER

TERMINATION OF ATRIAL

FIBRILLATION 


\section{Introduction}

As demonstrated in chapter $\mathrm{VII}$, the sinus node is still capable of spontaneous impulse generation during atrial fibrillation as a result of the presence of a high degree of sino-atrial entrance block.

In the isolated rabbit heart atrial fibrillation induced by rapid pacing terminated abruptly within 1 to 7 seconds. Termination of fibrillation was followed by the immediate resumption of spontaneous sinus node activity. In a number of experiments we studied the characteristics of recovery of sinus rhythm after cessation of fibrillation. The results of these experiments are described in the present chapter.

\section{Methods}

In 4 experiments short episodes af atrial fibrillation were induced by rapid pacing. The preparation as well as the recording and pacing techniques are described in detail in the 'Methods' section in chapter VI. A single extracellular electrogram from the crista terminalis was selected to analyse the first escape interval and the successive sinus node cycles. During the experiment this electrogram was continuously written on paper (speed $10 \mathrm{~cm} / \mathrm{s}$ ) by means of a conventional penrecorder (Schwarzer RE 412). After the experiment this recording was used to determine the duration of the successive episodes of fibrillation after rapid pacing. If the duration of fibrillation exceeded 1 second, the length of the post-fibrillatory escape cycle (PFEC) and the subsequent spontaneous cycles (SC) were measured by hand. The error of measurement was less than $5 \mathrm{~ms}$. The atrial patterns of excitation of the last few fibrillatory waves and the first escape beats were reconstructed to localize the origin of the escape beats. For a detailed description of the mapping system and the reconstruction of activation maps see the 'Methods' section in chapter VI.

\section{Results}

\section{Sinus node recovery after fibrillation.}

In the present experiments the induced episodes of atriall fibrillation ended abruptly and were followed by immediate resumption of spontaneous activity. The interval between the last fibrillatory activation and the first spontaneous escape beat (PFEC) however varied markedly. It could either be longer, simillar, or markedly 
shorter than the normal sinus node cycle. The interval between the first and second escape beat was mostly prolonged in comparison to normal sinus rhythm. This prolongation gradualiy diminished within the next 20 spontaneous cycles. The atrial activation maps of the first, second and third spontaneous beats after fibrillation showed that these impulses always originated from the region of the sinus node.

In figure 8.1 three examples of the recovery of sinus rhythm are given. Each tracing shows the terminal phase of an episode of atrial fibrillation and the subsecuent resumption of sinus node activity. The last fibrillatory complex in each electrogram is indicated by a black dot. The schemes underneath the signals show the sites of earliest atrial activity during the first three spontaneous beats (black asterisk). For comparison the point of sinus node exit during control sinus rhythm is indicated by an open asterisk. Tracing $A$ shows an example of a relatively long post-fibrillatory escape cycle of $425 \mathrm{~ms}$. The interval between the first and second spontaneous beat was shortened to $370 \mathrm{~ms}$ whereas within the next 5 cycles the interval gradually returned to the basic sinus interval of $330 \mathrm{~ms}$. In this example, the site of origin of the first escape beat was slightly shifted into the direction of the inferior caval vein. During the next spontaneous activations it gradually returned to the position as found during normal sinus rhythm. In panel $B$ the first spontaneous sinus beat occurred at 3"0 ms after cessation of fibrillation. In this case the post-fibrillatory escape interval was slightly shorter than the basic sinus interval of $325 \mathrm{~ms}$. Almost no overdrive suppression was found. Only the interval between the first and second escape beat was slightly prolonged ( $340 \mathrm{~ms})$ and after the second escape beat normal sinus rate was restored again. The morphology of the first sinus escape beat clearly differed from that of the next activations. This was caused by a pacemaker shift to the lower portion of the sinus node. The third example (C) showed an extremely short post-fibrillatory escape cycle of only $150 \mathrm{~ms}$. Its site of exit was located at the superior portion of the crista terminalis, next to the orifice of the superior caval vein. The subsequent activation cycles were slightly prolonged, and gradually returned to the control value of $330 \mathrm{~ms}$. A pacemaker shift was only present during the first escape beat. During the recovery phase the site of exit was the same as during normal sinus rhythm.

\section{Variation in post-fibrillatory escape cycle}

In figure 8.2 the pattern of sinus recovery after termination of fibrillation $(n=231)$ is plotted for the four different experiments. The relative differences with the control sinus interval are plotted for the first six escape cycles. In each experiment the length of the first post-fibrillatory escape cycle (PFEC) varied between a prolongation of 


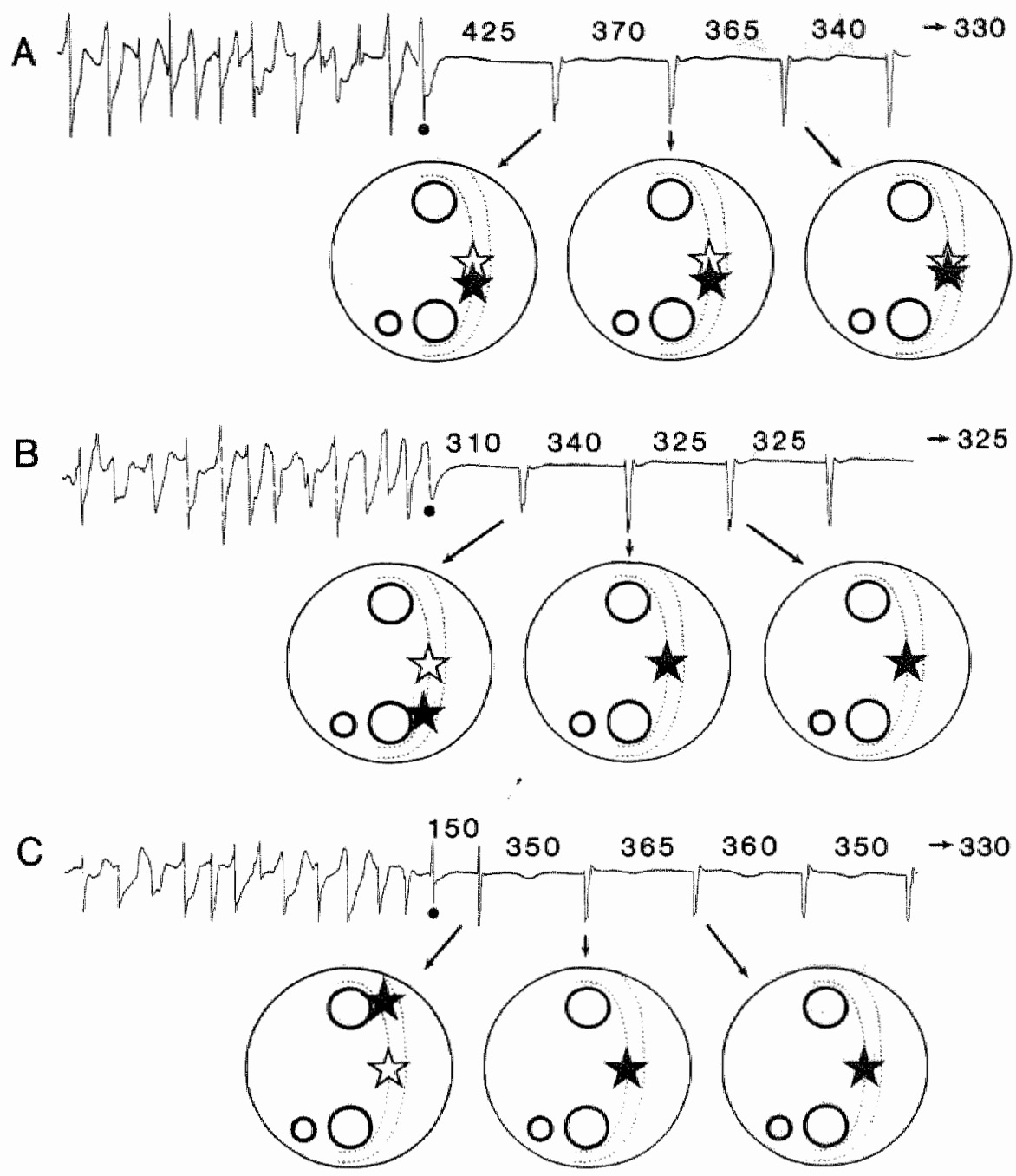

Figure 8.1:

Three episodes of spontaneous termination of atrlal flbrillatlon. The electrogram was recorded from the crista terminalis. The last fibrillatory activation in each panel is indicated by a black dot. The post-fibrillatory escape Interval varied markedly and could be elther longer (A), sllghtly shorter $(B)$, or markedly shorter (C) than the normal sinus Interval whlch Is given at the right of each panel. The schemes show the site of sinus exit (closed asterisk) of the first three escape beats in each panel. The slte of exlt during normal sinus rhythm Is Indicated by open asterisks. 

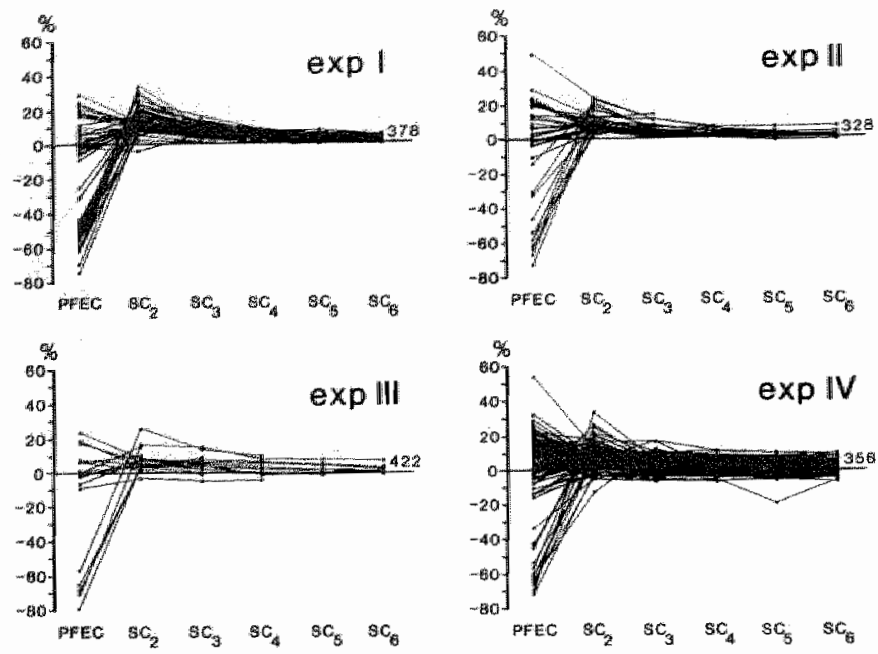

\section{Figure 8.2:}

Sinus node recowery after all episodes of fibrillation in four experiments. The individual curves show the percentual difference of the post-fibrillatory escape cycle (PFEC) and the flve successive sinus cycles $\left(\mathrm{SC}_{2-6}\right)$ with the basic sinus interval. Normal sinus Interval, measured at the $20^{\text {th }}$ spontaneous cycle after each eplsode of fibrillation, is given at the right of the curves. In each experiment a clustering of both long and extremely short past-fibrillatory escape cycles was observed.

about $30 \%$ and a shortening of about $70 \%$. No correlation was found between the duration of the preceding episode of fibrillation and the length of the PFEC. The variation in post-fibrillatory escape cycles was not normally distributed. Instead, a bimodal distribution of relatively long and extremely short cycles was found, whereas post-fibrillatory escape cycles of 10 to $40 \%$ shortening were rare. This abnormai distribution is clearly demonstrated by the histogram of all post-fibrillatory escape cycles (figure 8.3) in which the existence of two subpopulations is obvious. Statistically ( $K$-means Cluster analysis) the left subpopulation in the histogram represents $25 \%$ of the total number of observations and forms a cluster of escape beats which are $-78 \%$ to $-26 \%$ (mean $-55 \pm 20 \%$ ) shorter than control sinus cycle length (mean $365 \pm 30 \mathrm{~ms}$ ). The right part of the histogram shows a subpopulation where the escape interval was $-16 \%$ shorter to $54 \%$ (mean $9 \pm 12 \%$ ) longer than control sinus intervals. This bimodal distribution strongly suggests two different mechanisms underlying the first escape beat after cessation of atrial fibrillation. The average intervals of the two subpopulations and subsequent five sinus cycles $\left(\mathrm{SC}_{2}\right.$ to $\left.\mathrm{SC}_{6}\right)$ in each experiment are summarized in table 8.1. 


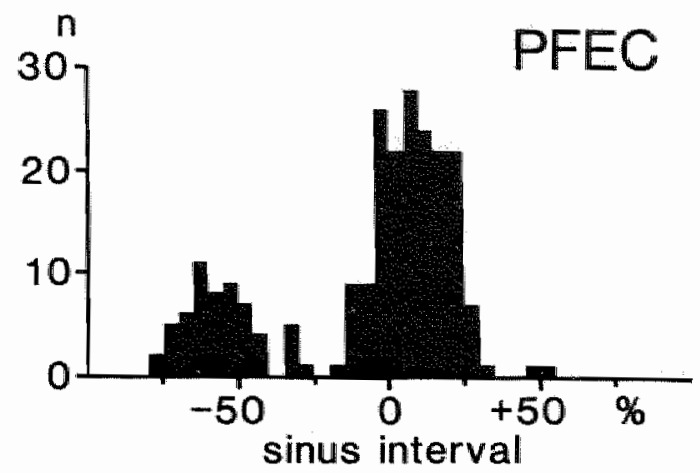

Figure 8.3:

Bimodal histogram of the PFEC after fibrillation. Two distinct populations of post-fibrillatory escape cycles were observed. The left cluster in the histogram represents "early" escape beats, whereas the right cluster represents 'late' escape beats.

\section{Sinus node overdrive suppression during fibrillation}

During atrial fibrillation only a minor portion (20\%) of the wavelets activate the pacemaker fibers in the center of the sinus node (chapter VII) because of a high degree of sino-atrial entrance block. When fibrillation terminates, the last wavelet which penetrates the pacemaker area is not necessarily the last fibrillatory activation in the atrium. Therefore, the post-fibrillatory escape cycle does not represent the actual sinus node recovery time which is normally used to quantify the degree of pacemaker overdrive suppression. We therefore used the second sinus cycle $\left(\mathrm{SC}_{2}\right)$ as a measure to quantify the degree of pacemaker overdrive suppression during fibrillation.

The graph in figure 8.4 shows the average percentual difference of the first 5 sinus cycles $\left(\mathrm{SC}_{2-6}\right)$ compared to normal sinus interval for all experiments. After spontaneous termination of fibrillation the interval of the second sinus cycle $\left(\mathrm{SC}_{2}\right)$ was prolonged but gradually returned to normal sinus interval within the next few

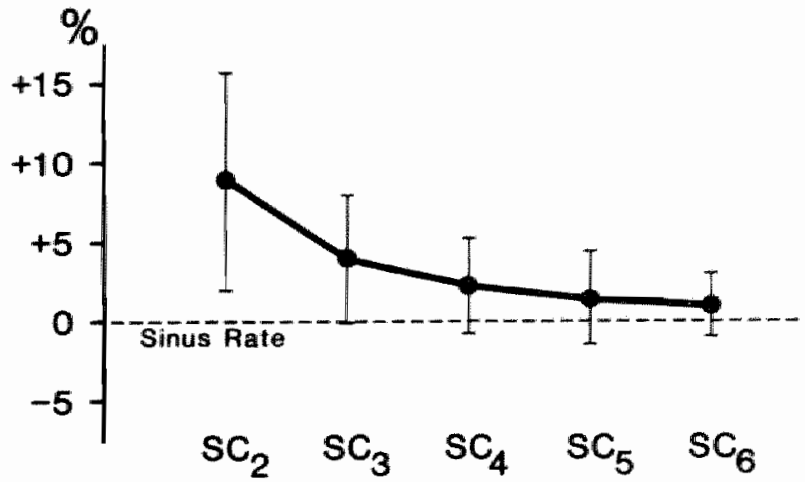

Figure 8.4:

Overdrlwe suppression of the sinus pacemaker during atrial flbrlltation. The average prolongation of the second to shth sinus cycle after spontaneous termination of fibrllation Is represented as mean \pm SD. Absolute walues are giwen in table 8.1 .

\section{$\begin{array}{lllll}\mathrm{SC}_{2} & \mathrm{sC}_{3} & \mathrm{SC}_{4} & \mathrm{sC}_{5} & \mathrm{SC}_{6}\end{array}$}


Table 8.1.

Sirus Restoration After Spontaneous Termination Of Atrial Fibrillation.

\begin{tabular}{lccccccccc} 
EXP & AF-dur & \multicolumn{2}{c}{ PFEC } & $\mathbf{S C}_{2}$ & $\mathbf{S C}_{3}$ & $\mathbf{S C}_{4}$ & $\mathbf{S C}_{5}$ & $\mathbf{S C}_{6}$ & $\mathbf{S C L}$ \\
$\mathbf{I}$ & & 'E' & 'L' & & & & & & \\
mean & 2074 & 178 & 409 & 431 & 406 & 395 & 389 & 386 & 378 \\
SD & 1374 & 39 & 42 & 26 & 15 & 13 & 12 & 12 & \\
$\mathbf{n}$ & 55 & 28 & 27 & 55 & 54 & 54 & 54 & 53 &
\end{tabular}

$\begin{array}{lccccccccc}\text { II } & & & & & & & & & \\ \text { mean } & 2147 & 149 & 363 & 359 & 343 & 337 & 334 & 332 & 328 \\ \text { SD } & 1269 & 43 & 45 & 19 & 13 & 8 & 8 & 9 & \\ \text { n } & 37 & 10 & 27 & 36 & 28 & 17 & 15 & 12 & \end{array}$

$\begin{array}{lccccccccc}\text { III } & & & & & & & & & \\ \text { mean } & 1447 & 135 & 461 & 452 & 442 & 434 & 432 & 432 & 422 \\ \text { SD } & 485 & 27 & 50 & 28 & 23 & 21 & 16 & 13 & \\ \text { n } & 29 & 6 & 23 & 18 & 14 & 10 & 8 & 7 & \end{array}$

$\begin{array}{lccccccccc}\text { IV } & & & & & & & & & \\ \text { mean } & 2255 & 152 & 388 & 379 & 363 & 360 & 358 & 357 & 356 \\ \text { SD } & 1213 & 44 & 40 & 31 & 26 & 26 & 25 & 24 & \\ \text { n } & 110 & 15 & 95 & 110 & 110 & 110 & 110 & 109 & \end{array}$

Mean and SD given In ms. AF-dur: cluration of the fibrillatlon eplsode; PFEC: Post-fibrillatory escape cycle:" $E$ ': early escape beats; ' $L$ ': late escape beats; $S_{2-6}$ : Sinus cycle 2 to 6 .

beats. This pattern of sinus node recovery indicates the occurrence of pacemaker overdrive suppression during atrial fibrillation. On the average, the $\mathrm{SC}_{2}$ was lengthened by $14 \%$ and by $9 \%$ in experiments I and II, and by $7 \%$ in experiments III and IV. The average prolongation of the $\mathrm{SC}_{2}$ in all experiments was $9.0 \pm 7 \%$ (mean \pm SD). No statistical correlation (Pearson) was found between the second spontaneous cycle $\left(\mathrm{SC}_{2}\right)$ and the duration of preceding fibrillation.

In one experiment (exp IV in figure 8.2) sinus recovery frequently showed sinus overdrive acceleration after termination of fibrillation, the second spontaneous sinus 
cycle length being slighty shorter than basic sinus interval. At the $20^{\text {th }}$ spontaneous beat basic sinus rate was always restored.

\section{Discussion}

\section{Sinus overdrive suppression}

After a period of regular atrial pacing with a rate higher than sinus rate, the sinus node recovery time is used as a measure of pacemaker overdrive suppression $[120,147,251,272]$. This parameter increases at higher pacing rates until the maximal sinus recovery time is reached. Further increase of the pacing rate causes a decrease in the duration of the post-pacing pause $[140,168,251]$. This results from the development of sino-atrial entrance block which decreases the effective rate of overdrive of the pacemaker in the center of the sinus node. Under these conditions the length of the sinus recovery cycle depends strongly on the fact whether the last stimulated atrial impulse activates the sinus pacemaker or is blocked in the sino-atrial border zone [251]. A similar situation exists during atrial fibrillation. After termination of fibrillation sinus recovery was characterized by a slight amount of overdrive suppression. In the present experiments the sinus escape beats sometimes showed a post-fibrillatory interval which was slightly shorter than the cycle length during sinus rhythm. This phenomenon was also described by Kodama and Toyama after periods of rapid atrial pacing [140]. In the present experiments the post-fibrillatory escape beats and a varying number of subsequent beats showed a different site of sinus exit than during sinus rhythm. This strongly suggests a shift of the pacemaker within the sinus node after termination of fibrillation. This is in agreement with the observed pacemaker shift after regular atrial pacing, single premature atrial depolarizations, and sinus node reentry $[5,30,251]$.

Overdrive suppression of the sinus pacemaker after short periods of atrial fibrillation was illustrated by an average prolongation of the second escape interval $\left(\mathrm{SC}_{2}\right)$ of $9 \%$. We could not compare these results with other studies. No literature is available providing data on the degree of post-fibrillatory overdrive suppression estimated by measurement of the first spontaneous cycle length during sinus node recovery.

In a few cases a slight and transient pacemaker acceleration was found after the first sinus escape beat. This phenomenon was also described by Lange [147] and Jordan et al. [120] after atrial pacing in dogs. Pacing-induced catecholamine release was suggested to be the underlying mechanism [147]. 


\section{Bimodal distribution of the first post-fibrillatory escape beat}

In the present experiments atrial fibrillation lasted only several seconds, terminated abruptly and was followed by immediate resumption of sinus rhythm. This is in agreement with earlier reports on termination of atrial fibrillation $[18,147,162,281]$. Mapping of atrial activation revealed that the post-fibrillatory impulses always originated from the sinus node region. The site of sinus exit of the first escape beats however was different from that during stable sinus rhythm. Sometimes this shift in sinus exit was confined to the first escape beat, in other cases it persisted during a variable number of sinus beats. The interval between the last fibrillatory activation in the crista terminalis and the first escape beat (PFEC) varied widely. Sometimes it was very short and hardly exceeded the foregoing fibrillation intervals, in other cases it showed a marked prolongation in comparison to the pre-fibrillatory sinus intervals. The histogram of the post-fibrillatory escape cycle showed two distinct populations of intervals with peak values at $-55 \%$ ('early' escape beats) and $+9 \%$ ('late' escape beats) of the cycle length during normal sinus rhythm. This bimodal distribution strongly suggests two different mechanisms of post-fibrillatory sinus escape.

\section{'Late' sinus escape beats}

During atrial fibrillation the high degree of sino-atrial entrance block reduces the activation rate in the pacemaker fibers far below the maximal rate during regular pacing, the average rate in the center of the sinus node being only slightly faster than during normal sinus rhythm (chapter VII). Intracellular recordings further demonstrated that the activation of pacemaker fibers during atrial fibrillation was quite irregular showing a marked variation in beat-to-beat cycle length. As a result, the degree of overdrive suppression can be expected to vary in time. In addition, also the moment of the last discharge of the pacemaker prior to termination of fibrillation will play an important role. If the overdrive suppression at the moment of termination is relatively high and if the pacemaker was just activated by one of the last fibrillation waves, a relativelly long post-fibrillatory escape cycle can be expected (panel A, figure 8.1). If, however, tibrillation terminates while the last fibrillating waves have been blocked in the sino-atrial border, the generation of the first spontaneous escape impulse has already been started prior to cessation of fibrillation. This will result in an escape interval shorter than the normal sinus interval. This type of sinus escape however is limited by the refractory period of the sino-atrial border zone. If the pacemaker discharges before the excitability of the border zone is restored, exit block of the sinus impulse will occur. 


\section{'Early' sinus escape beats}

The fibrillation rate in the border zone of the sinus node appeared to be almost as high as the maximal regular pacing rate in this area. As a consequence, transmission of spontaneous impulses from the center of the sinus node towards the atrium is practically impossible during fibrillation. This implicates that the early postfibrillatory escape beats are unlikely to be based on pacemaking in the center of the sinus node. Spontaneous impulse formation in the border zone is improbable since we recorded no spontaneous diastolic depolarization in these fibers during atrial fibrillation (chapter VII).

Triggered activity based on delayed afterdepolarizations represents another mechanism explaining the occurrence of early post-fibrillatory escape beats. Several investigators observed oscillating potentials after the repolarization phase in cardiac fibers, particularly after digitalis administration $[55,89,218]$. If these potentials reach threshold, an early action potential is generated which manifestates as a premature activation [89]. Although in the present experiments no digitalis was used and no evidence was obtained for abnormal automaticity or oscillating afterpotentials in sinus node fibers, the occurrence of triggered activity could not be excluded.

A more likely explanation is that early escape beats are caused by sino-atrial reciprocation (sinus echo beats). The high incidence of intranodal conduction block certainly favours the occurrence of sinus node echo beats. If the last fibrillatory wave encounters entrance block at one site of the sinus node, it may invade the sinus node from another site and propagate through the border zone to activate the area of entrance block in retrograde direction. Since conduction velocity in the border zone is relatively low (chapter III), by that time atrial excitability has recovered and the impulse will exit the sinus node again. In that case the post--fibrillatory escape cycle represents the time of sino-atrial reciprocation of the last fibrillatory impulse. In three experimental studies using the isolated rabbit sinus node, echo beats showed atrial return cycles which varied between 125 and $225 \mathrm{~ms}[5,95,256]$. This corresponds to the range of early post-fibrillatory escape cycles as found in the present experiments.

In the present experiments early sinus escape beats were found in $25 \%$ of all episodes of fibrillation. This indicates that sinus node reentry during atrial fibrillation might be a common phenomenon. During sustained fibrillation sinus echoes can only reenter the atrium within a short time period between the successive fibrillatory activations (chapter VI). Indications for sinus echoes during fibrillation were found in one out of ten atrial activations. This means that only $40 \%$ of the reciprocating wavelets reenter the atrium during fibrillation. The other impulses do not find an exit to the atrium and block in the sino-atrial border zone. 
Extracellular mapping of the atrium and intracellular recordings from the sinus node have demonstrated that during atrial fibrillation the sinus node acts as a source of impulses increasing the average number of impulses. This mechanism facilitates the perpetuation of the fibrillatory process and might easily restart the arrhythmia at a moment of simultaneous cancelation of all wavelets. Sinus echo beats are the likely mechanism underlying sinus impulses during fibrillation. When fibrillation terminates, concealed generation of spontaneous impulses and currently reciprocating wavelets manifestate as initial sinus escape beats which come earlier than expected on basis of the previous sinus rate. 


\section{Chapter IX}

GENERAL DISCUSSION 


\section{Synopsis}

The present thesis describes the results of experimental studies in which the sino-atrial transitional zone forms the region of interest. Different aspects of sinus automaticity and conduction of the impulse in the sinus node were investigated in the isolated rabbit heart. Furthermore, the behaviour of the sinus node during atrial fibrillation was studied in an attempt to elucidate a possible role of this structure in the perpetuation of atrial fibrillation.

In the mammalian heart the sinus node is electrically coupled to a relatively large atrial tissue mass. Since electrically coupled fibers might demonstrate mutual electrotonic modulation we studied the passive electrical influences of the crista terminalis on spontaneous impulse formation in the isolated right atrium of the rabbit heart. The site of impulse origin and the spread of excitation within the sinus node was reconstructed and compared to the location of pacemaking and activation pattern after surgical disconnection of the sinus node and the crista terminalis. Before sino-atrial separation the site of impulse origin was located in the central portion of the sinus node and coincided with the spot where fibers exhibited the steepest slope of spontaneous diastolic depolarization. Disconnection of the sinus nade from the atrial myocardium induced a permanent increase in sinus rate by about $20 \%$. Reconstruction of the activation pattern in the sinus node revealed a simultaneous shift of the pacemaker from the center of the sinus node towards a new location in the sino-atrial border zone. This pacemaker shift was accompanied by a marked steepening of the spontaneous diastolic depolarization in the entire border zone. Since this increase in diastolic depolarization rate did not occur in the nodal center, the border zone fibers became the dominant pacemaker fibers in the sinus node. Among other changes in action potential configuration, these findings strongly suggested that the atrial myocardium normally reduces the rate of automaticity in border zone fibers by an electrotonic depression of the slope of spontaneous diastolic depolarization. We concluded that the intrinsic pacemaker properties of fibers in the border zone of the sinus node are equivalent or even better than those of the dominant pacemaker fibers in the center of the sinus node. These findings implicate that changes in electrical coupling between atrial and sinus node fibers might cause pacemaker shifts within the sinus node and lead to sinus arrhythmias.

Proper sinus function basically depends on adequate impulse formation, followed by undisturbed conduction of the impulse towards the atrial myocardium. In the isolated rabbit sinus node preparation this sino-atrial conduction can be studied directly manner by intracellular mapping of the nodal activation pattern, or indirectly by programmed atrial stimulation. However, an important prerequisite for a reliable application of both techniques is a fixed pacemaker position within the sinus 
node and a stable sinus rhythm. In comparative studies on the effects of autonomic transmitters, drugs, altered electrolyte concentrations or other environmental changes this prerequisite is rarely met. In the present thesis an alternative technique was demonstrated. This model allows investigation of sino-atrial conduction properties, independently of the location and rate of sinus automaticity, and without the necessary use of microelectrode techniques. The atrial portion of the isolated rabbit sinus node preparation was divided in two halves by an incision perpendicular to the crista terminalis and ending in the border zone or center of the sinus node. Thus, conduction of a stimulated atrial impulse through the sinus node - which connected both atrial halves - could be studied. It was assumed that the impulse propagated always along the shortest pathway through the sinus node, i.e. just around the tip of the incision. This assumption was validated by reconstruction of the conduction route through the sinus node.

It appeared that the conduction velocity of an impulse through the border zone of the sinus node is much lower than in the atrium and is characterized by a clear rate-dependent decrease at higher rhythms. During maximal regular pacing the conduction velocity through the border zone was almost halved compared to the conduction velocity during slow pacing with an interval only slightly shorter than the spontaneous sinus cycle length. The refractory period in the sino-atrial border zone during slow pacing. The maximal activation rate $\left(\boldsymbol{F}_{\max }\right)$ in the border zone during regular pacing was about half the maximal rate in the atrium. In the center of the sinus node impulse conduction was slower than in the border zone and showed the most prominent rate-dependency. The refractory period in the center of the sinus node was considerably longer than in the border zone. In the present experiments it was demonstrated that the transnodal conduction time during rapid pacing and perticularly of an early premature atrial impulse may exceed the refractory period of the atrium. This implies that a premature atrial impulse can travel through the sinus node until the atrium has restored excitability. In this moment the impulse may exit the sinus node and reexcite the atrium. This is in agreement to the findings of $H a n$ et al [95] and Allessie and Bonke [5] who demonstrated sinus reciprocation in the isolated rabbit sinus node. However, the occurrence of sustained sinus reciprocation or sinus node reentry is unlikely. For sustained sinus reciprocation to maintain a relatively long pathway through the fast conducting atrium is required before the sinus node has restored excitability and can be reinvaded again. Such a pathway does not fit into the atrium of the rabbit. In case of sustained reentrant excitation within the sinus node itself, the center of the node would be incorporated in the reentrant pathway. Since the maximal pacing rate in this region of the sinus node is very low, the impuise would have to propagate during a long time period through the sino-atrial border zone before the center of the node can be reexcited. How- 
ever, this detour would probably exceed the dimensions of the sino-atrial border zone in the rabbit sinus node.

The applicability of the present method to study sinus node conduction properties was illustrated by examination of the effects of graded hypothermia, moderate hypoxia, and autonomic transmitters. Both hypothermia and moderate hypoxia caused a decrease in conduction velocity and a prolongation of the refractory period in the sinus node. Autonomic transmitters affected impulse conduction through the sino-atrial border zone only to a limited extent. This was in contrast to the effects on conduction through the center of the sinus node. Norepinephrine accelerated impulse conduction and shortened the functional refractory period, whereas acetylcholine reduced the conduction velocity and prolonged refractoriness in the pacemaker region of the sinus node.

The actions of four antiarrhythmic drugs - bepridil, verapamil, diltiazem and disopyramide on conduction properties in the sinus node and atrial myocardium were studied. All agents exhibited a typical profile of actions. Bepridil in a relatively narrow concentration range $\left(1\right.$ to $\left.3 \times 10^{-6} \mathrm{M}\right)$ reduced the conduction velocity and leng. thened the refractory period in the sinus node markedly. However, sinus rate was hardly affected with these drug concentrations. When the concentration was elevated to 4 or $6 \times 10^{-6} \mathrm{M}$, sinus automaticity suddenly ceased whereas atrial conduction properties became markedly depressed. With these higher concentration conduction through the sinus node was completely blocked. These results strongly suggest that bepridil possesses both slow and fast channel blocking properties. Verapamil $\left(2.2\right.$ to $\left.8.8 \times 10^{-8} \mathrm{M}\right)$ exhibited similar actions on sino-atrial conduction properties. A marked reduction in conduction velocity and a considerable prolongation in refractoriness were accompanied by only a minimal effect on sinus rate. However, the effective concentrations of verapamil were almost 50 times lower than those of bepridil. Furthermore, verapamil in concentrations up to $17.6 \times 10^{-8} \mathrm{M}$ did not affect atrial conduction properties. Diltiazem $\left(5 \times 10^{-7}\right.$ to $\left.5 \times 10^{-6} \mathrm{M}\right)$ in concentrations similar to bepridil showed an equal depression of sino-atrial conduction properties and sinus automaticity. Diltiazem did not affect conduction and refractoriness in the atrium. Disopyramide was tested in a relatively wide concentration range $\left(1 \times 10^{-6}\right.$ to $\left.5 \times 10^{-5} \mathrm{M}\right)$ and depressed sino-atrial conduction only at high dosages. However, this drug demonstrated a marked effect on atrial conduction properties.

The role of the sinus node in the genesis, perpetuation and termination of atrial fibrillation has been subject of speculations in several earlier studies $[192,226,235,243]$. In the present thesis the results of an extensive study on this issue are described. In the isolated Langendorff perfused rabbit heart we used extracellular mapping techniques in combination with intracellular recordings to obtain direct information 
on the electrical activity in the atrium around the sinus node and in the sinus node itself during electrically induced episodes of atrial fibrillation.

Reconstruction of the atrial activation sequence during fibrillation showed that the sinus node region was continuously approached by multiple activation waves. These wavelets entered the mapping area from various directions and with an average interval of about $80 \mathrm{~ms}$. However, it appeared that the sinus node could also act as a source of wavelets. About once per second a wavefront arose from the edge of the sinus node and participated in the fibrillatory process. These impulses could not be deducted directly from the propagation of previous atrial fibrillation waves. This phenomenon increased the average number of wandering wavelets which might facilitate the perpetuation of atrial fibrillation.

To study the electrical activity during atrial fibrillation in the sinus node itself, intracellular registrations were obtained from different nodal regions. It appeared that the cellular activation rate in the border zone of the sinus node was only half the atrial fibrillation rate. The intracellular registrations showed a high incidence of electrotonic responses which indicated a frequent occurrence of local conduction block in the barder zone. In the center of the sinus node the activation rate was still lower than in the border zone and even hardly exceeded the sinus rate before atrial fibrillation. On the average about one out of five atrial fibrillation waves penetrated the pacemaker region. In the border zone the average response interval was similar to the local interval $F$ max. However, in the center of the sinus node the mean fibrillation interval was much longer than the local interval $F_{\text {max. Apparently, the high degree }}$ of sino-atrial entrance block in the borcler zone protected the pacemaker fibers in the center of the sinus node against the rapid fibrillatory rhythm in the atrium. As a consequence, the pacemaker cells in the center could still develop spontaneous diastolic depolarization although often disturbed by electrotonic potentials from local conduction block in the vicinity of the nodal center. Nevertheless, this spontaneous diastolic depolarization occasionally resulted in spontaneous action potential generation during atrial fibrillation. However, this concealed automaticity could not explain the wavelets originating from the sinus node during atrial fibrillation. The high degree of sino-atrial entrance block not only protected the pacemaker fibers against the high fibrillatory rate, but also prohibited spontaneous impulses to exit from the nodal center.

A more likely explanation for the origination of sinus impulses during atrial fibrillation was given by the occurrence of sinus reciprocation. The high incidence of local sinus entrance block favoured the occurrence of sinus echo beats. This was strengthened by the findings on the recovery of sinus rhythm after spontaneous termination of atrial fibrillation. It was found that in a quarter of cases the first sinus escape beat after the last atrial fibrillatory activation occurred much earlier than ex- 
pected on basis of the pre-fibrillatory sinus interval. Since the early sinus escape beats represented a distinct subpopulation in a bimodal distribution of post-fibrillatory escape intervals, they were strongly suggested to be based on a mechanism different from normal sinus automaticity. Probably these early sinus escape beats were the result of sinus reciprocation of one of the last fibrillatory wavelets. This could occur since conduction of the impulse through the border zone of the sinus node at high activation rates is very slow and may easily exceed the refractory period of the atrium. If the last fibrillation wave propagates through the sinus node it may find an exit to the atrium again as soon as this has recovered excitability. The atrial reentrance will manifest as an early sinus escape beat.

The results of the experiments on the role of the sinus node during atrial fibrillation suggested that the sinus node might facilitate the perpetuation of the fibrillatory process by acting as a source of reciprocating impulses. Furthermore, the early escape beats after termination of fibrillation might easily restart the fibrillatory process. The high degree of sino-atrial entrance block reduces the degree of sinus node overdrive suppression which results in a prompt resumption of sinus rhythm after spontaneous termination of atrial fibrillation. 



\section{List of References}

1. Adam H. Experimentelle Untersuchungen uber den Ausgangspunkt der automatischen Herzrelze belm Warmbluter. Pflügers Arch Ges Physlol 111:607-619, 1906.

2. Allessie MA, Bonke FIM, Schopman FJG. Clrcus movement in rabbit atrial muscle as a mechanism of tachycardia. Circ Res 32:54-62, 1973.

3. Allessie MA, Bonke FIM, Schopman FJG. Circus movement in rabbit atrial muscle as a mech. anism of tachycardia. II. The role of nonuniform recovery of excitability in the occurrence of unidirectional blocks as studied with multiple microelectrodes. Circ Res 39:168-177, 1976.

4. Allessie MA, Bonke FIM. Schopman FJG. Circus movement in the rabbit atrial muscle as a mechanism of tachycardia III. The "leading circle" concept: A new model of circus movement in cardiac tissue without the Involvement of an anatomical obstacle. Circ Res 41:9-18, 1977.

5. Allessie MA, Bonke FIM. Direct demanstration of sinus node reentry in the rabbit heart. Circ Res 44:557-568, 1979.

6. Allessie MA, Lammers W.JEP, Bonke FIM, Hollen J. Experimental evaluation of Moe's multiple wavelet hypothesis of atrlall fibrillation. In: Cardiac electrophysiology and arrhythmias. Eds: Zipes DP, Jalife J. Grune \& Stratton, Orlando " pp 265-275, 1985.

7. Allessle MA, Hoeks APG, Schmitz GML, Reneman RS. On-line mapping system for the visuallzation of the electrical activation of the heart. Int J Cardiac Imag 2:59-63, 1986.

8. Allessie MA, Lammers WJEP, Rensma PL, Bonke FIM. Flutter and fibrillation in experimental models: What has been learned that can be applledi to humans? In: Cardlac Arrhythmlas: Where to go from here? Eds: Brugada P, Wellens HJJ. Futura, Mount Klsco, New York, pp 67$82,1987$.

9. Alpert JS, Coumel P, Greeff K, Krtkler DM, Remme WJ, Schönbaum E, Verduyn CW. Bepridl:: a revlew of its pharmacology and clinical,efflcacy as an antl-anginal agent with ant-arrhythmlc properties. Pharmatherapeutica 4:195-222, 1985 .

10. Andrus EC, Carter EP. The refractory period of the normally-beatling dog's auricle; with a note on the occurrence of auricular fibriliation following a single stimulus. J Exp Med 51:357-364, 1930.

11. Anno T. Furuta T, Itho M, Kodama $I$, Toyama J, Yamada K. Effects of beprldill on the electrophysiological propertles of guinea-plig ventricular muscles. Br J Pharmac 81:589-597, 1984,

12. Antzelevitch $\mathrm{C}$, Jallife J, Moe GK. Electrotonlc modulation of pacemaker activity. Further blologlical and mathematlcal observations on the behavlor of modulated parasystole. Circulation $66: 1225-1232,1982$.

13. Azuma $K_{n}$ Shinmura $H$, Shimlzu K. Signtficance of the sino-atrial node on the mechanism of occurrence of atrial fibrillation. Jpn Heart $\sqrt{ } 13: 84-98,1972$.

14. Barker PS, Wilson FN, Johnson FD. The mechanlsm of aurlcular paroxysmal tachycardla. Am Heart J 26:435-445, 1943.

15. Bashour T. Classiffcation of sinus node dysfunction. Am Heart J 110:1251-1256, 1985.

16. Bayliss WM, Stanling EH. On the electromotive phenomena of the mammallan heart. Month int Anat Physilol 9:256-281, 1892.

17. Beaughard $M_{3}$ Ferrler $M_{*}$ Labrid $C_{\text {, Lamar }} \mathrm{C}_{n}$ Leboeuf $\mathrm{d}$, Pirls $P$. Studles on the bradycardia induced by bepridli. Br J Pharmacol 75:293-300, 1982.

18. Biennett MA, Pentecost $B L$. The pattern of onset and spontaneous cessation of atrial fibrillation In man. Circulation 39:981-988, 1970. 
19. Bharatl $S$, Nordenberg $A$, Bauernfiend $A$, Varghese JP, Carvalho $A G$, Rosen $K$, Lev $M$. The anatomic substrate for the sick sinus syndrome in adolescence. Am J Cardiol 46:163-172, 1980.

20. Bigger JT, Relffel JA. Sick Sinus Syndrome. Ann Rev Med 30:91-118, 1979.

21. Eirkhead JS, Vaughan Willams EM. Dual effect of disopyramide on atrial and atrioventricular conduction and refractory periods. Br Heart $J 39: 657-660,1977$.

22. Blair $S M$, Davies $F$. Observations on the conducting system of the heart. $J$ Anat 69:303-324, 1935.

23. Bleeker WK, Mackaay AJC, Masson-Pevet M, Bouman LN, Becker AE. Functionall and morphological organization of the rabbit sinus node. Circ Res 46:11-22, 1980.

24. Bleeker WK, Mackaay AJC, Masson-Pevet M, Op "t Hof T, Jongsma HJ, Bouman LN. Asymmetry of the sino-atrial conduction in the rabbit heart. J Mol Cell Cardiol 14:633-643, 1982 .

25. Boineau JP, Schüssler RB, Mooney CR, Wylds AC, Miller CB, Hudson RD, Borremans JM, Brockus CW. Multicentric origin of the atrial depolarization wave: The pacemaker complex. Relation to dynamics of atrlal conduction, $\mathrm{P}$-wave changes and heart control. Circulation 58 : $1036-1048,1978$.

26. Boineau JP, Schüssler RB, Hackel DB, Miller CB, Brockus CW. Wylds AC. Widespread distributhon and rate differentlatlon of the atrial pacemaker complex. Am J Physiol 239:H406-H415. 1980 .

27. Boineau JP, Schüssler RB, Roeske WR, Autry LJ, Miller CB, Wylds AC. Quantitative relation between sites of atrial Impulse origin and cycle length. Am J Physlol 245:H781-H789, 1983.

28. Bolneau JP, Schüssler RB, Roeske WR, Miller CB, Wylds AC, Hill DA. Autonomic organization of the atrial pacemaker complex. In: Cardiac electrophysiology and arrhythmias. Eds: Zipes $\mathrm{DP}_{n}$, Jallife ل. Grune and Stratton, Orlando, pp 151-158, 1985.

29. Bonke FIM, Bouman LN, van Aijn HE. Change of cardiac rhythm in the rabbit after an atrial premature beat . Circ Res 24:533-544, 1969.

30. Bonke FIM, Bouman LN, Schopman FJG. Effect of an early atriall premature beat on activity of the sinoatrlal node and atrial rhythm in the rabbit. Clrc Res 29:704-715, 1971.

31. Bonke FIM. Electrotonic spread in the sinoatrial node of the rabbit heart. Pfügers Arch $339: 17-$ 23,1973

32. Bonke FIM. Electrophyslology of the sinus node and atrial pacemakers. In: Physlology of atrial pacemakers and conductive tlssues. Ed: Little RC. Futura Publlishing Company, Mount Klsco, New York, pp 171-186, 1980.

39. Bonke FIM, Stelnbeck G, Allessle MA, Mackaay AJIC, Slenter VAJ. The el ectrophysiological effects of cardlec glycosides on the isolated sinus node of the rabbit. In: Normal and abnormal conduction in the heart. Eds: Paes de Carvalho A, Hoffman BF, Lieberman M. Futura PublishIng Company, Mount Kisco, New York, pp 347-361, 1982.

34. Boucher M, Duchene-Marullaz P. Comparative effects of amiodarone, perhexlline and bepridil on the cardlac rhythms of the unanesthetized dog in chronlc heart block. Arch Int Pharmacodyn Ther 233:65-75, 1978.

35. Bouman LN. De werking van de nervus vagus op de prikkelvorming in de sino-auilculalire knoop (The action of the vagal nerves on the pacemaker activity of the sino-auricular node). Thesis, Amsterdam, 1965.

36. Bouman $L N$, Gerlings ED, Blersteker PA, Bonke FIM. Pacemaker shift in the sino-atrial node during vagal stimulation. Pfluggers Arch 302:255-267, 1968. 
37. Bouman LN, Mackaay A.JC, Bleeker WK, Becker AE. Pacemaker shifts in the sinus node: effects of vagal stimulation, temperature and reduction of extracellular calcium. In: The sthus node. Structure, function and clinical relevance. Ed: Bonke FIM. Martinus Nijhof, The Hague, pp 245$257,1978$.

38. Bouman $L N$, Op 't Hof T. Mackaay AJC, Bleeker WK, Jongsma HJ. On the intrinsic cardiac rhythm. In: Cardliac Rate and Rhythm. Eds: Bouman LN, Jongsma HJ. Martinus Nijhoff, The Hague, pp 473-483, 1982.

39. Bouman $L N$, Jongsma $H J d$. Structure and function of the sino-atrial node: a review. Eur Heart $J$ 7:94-104, 1986.

40. Breithardt $G_{a}$ Selpell $L$ Loogen $F$. Sinus node recovery time and calculated sinoatrial conduction time in normal subjects and pattents with sinus node dysfunction. Circulation 56:43-50, 1977.

41. Breithardt $G$, Selpel $L$, Wiebringhaus $E$. Dual effect of verapamill on sinus node function in man. In: The sinus node. Structure, function and cllinical relevance. Ed: Bonke FIM. Martinus Nillhoff, The Hague, op 129-138, 1978.

42. Breithardt $G$, Selpel L. Further evidence for the site of reentry in so-called sinus node reentrant tachycardia In man. Eur J Card 11:105-113, 1980.

43. Breithardt $G$, Selpel $L$. Role of sinus node reentry in the genesis of supraventrlcular arrhythmias. In: Cardiac electrophysiology today. Eds: Masonil A, Albonll P. Academic Press, London, New York, pp 99-122, 1982.

44. Brown HF, Glles W, Noble SJ. Membrane currents underlying activity in frog sinus venosus. J Physiol London 271:783-816, 1977.

45. Bukauskas FF, Veteikis RP, Gutman AM, Mutskus KS. Intracellular coupling in the sinus node of the rabblt heart. Biofizika 22:108-112, 1977.

46. Castellanos $A_{n}$ Aranda J, Molelro $F$, Mallon SM, Befeler B. Effects of the pacing site In sinus node reentrant tachycardla. J Electrocardiol 2:165-169, 1976.

47. Chassaing $C_{1}$ Molns $N$, Lavarenne J, Duchene-Marullaz $P$. Effets compares du bepridll, de la perhexilline de l'amiodarone et de la quinidine sur les periodes refractaires auriculaires et auriculo-ventriculaires du chlen narcose. J Pharmacol (Paris) 8:503-514, 1977.

48. Chiba S, Suzuki $Y$, Hashimoto K. Atrial fibrillation induced by infusion of hypertonlc solutions into the canine sinus node artery in situ. J Parmacol Exp Ther 167:274-281, 1969.

49. Childers RW, Arnsdorf MF, Fuente dela DJ, Gambetta M, Svenson R. SInus nodal echoes. Cilntcall case report and canine studles. Am J Cardlol 31:220-231, 1973.

50. Clapham DE, Shrler A, DeHaan RL. Junctional resistance and action potentlal delay between embryonic heart cell aggregates. J Gen Physiol 75:663-654, 1980.

51. Corabaeuf E, Weldmann S. Potentlel de repos et potentiels d'action du muscle cardlaque me. sures a l'aide d'electrodes internes. C R Soc Bilol 143:1329-1331, 1949.

52. Cosnler $D$, Duchene-Marullaz $P$, Rispat $G$, Strelchenberger $G$. Cardiovascular pharmacology of bepridil ( 1,3 lsobutoxy 2(benzylphenyl)amino propyl pyrrolldine hydrochloride), a new potential ant-anginal compound. Arch Int Fharmacodyn Ther 225:133-151, 1977.

53. Cotoi S, Carasca $E$, Georgescu $C$, Lazar $P$. Two varieties of the ontset of atrial fibrillation studied by monophasic action potential recording. Jpn Heart $J 19: 479-484,1978$.

54. Cramer M, Hartman RJ, Boxer R, Hoffman BF. Electrograms from the canine sinoatrial pacemaker recorded in vitro and in situ. Am $J$ Carcliol 42:939-946, 1978.

55. Cranefield PF. Action potentials, afterpotentlals and arrhythmlas. Circ Res 41:415-423, 1977. 
56. Curry PVL, Evans TR, Krikler DM. Paroxysmal reciprocating sinus tachycardia. Eur J Cardiol $6 / 3: 199-228,1977$

57. Dehtaan RL. Hirakow R. Synchronization of pulsation rates in isolated cardiac myocytes. Exp Cell Res 70:214-220, 1972 .

58. DeHaan RL, Fozzard HA Membrane response to current pulses in spheriodal aggregates of embryonic heart cells. J Gen Physlol 65:207-222, 1975.

59. Deleze J. The recovery of resting potentlial and input resistance in sheep heart injured by kinfe or laser. J Physiol 208:547-562, 1970.

60. Delmar $M$, Jalife $\mathrm{J}$, Michaels DC. Effects of changes in excitability and intercellular coupling on synchronization in the rabblt sino-atrial node. J Physiol 370:127-150, 1986.

61. De Mello WC. The healing-over process in cardiac and other muscle fibers. In: Electrical phenomena in the heart. Ed: De Mello WC. Academic Press, New York, pp 323-351, 1972.

62. De Mello WC. Influence of the sodium pump on intercellular communication in heart fibres: effect of intracellular injection of sodum lon on electrical coupling. J Physiol 263:171-197, 1976.

63. De Mello WC. The Infiuence of $\mathrm{pH}$ on the healing-over of mammalian cardiac muscle. J Physi예 $339: 299-307,1983$

64. Dhingra $R C$, Wyndham $C$, Amat-y-Leon $F$, Denes $P$, Wu D. Rosen KM. Sinus nodal responses to atrial extra stimull in patients without apparent sinus node disease. Am J Cardiol 36:445-452, 1975.

65. Difrancesco D. A study of the lonic nature of the pacemaker current in calf Purkinje fibres. $J$ Physiol 314:377-393, 1981.

66. Draper $\mathrm{MH}$, Weldmann $\mathrm{S}$. Cardiac resting and action potentlals recorded with an intracellular electrode. J Physiol 115:74-94, 1951.

67. Drelfus LS, Michelson EL, Kaplinsky E. Bradyarrhythmias: Clinical significance and management. JACC 1:327-338, 1983.

68. Duchene-Marullaz P, Kantelip JP, Troleze JF. Effects of bepridil, a new antiaginal agent, on ambulatory electrocardlography In human volunteers. J Cardlovasc Pharm 5:506-510, 1983.

69. Ehara T, Kaufmann R. The voltage- and time-dependent effects of $(-)$-verapamil on the slow inward current in isolated cat ventrlcular myocardium. I Pharmacol Exp Ther 207:49-55, 1978.

70. Einthoven W. Ein neues Galvanometer. Ann Physlk vol 4, 12:1059, 1903.

71. Engelimann TW. Ueber das elektrische Verhalten des thätigen Herzens. Arch Ges Physiol $17: 688-99,1878$.

72. Engeimann TW. Onderzoekingen omtrent den oorsprong der normale hartsbeweging en de physlologlsche elgenschappen der grote hartsaderen. Zlttingsverslag der Wis- en Natuurkunde Afdeelling van Junl 1896 aan de koninklijke academie van wetenschappen te Amsterdam. In: Th. W. Engelmann, some papers and his bibllography. Rodopli, Amsterdam, 1984.

73. Erlanger al, Blackman JR. A study of relative rhythmicity and conductivity in various regions of the auricles of the mammalian heart. Am J Physiol 19:125-174, 1907.

74. Eyster JAE, Meek WJ. Experiments on the origin and propagation of the impulse in the heart. 1. The polnt of primary negativity in the mammalian heart and the spread of negativity to other reglons. Heart 5:119-136, 1913-14.

75. Ferrer MII. The sick silnus syndrome in atrlal disease. JAMA 206:645-646, 1968.

76. Ferrer Mll. The slck sinus syndrome. Clrculation 47:635-641, 1973. 
77. Fisch C. Concealed conduction at the AV nodal level. In: Electrophysiology of sinoatrial and at rioventricular nodes. Eds: Mazgalev T, Dreifus LS, Michelson EL. Alan R. LIss" Inc, pp $287-300$, 1988.

78. Flack M. An investigatlon of the sino-auricular node of the mammallan heart. J Physilol $41: 64$ $77,1911$.

79. Flammang $D$, Waynberger $M_{v}$ Jansen FH, Pallet $R$, Coumel P. Electrophysiological profile of bepridil, a new antl-anginal drug with calcium blocking propertles. Eur Heart $J 4: 657-664,1983$.

80. Fleckenstein A. Influence of calcium antagonists on normal calciurn-dependent pacemaker activity. In: Calcium antagonism in heart and smooth muscle. Experimental facts and therapeutic prospects. Ed: Fleckenstein A. John Wiley \& Sons, Inc, New York, pp 165-208, 1983.

81. Garson A, Gillette PC. Electrophysiological studies of supraventricular tachycardia in children. I. Cinical-electrophysiologic correlations. Am Heart J 102:233-250, 1981.

82. Gaskell WH. On the innervation of the heart with special reference to the heart of the tortoise. J Physlol 4:43-127, 1884.

83. Gillmour RF, Zlpes DP. Slow Inward current and cardiac arrhythmias. Am J Cardiol 55:89B$101 B, 1985$.

84. Glomset DJ, Glomset ATA. A morphologic study of the cardlac conduction system in ungulates, $\operatorname{dog}_{n}$ and man. Part I: The sinoatrial node. Am Heart J 20:389-398, 1940.

85. Goldberg JM. Intra-SA-nodal pacemaker shifts induced by autonomlc nerve stimulation in the dog. Am J Physiol 229:1116-1123, 1975.

86. Gomes JAC, Kang PS, Matheson M, Gough WB, El-Sherif N. Coexistence of sick sinus rhythm and atrial flutter-fibrillation. Circulation 63:80-86, 1981.

87. Gomes JAC, Kang PS, El-Sherlf $\mathrm{N}$. The sinus node electrogram in patients with and without sick sinus syndrome: Technique and correlation between directly measured and indirectly estimated sinoatrial conduction time. Circulation 66:864-873, 1982.

88. Gomes $J A C$, Hariman RJ, Chowdry IA. New application of direct sinus node recordings in man: assessment of sinus node recovery time. Circulation 70:663-671, 1984.

89. Gorgels APM, Beekman HM, Brugada P, Dassen WRM, Richards DAP, Wellens HJJ. Extrastimulus-related shortening of the first postpacing interval in digitallis-induced ventricular tachycardla: observations during programmed electrical stimulation in the consclous dog. JACC 1:840-857, 1983.

90. Goto J, Sperelakils $\mathrm{N}$. Depression of automaticlty of the rabbit sa node by bepridlil and nifedipine. Eur J Pharmacol 99:227-231, 1984.

91. Grant AO, Kirkarlan G, Benditt DG, Strauss HC. The estimation of sinoatrial conductlon time in rabbit heart by the constant atrial pacing technique. Circulation 60:597-604, 1979.

92. Griepp EB, Bernfleld MR. Acquisition of synchronous beating between embryonic heart cell aggregates and layers. Exp Cell Res 113:263-272, 1978.

93. Gupta PK, Lichstein E, Chaddla KD, Badur| E. Appralsal of sinus nodal recovery time in patlents with sick sinus syndrome. Am J Cardiol 34:265-270, 1974.

94. Hagiwara $N$, Irlsawa $H$. Contributlon of two types of calcium currents to the pacemaker potentials of sino-atrial node cells. J Physiol 382,104P, 1987.

95. Han J, Malozzl MA, Moe GK. Sinoatrial reciprocation in the Isolated rabblt heart. Circ Res 22:355-362, 1968. 
96. Hariman RJ, Hoffman BF, Naylor RE. Electrical activity from the sinus node region in conscious dogs. Circ Res 47:775-791, 1980.

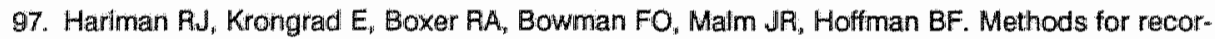
ding electrograms of the sinoatrial node during cardiac surgery in man. Circulation 61:1024$1029,1980$.

98. Hiraoka $M_{1}$ Adaniya $H$. Function of airlal preferential conduction routes under normal and abnormal conditions. J Electrocard lol 16:123-132, 1983.

99. Hoeks APG, Schmltz GML, Allessie MA, Jas $H_{8}$ Hollen SJ, Reneman RS. Multichannel storage and display system to record the electrical activity of the heart. Med Blol Eng Comp 26:434$438,1988$.

100. Hoff HE. Vagal stimulation before the Webers. Ann Med Hist NS 8:138-144, 1936.

101. Horibe $\mathrm{H}$. Studles on the spread of the right atrilal activation by means of intracellular microelectrode. Jpn Circ J 25:583-593, 1961.

102. Irisawa A. Fine structure of the small sinoatrial specimen used for the voltage clamp experiment. In: The sinus node. Structure, function and clinical relevance. Ed: Bonke FIM. Martinus Nijhoff, The Hague, pp 311-319. 1978.

103. Irisawa H. Comparative physlology of the cardlac pacemaker mechanism. Physiol Rev 58:461498,1978 .

104. Irisawa $H_{1}$ Noma A. Pacemaker mechanisms of rabbit sinoatrial node cells. In: Cardiac rate and thythm. Eds: Bouman LN, Jongsma HJ. Martinus Nijhoff, The Hague, pp 35-52, 1982.

105. Irisawa $H$, Hagiwara N. Pacemaker mechanism of mammalian sinoatrial node cells. In: Progress in clinical and biological research vol 275 . Electrophysiology of the sinoatrial and atrioventricular nodes. Eds: Mazgalev T, Dreifus LS, Michelson EL, Alan R. Liss Inc, New York, pp 33-52, 1988.

106. Jallfe $\mathrm{J}_{\text {" Hamilton }} \mathrm{A}_{\mathrm{J}}$, Lamanna $\mathrm{V}$, Moe GK. Effects of current flow on pacemaker activity of the Isolated kilten sinoatrial node. Am J Physiol 238:H307-H316, 1980.

107. Jalife $\mathrm{J}$, Slenter VAJ, Salata $\mathrm{J} J$, Michaels DC. Dynamic vagal control of pacemaker activity in the mammallan sinoatrlal node. Clrc Res 52:642-656, 1983.

108. Jallife $\mathrm{J}$. Mutual entrainment and electrical coupling as mechanisms for synchronous firing of rabbit sino-atrial pace-maker cells. J Physiol (Lond) 356:221-243, 1984.

109. Jallfe $\mathrm{J}$, Michaels $\mathrm{DC}$. Phase-dependent interactions of cardiac pacemakers as mechanisms of control and synchronization in the heart. In: Cardlac Electrophyslology and Arrhythmias. Eds: Zlpes DP, Jalife J. Grune \& Stratton, Oriando, pp 109-119, 1985.

110. Jalife $J_{n}$ Mlchaels DC, Delmar M. Mechanisms of pacemaker synchronization in the sinus node. In: Progress in clinical and blological research vol 275. Electrophysiology of the sinoatrial and atrloventrlcular nodes. Eds: Mazgalev T, Drelfus LS, Michelson EL. Alan R. Liss Inc, New York, pp 67-91, 1988.

111. James TN. Anatomy of the human sinus node. Anat Rec 141:109-139, 1961.

112. James TN, Sherf $L_{n}$ Fine $G$, Morales AR. Comparative ultrastructure of the simus node in man and dog. Circulation 34:139-163, 1966.

113. James TN. Anatomy of the cardlac conduction system in the rabbit. Circ Res 20:638-648, 1967.

114. James TN. The sinus node. Am J cardliol 40:965-986, 1977.

115. James TN. Diversity of histopathologic correlates of atrial fibrillation. In: Atrlal fibrlllation. Eds: Kulbertus HE, Olsson SB, Schlepper M. AB Hässle, Mölndal, Sweden, pp 13-30, 1982. 
116. Jones SB, Euler DE, Hardie $E_{\mathrm{u}}$ Randall WC, Brynjolfsson $\mathrm{G}$. Comparison of $\mathrm{SA}$ nodlall and subsidiary atrial pacemaker function and location in the dog. Am $J$ Physiol 234:H471-H476, 1978.

117. Jongsma $H J$, Masson-Pevet M, Hollander CC, Bruyne de J. Synchronization of the beating frequency of cultured rat heart cells. In: Development and physiological correlates of cardiac muscle. Eds: Lleberman M, Sano T. Raven Press, New York, pp 185-196, 1975.

118. Jongsma $H J$ J, Tsjernina L. Factors influencing regularity and synchronisation of beating of tissue cultured heart cells. In: Cardiac Rate and Rhythm. Eds: Bouman LN, Jongsma HJ. Martinus Niljhoff, The Hague, pp 379-414, 1982.

119. Jongsma $H J$, Tsjernina $L$, Bruine de $J$. The establishment of regular beating in populations of pacemaker heart cells. A study with tissue-cultured rat heart cells. J Moll Cell Cardlol 15:123133, 1983.

120. Jordan ل JL, Yamaguchi I, Mandel WJ, McCullen AE. Comparative effects of overdrive on sinus and subsidlary pacemaker function. Am Heart J 93:367-374, 1977.

121. Jordan JL, YamaguchII, Barrett PA, Mandel WJ. The effects of verapamil on sinoatrial conduction in isolated tissue (Abstr), Clin Ples 26:241, 1978.

122. Jordan JL, Yamaguchl I, Mandel WJ. Function and dysfunction of the sinus node: cillinical studiles in the evaluation of sinus node function. In: The sinus node. Structure, function and cilinlcal relevance. Ed: Bonke FIM. Martinus Nilghoff, The Hague, pp 3-22 "1978.

123. Josephson ME, Caracta AR, Law SH, Gallacher JJ, Damato AN. Electrophysiologlcal evaluation of disopyramide in man. Am Heart J 86:771-780, 1973.

124. Josephson ME, Seides SF. Clinical cardiac electrophysiology: Techniques and interpretations. Lea and Febinger, Philadelphla, p 74, p 187, 1979.

125. Kane KA, Winslow E. Antidysrhythmic and electrophysiologilcal effects of a new antianginal agent, bepridill. J Cardlovasc Pharm 2:193-203, 1980.

126. Kane KA, Berdeja Garcla GY, Sanchez-Perez S, Pastelin G. Electrophysiological effects of IIdocalne, 1 -chlorpheniramine, and bepridil on rabbit sinus node pacemaker cells. J Cardlovasc Pharm 5:102-108, 1983.

127. Karagueuzlan HS, Mandel WJ. The effects of drugs on sinus node function. In: Cardlac electrophyslalogy today. Eds: Masonl A Albonl P. Academic Press Inc. Ltd. London, pp 123-148, 1982.

128. Kass RS, Tsien RW. Multiple effects of caiclum antagonists on plateau currents in cardlac Purkinje fibers. J Gen Physiol 66:169-192, 1975.

129. Kato R. Singh BIN. Effects af beprldil on the electrophyslologle propertles of Isolated canine and rabbit myocardlal fibers. Am Heart J 111:271-279, 1986.

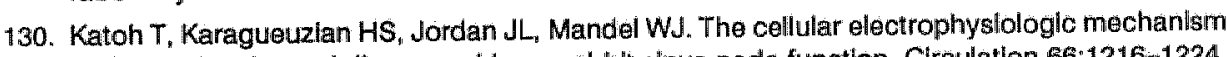
of the dual actions of disopyramide on rabbit sinus node function. Circulation 66:1216-1224, 1982.

131. Kawadla $M$, Satoh $K$, Talra M. Profile of coronary wasodllator and cardiac actions of bepridil revealed by use of Isolated, blood-perfused heart preparations of the dog. J Cardiovasc Pharm 5:604-612, 1983.

132. Kawal $C_{1}$ Konishi $T$, Matsuyama $E_{N}$ Okazaki $H$. Comparative effects of three calcium antagonIsts, diltiazem, verapamil and nifedipline, on the sinoatrlal and atrioventricular nodes. Ex. perimental and clinical studles. Clrculation 63:1035-1042, 1981.

133. Kelth $A$, Flack M. The form and nature of the muscular connections between the primary divlslons of the vertebrate heart. $₫$ Anat Physloll 41:172-189, 1906-07. 
134. Kerr CR, Prystowsky EN, Browning DJ, Strauss $\mathrm{HC}$. Characterization of refractoriness in the sinus node of the rabbit. Circ Res $47: 742-756,1980$.

135. Kerr $\mathrm{CA}$, Strauss $\mathrm{HC}$, Alama $\mathrm{N}$. Effects of basic pacing cycle length on sinus node refractoriness in the rabbil. Am $J$ Cardiol 56:162-167, 1985.

136. Kerr $\mathrm{CR}$. Effect of pacing cycle length and autonomic blockadie on sinus node refractoriness. And Cardlol 62:1192-1196, 1988.

137. Killip T, Gault $\mathrm{JH}$. Mode of onset of atrial fibrillation in man. Am Heart J 70:172-179, 1965.

13B. Keen $\mathrm{HO}$, Singer $\mathrm{DH}_{1}$ Hoffman BF. Effects of atrial premature systoles on sinus rhythm in the rabblit. Circ Res $32: 480-491,1973$.

139. Kobayashi $M$, Shimotorl $M$, Ogiwara $Y$, Chiba S. Effects of calcium channel blockers on sinoatrial conduction in the isolated and blood-perfused dog atrium. Arch Int Pharmacodyn 279:72-82, 1986.

140. Kodamall, Goto J, Ando S, Toyama J, Yamada K. Effects of rapid stimulation on the transmembrane action potentials of rabbit sinus node pacemaker cells. Circ Res 46:90-96, 1980.

141. Kodama $I_{i}$ Boyett MR. Regional differences in the electrical activity of the rabbit sinus node. Pflügers Arch 404:214-226, 1985.

142. Kohlhart $M_{\text {, Flgulla } H} H_{\text {, }}$ Tripathl $O$. The slow membrane channel as the predominant mediator of the excitation process of the sinoatrial pacemaker cell. Basic Res Cardiol 71:17-26, 1976.

143. Kölliker A, Müller $H$. Nachweis der negativen Schwankung des Muskelstroms am natürlich sich kontrahlerenden Muskel. Verhandl Phys Med Ges Wurzburg 6:528-533, 1856.

144. Kreitner $\mathrm{D}$. Evidence for the existence of a rapid sodlum channel in the membrane of rabbit sinoatrial node cells. J Moll Cell Cardiol 7:655-662, 1975.

145. Kreitner $\mathrm{D}$. Electrophysilological study of the twa main pacemaker mechanisms the rabbit sinus node. Cardlovasc Res 19:304-318, 1985.

146. Landmark $\mathbb{K}_{n}$ Amlie $J \mathbb{P}$. A study of the verapamil-induced changes in conductivity and refractorlness and monophasic actlon potentlals of the dog heart in sltu. Eur $\mathrm{J}$ Cardiol 4:419-427, 1976.

147. Lange G. Action of driving stimull from Intrinsilc and extrinsic sources on in situ cardiac pacemaker tissues. Circ Pes 17:449-459, 1965.

148. Langendorf $R$, Lesser ME, Plotkin $P$, Levin $B D$. Atrlal parasystole with interpolation. Observaltlons on prolonged sinoatrial conduction. Am Heart J 63:649-658, 1962.

149. Langendorf A, Pick A, Katz LN. Ventricular response in atrial fibrillation. Role of concealed conduction in the AV junction. Circulation 32:69-75, 1965.

150. Langendorf $\mathrm{A}$. Newer aspects of concealed conductlon of the cardiac impulse. In: The conductlon system of the heart. Structure, function and clinical Implications. Eds: Wellens HJJ, Lie KI, Janse MJ. Stenfert Kroese, Leiden, pp 410-423, 1976.

151. Langendorff $O$, Lehmann $C$. Der versuch von Stannlus am Warmblüterherzen. Pflügers Arch Ges Physlol 112:352-360, 1906.

152. Lowls T. Aurlcular fibrillation: a common clinical condition. Br Med J 2:1528, 1909.

153. Lewis $T$. Auricular flibrillation and its relationship to clinical irregularity of the heart. Heart 1:306$372,1910$.

154. Lewls T. Galvanometrlc curves ylelded by cardlac beats generated in various areas of the aurlcular musculature. The pacemaker of the heart. Heart 2:23-40, 1910. 
155. Lewis T, Oppenheimer BS, Oppenheimer A. The site of origin of the mammalian heart-beat; the pacemaker in the dog. Heart 2:147-196, 1910.

156. Lewis $T$. The pacemaker of the mammallan heart, as ascertained by electrocardlographlc curves. J Physiol (Proc) 39:9-10, 1910-11.

157. Lewis T. Irregularity of the hearts"s action in horses and its relationship to fibrillation of the auricles in experiment and to complete irregularity of the human heart. Heart $3: 161-71,1911-$ 12.

158. Lewis $T$. The pacemaker of the mammalian heart. Trans Int Congr Med London, section 3 , part $1,107-119,1913$.

159. Lewis T, Fell HS, Stroud WD. Observations upon flutter and fibrillation. Part III. Some effects of thythmic stimulation of the auricles. Heart 7:247-292, 1920.

160. Lewis $T$. The mechanism and graphic registratlon of the heart beat. London, Shaw \& Sons, pp $325-343,1925$.

161. Lipsius SL, Vassalle M. Dual excitatory channels in the sinus node. J Mol Cell Cardiol 10:753$767,1978$.

162. Loomls TA, Captain MC, Krop S. Aurlcular fibrillatlon induced and maintained In animals by acem tylcholine or vagal stimulation. Circ Res 3:390-396, 1955.

163. Lowe JE, Hartwich $T$, Takla $M$, Schaper $\downarrow$. Ultrastructure of electrophyslologically identifled human sinoatrial nodes. Basic Res Cardliol 83:401-409, 1988.

164. Lu HH. Shilts In pacemaker dominance within the sinoatrlal reglon of cat and rabbit hearts resulting from increase of extracellular potassium. Circ Res 26:339-346, 1970.

165. Mackaay A.JC. Frequency regulation in the sinus node. Thesis, Rodopl, Amsterdam, 1980.

166. Mackaay AJC, Op 't Hof $T$, Bleeker WK, Jongsma $H J$, Bouman $L N$. Interaction of adrenaline and acetylcholine on cardiac pacemaker function. Functional inhomogeneity of the rabbit slmus node. J Pharmacal Exp Ther 214:417-422, 1980.

167. MacWilliam JA. The mechanism and control of fibrillatlon in the mammalian heart. Proc Roy Soc 90:302-323, 1918.

168. Mandel WJ, Hayakawa $H$, Danzig $R_{n}$ Marcus $H S$. Evaluation of sino-atrlal node function in man by overdive suppression. Cliculation 44:59-66, 1971.

169. Masson-Pevet $M$, Bleeker WK, Mackaay $A J C_{n}$ Gros $D$, Bouman LN. Litrastructure and functlonal aspects of the rabbit sinoatrial node. In: The sinus node, structure, functlon and cllnlcal relevance. Ed: Bonke FIM. Martinus Niljhoff, The Hague, pp 195-211, 1978.

170. Masson-Pevet $M$, Bleeker $W K$, Mackaay $A J C_{n}$ Bouman $L N$. Sinus node and atrium cells from the rabbit heart: a quantitative ellectron microscopic descriptlon after electrophysiologlcal lo. callzation. J Moll Cell Cardiol 11:555-568, 1979.

171. Masson-Pevet $M$, Bleeker WK $K_{n}$ Besselsen E, Treytel BW, Jongsma HJ, Bouman LN. Pacemaker cell types in the rabbit sinus node. A correlative ultrastructural and electrophysiological study. J Moll Cell Cardiol 16:53-63, 1984.

172. Masuda MO, Paes de Carvalho A. Sinoatrial transmission and atrial invasilon during normal rhythm in the rabbit heart. Circ Res $37: 414-421,1975$.

173. Mazgalev T, Drelfus LS, Blanchi J, Michelson EL. Atrioventricular Nodal Conduction Durlng Atral Fibrillation In Rabbit Heart. Am J Physiol 243:H754-H760, 1982.

174. Mazgalev T, Drelfus LS, Iinuma H, Michelson EL. Effects of the Site and Timing of Atrloventricular Nodal Input on Atrioventricular Conductlon In the Isolated Perfused Rabbit Heart. Clrculation 70: 748-759, 1984. 
175. MoWilliam JA. On the rhythm of the mammallan heart. J Physiol 9:167-198, 1888.

176. Mendez $C$, Delmar M. Trlggered activity: tts possible role in cardiac arrhythmlas. In: Cardlac Electrophysiology and Arrhythmias. Eds: Zipes DP, Jalife J. Grune \& Stratton, Orlando, pp 311313,1985

177. Michaels $\mathrm{DC}$, Matyas EP, Jalfie $\rfloor$. Dynamic interactions and mutual synchronization of silnoatrial node pacemaker cells. A mathematical model. Circ Res 58:706-720, 1986.

178. Michaels DC, Matyas EP, Jalife J. Mechanisms of sinoatrial pacemaker synchronization: A new hypothesis. Circ Res 61:704-714, 1987.

179. Michaels DC, Chlalvo DR, Matyas EP, Jalife J. Chaotic activity in a mathematical model of the vagally driven sinoatrial node. Circ Res: (Accepted), 1989.

180. Miller HC, Strauss $\mathrm{HC}$. Measurement of sinoatrial conduction time by premature atrial stimulation in the rabbit. Circ Res $35: 935-947,1974$.

181. Mirro MJ, Watanabe AM, Bailey JC. Electrophysiological effects of disopyramide and quinidine on guinea plg atrla and canine purkinje fibers. Dependence on underlying chollinergic tone. Circ Res 46:660-668, 1980 .

182. Moe GK, Abildskov JA. Atrial fibrllation as a self-sustaining arrhythmla independent of focal discharge. Am Heart لl 58:59-70, 1959.

183. Moe GK. On the multiple wavelet thypothesls of atrial fibrillation. Arch Int Pharmacodyn Ther $140: 183-188,1962$.

184. Moe GK, Rheinboldt WC, Abildskov JA. A computer model of atrial fibrillation. Arn Heart $J$ $67: 200-220,1964$.

185. Moe GK. Computer simulations of atrial fibrillation. In: Computers in blomedical research, wol II. Eds: Stacy RW, Waxman BD. Academic Press, New York, pp 217-238, 1965.

186. Moore EN. Observations on concealed conduction in atrial fibriliation. Circ Res 21:201-208, 1967.

187. Nadeau RA, Roberge FA, Billette J. Role of the sinus node in the mechanism of cholinergic atrial fibrillation. Circ Res 27:129-138, 1970.

188. Nahum LH, Hoff HE. Auricular fibrillation in hyperthyrold patients. JAMA 105:254-257, 1935.

189. Narula OS, Samet $P$, Javier RP. Signiflicance of the sinus-node recovery time. Circulation $45: 140-15 B_{1}, 1972$.

190. Narula OS. SInus node reentry: A mechanism for supraventricular tachycardias. Circulation $50: 11114-1128,1974$.

191. Narula OS, Shantha $N_{\text {, Vasquez }} M_{8}$ Towne WD, Linhart JW. A new method for measurement of sinoatrlal conduction time. Circulation 58:706-714, 1978

192. Nelson JR. Smith JP. Actlivity of the card lac pacemaker of the frog in relation to atrial fibrillation and to other atrlal arrhythmias. Am J Physiol 198:119-122, 1960.

193. Nishi K, Yoshikawa Y, Sugahara K, Morloka T. Changes in electrical activity and ultrastructure of sinoatrlal nodal cells of the rabbits heart exposed to hypoxlc solution. Circ Ries $46: 201-213$, 1980.

194. Noma $A_{a}$ Irisawa $H$. Membrane currents in rabbit sinoatrial node cells studied by the double microelectrode method. Pflugers Arch 364:45-52, 1976.

195. Noma $A_{s}$ Irisawa $H$. Effects of calcium lon on the rising phase of the action potential in rabbit sinoatrlal node cells. Jpn J Physlol 26:93-99, 1976. 
196. Noma A, Yanagthara $K$, Irisawa $H$. lonic currents in rabbit sinoatrial node cells. In. The sinus node, structure, function and dinical relevance. Ed: Bonke FIM. Martinus Nilhoff, The Hague, pp 301-310, 1978 .

197. Ogawa $S$, Dreffus LS, Osmick MJ. Induction of sinus node reentry. its relation to inhomogeneous atrial conduction. J Electrocardiol 11:109-116, 1978.

198. Op 't Hof T, Mackaay AJC, Bleeker W, Jongsma HJ, Bouman LN. Cycle length dependence of chronotropic effects of adrenaline and acetylcholine in the rabbit sinoatrial node. I Autonom Nerv Sys 8:193-204, 1983.

199. Op 't Hof $T$, de Jonge $B$, Mackaay AJC, Bleeker WK, Masson Pevet $M$, Jongsma $H_{4} \downarrow_{4}$ Bouman LN. Functional and marphological organization of the gulnea-pig sinoatrial node compared with the rabbit sinoatrial node. J Mol Cell Cardlol 17:549-564, 1985.

200. Op 't Hof $T$. The mammalian sinus node. A comparative morphological and electrophyslologlcal study. Thesis, Amsterdam, 1986.

201. Op 't Hof $T$, de Jonge $B$, Masson-Pevet $M$, Jongsma HJ, Bouman $L N$. Functional and morphological organlzation of the cat sinoatrial node. J Mol Cell Cardiol 18:1015-1031, 1986.

202. Op 't Hof $T$, de Jonge $B$, Jongsma $H J$, Bouman $L N$. Functional and morphological organization of the pig sinus node. J Mol Cell Cardiol 19:1221-1236, 1987.

203. Orias $\mathrm{O}$, Gilbert $\mathrm{JL}$, Slebens $\mathrm{AA}$, Suckling EE, Brooks CM. Effectiveness of single rectangular electrical pulses of known duration and strength in evoking auricular fibrillation. Am J Physiol $162: 219-225,1950$.

204. Paes de Carvalho A, De Mello WC. Hoffman BF. Electrophysiologlcal evidence for speclalized fiber types in rabbit atrium. Am J Physiol 196:483-488, 1959.

205. Paes de Carvalho A. Celitular electrophysiology of the atrial speciallzed tissues. In: The speclallized tissues of the heart. Eds: Paes de Carvalho A, De Mello WC, Hoffman BF. Elsevier, New York, pp 115-133, 1961.

206. Pahlajani DB, Miller RA, Serratto M. Sinus nóde reentry and silnus node tachycardla. Am Heart J 90:305-311, 1975.

207. Paritzky $Z$, Obayashi $K$, Mandel WJ. Atrial tachycardla secondary to sino-atrial node reentry. Chest 66:526-529, 1974.

208. Paculay KL, Varghese $P J$, Damato AN. Sinus node reentry. An in vilvo demonstration in the dog. Circ Res 32:455-463, 1973.

209. Paulay KL, Ruskin JN, Damato AN. Sinus and atrioventricular nodal reentrant tachycardla in the same patlent. Am J Cardlol 36:810-816, 1975.

210. Prinzmetal M, Rakita L, Borduas JL, Flamm $E$, Goldman A. The nature of spontaneous auricular fibrillation in man. With comments on the action of antlarrhythmic drugs. JAMA 157:1175$1182,1955$.

211. Prystowsky EN, Grant $A O$, Wallace $A G$, Strauss $H C$. An analysls of the effects of acetylcholline on conduction and refractoriness in the rabbit sinus node. Circ Res 44:112-120, 1979.

212. Prystowsiky EN. The sick sinus syndrome - Dlagnosis and treatment. In: Advances and controversles in cardlology. Ed: Donoso E. Thleme-Stratton linc. pp 93-105, 1981.

213. Relffel JA, Bigger JT. Current status of direct recordings of the sinus node electrogram in man. PACE 6:1143-1150, 1983.

214. Reiffel JA, Gang E, Livelli FD, Gliktich J, Blgger JT. Indirectly estimated sinoatrial conduction time by the premature atrial stimulatlan technique: patterns of error and degree of association In accuracy as assessed by direct sinus node electrography. Am Heart J 106:459-463, 1983. 
215. Reiffel JA, Bigger JT, Ferrick K, Livell FD, Gilklich J, Wang P, Bosner R. Sinus node echoes and concealed concealed conduction: additional sinus node phenomena confirmed in man by direct sinus node electrography. J Electrocardiol 18:259-266, 1985.

216. Reiffel JA. Clinical electrophysidogy of the sinus node in man. In: Progress in cilinical and biologlcal research vol. 275. Electrophysiology of the sinoatrial and atrioventricular nodes. Eds: Mazgalev T, Dreifus LS, Michelson EL. Alan R. Liss Inc, New York, pp 239-257, 1988.

217. Reln Aul JT, Simcha A, Ludomirsky A, Appelbaum A, Uretaky $G$, Tamir I. Symptomatic sinus bradycardla in infants with structurally normal hearts, J Pediatr 107:724-727, 1985.

218. Fosen MF, Danilo P. Effects of tetrodotoxin, lidocaine, verapamil and AHR-2666 on ouabain induced detayed afterdepolarizations in camine Purkinje fibers. Circ Res 46:117-124, 1980.

219. Rosenblueth A, Garcia Ramos J. Studies on flutter and fibrillation II. The influence of artificial obstacles on experimental auricular flutter. Am Heart $\mathrm{J}$ 33:677-684, 1947.

220. Posenblueth $\mathrm{A}$. The mechanism of auricular flutter and auricular fibrillation. Circulation 7:612$613,1953$.

221. Powland E, McKenna WJ Krikler DM. Electrophysiologic and antlarrhythmic actlons of bepridil. Comparison with verapamil and ajmalline for atrioventricular reentrant tachycardia. Am J Cardiol $55: 1513-1519,1985$.

222. Aubenstein DS, Lipsilus SL. Mechanisms of automaticity in subsidiary pacemakers from cat right atrlum. Clrc Res 64:648-657, 1989.

223. Sanderson JB, Page FJM. On the time-relations of the excitatory process in the ventricle of the theart of the frog. J Physiol 2:384-435, 1879-80.

224. Sanderson JB, Page FJM. On the electrical phenomena of the excitatory process in the heart of the frog and of the tortoise, as investigated photographically. J Physiol 4:327-338, 1883-4.

225. Sano T, Yamagishi S. Spread of excitation from the sinus node. Circ Fes 16:423-430، 1965.

226. Sano $T$, Suzukl $F$, Sadayukl S. Sinus Impulses and atrial fibrillation. Circ Res 21:507-513, 1967.

227. Sano $T$, llida $Y$. The sinoatrial connectlion and wandering pacemaker. d Electrocardiol 1:147$153,1968$.

228. Sano T, Sawanoborl T. Adaniya $\mathrm{H}$. Mechanisms of rhythm determination among pacemaker cells of the mammallan sinus node. Am J Physlol 235:H379-H3B4, 1978.

229. Sassine A, Masse C, Faluri A, Hirsch J, Labrid C, Puech P. Electrophysiologic effects of bepridil In the anesthetized dog studied by endocardiac electrocles. Am J Cardlol 53:1707-1711, 1984.

230. Sasynluk $\mathrm{BI}$, Mendez $\mathrm{C}$. A mechanism for reentry in canine ventricular tissue. Circ Res 28:3-15, 1971.

231. Satake S, Blanchi J, Dreifus LS. Conduction disturbances in the middle internodal tract of the rabbit heart. J Electrocardiol 14:371-378, 1981.

232. Scherf D, Romano FJ, Terranova R. Experimental studies on auricular flutter and auricular fibrillation. Am Heart J 36:241-251, 1948.

233. Scherf D. Terranova F.. Mechanlism of aurlcular flutter and fibrillation. Am J Physiol 159:137142, 1949.

234. Scherf $D$, Chlck FB. Abnormal cardlac rhythrns caused by acetylcholine. Circulation 3:764-769, 1951.

235. Scherf D, Schaffer Al, Blumenfeld S. Mechanism of flutter and fibrillation. Arch Int Med 91:333$352,1953$. 
236. Scherf D. The atrial arrhythmias. N Eng J Med 252:928-933, 1955.

237. Schreurs AW, Meljer AA used for microelectrode work. Pflügers Arch 346:163-166, 1974

238. Schwartz $A$, Matib MA, Balwierczak $J_{"}$ Lathrop DA. Pharmacology of calclum antagonists. Am $\checkmark$ Cardiol $55: 3 \mathrm{C}-7 \mathrm{C}, 1985$.

239. Schweitzer $P$, Mark $H$. The values and limitations of deductive analysis and electropliyslological testing in patients with sinoatrial arrhythmias. Pace $7: 403-420,1984$.

240. Senges J, Mlzutani T, Pelzer D, Brachmann J, Sonnhof U, Kabler W. Effect of hypoxila on the sinoatrial node, atrium and atroventricular node in the rabbit heart. Circ Res 44:856-863, 1979.

241. Seyama I. Effect of high calcium concentration on the action potential of the skate heart. Am J Physiol 216:687-692, 1969.

242. Seyama 1 . Characteristics of the rectifying properties of the sinoatrial nodle cell of the rabblt. $J$ Physiol 255:379-397, 1976.

243. Shimuzu K. Various aspects of atrial beats observed in the dogs with Injured sino-atrial and atrloventrlcular node and a correlation between these conditions and aconitine produced atrial fibrillatlon. J Tokyo Med Coll 25:277-299, 1967.

244. Singh BN, Baky S, Nademanee K. Second-generation calcium antagonists: search for greater selectivity and versatility. Am J Cardiol 55:214B-221B, 1985.

245. Singh BN, Nademanee $K$, Feld G, Plontek $M_{n}$ Schwab $M$. Comparative electrophysiologilo profiles of calclum antagonists with partlcular reference to bepridil. Am J Cardiol 55:14C-19C, 1985.

246. Slenter VAJ, Salata $\mathrm{JJ}$, Jallíe $\mathrm{J}$. Vagal control of pacemaker periodiclty and intranodal conduction in the rabbit sinoatrial node. Circ Res 54:436-446, 1984.

247. Smeets JLRM, Allessie MA, Lammers WJEP, Bonke FlM, Hallen SJ. The wave length of the cardiac impulse and reentrant arrhythmias in isolated rabbit atrium. The role of heart rate, auton. omlc transmitters, temperature and potasslum. Circ Res 58:96-108, 1986.

248. Spear JF, Kronhaus KD, Moore EN, Kline RP. The effect off brief vagal stimulation on the isolated rabblt sinus node. Circ Res 44:75-87, 1979.

249. Stelnbeck G, Allessie MA, Bonke FIM, Lammers WJEP. Sinus node response to premature atrlal stimulation in the rabbit studied with multiple microelectrode impaliements. Clrc Aes 43:695704, 1978.

250. Stelinbeck G, Bonke FIM, Allessie MA, Lammers WJEP. The effects of outabain on the lsolated sinus node preparation of the rabbit studled with microelectrodes. Circ Fes 46:406-414, 1980.

251. Steinbeck $G$, Habert $R_{x}$ Luderitz B. Effects of atrial pacing on atrio-sinus conduction and overdrive suppression in the isolated rabbit sinus node. Circ Res 46:859-869, 1980.

252. Stelnman RT, Lehmann MH. Beat-to-beat changes in atrioventricular nodal excltablilty and its modulation by concealed conductlon during functional 2:1 block in man. Circulation 76, 759$767,1987$.

253. Strauss $\mathrm{HC}_{\mathrm{v}}$ Blgger JT. Electrophysiological propertles of the rabbit perinodal flbers. Circ Res 31:490-506, 1972.

254. Strauss HC, Saroff AL, Blgger JT, Glardina EGV. Premature atrial stimulation as a key to the understanding of sinoatrlal conduction In man. Presentation of data and critical revlew of the literature. Circulation 47:86-93, 1973. 
255. Strawss HC, Bigger JT. SaroH AL, Giardina EV. Electrophysiologic evaluation of sinus node function in patlents with sinus node dysfunction. Circulation 53:763-776, 1976.

256. Strauss $\mathrm{HC}_{\text {" }}$ Geer MR. Sinoatrial reentry. In: Reentrant Arrhythmias. Mechanisms and treatment. Ed: Kulbertus: HE MTP Press Ltd, Lancaster, pp 27-38, 1977.

257. Strauss HC, Schelnman MM, LaBarre A, Browning DJ, Wenger $T L$, Wallace AG. Review of the silgniflcance of druggs in the sick sinus syndrome. In: The sinus node. Structure, function and clinical relevance. Ed: Bonke FIM. Martinus Nijhoff, The Hague, pp 103-111, 1978.

258. Sutyagin PV. Pylaev AS. Iclentification of sinus node pacemaker cells of the heart by intracellular Injection of lanthanum lons. Buil Exp Blol Med 95:680-682, 1983.

259. Talra N. Verapamil, dlitazern, mifedipine - comparative studlies on the AV node. In: New calcium antagonists recent developments and prospects. Drug Development and Evaluatlon 9. Eds: Fleckenstein $A_{\text {, Hashimoto }} K_{n}$ Herrmann M, Schwartz A, Seipel L. Gustav Fischer Verlag, Stuttgart, New York, pp 37-52, 1983.

260. Takayanagl $K$, Jalfe $J$. Effects of digitalis intoxication on pacemaker rhythm and synchronization in the rabbit sinus node. Am J Physiol 250:H567-H578, 1986.

261. Taussig HB. On the boundaries of the sino-auricular node and the atrio-ventricular node in the human heart. Bull Johns Hopkins Hosp 48:162-178, 1931.

262. Taylor $\mathrm{J} J, D^{\prime}$ Agrosa $L S$, Burns EM. The pacemaker cell of the sinoatrial node of the rabbit. Am J Physlol 235(4):H407-H412, 1978.

263. Ten Elck RE $E_{n}$ Nawrath $H$, McDonald TF, Trautwein $W$. On the mechanism of the negative inotroplc effect of acetylcholine. Pflügers Arch 361:207-213, 1976.

264. Toda N, Shimamoto $K$. The Influence of sympathetic stimulation on transmembrane potentials In the S-A node. J Pharmacol Exp Ther 159:298-305, 1968.

265. Toda N. Electrophyslologlcal effects of potassium and calcium ions in the sino-atrial node in response to sympathetic nerve stimulation. Pflugers Arch 310:45-63, 1969.

266. Tranum-Jensen J. The fine structure of the sinus node: a survey. In: The sinus node. Structure, function and cllnical relevance. Ed: Bonke FIM. Martinus Nijhoff, The Hague, pp 149-165, 1978.

267. Trautweln $W_{n}$ Zlnk K. Ueber Membran- und Aktionpotiale einzelner Myokardfasern des Kaltund Warmblutterherzens. Pfluggers Arch Ges Physiol 256:68-84, 1952.

268. Trautweln W. Uchlzono K. Electron microscoplc and electrophyslologic study of the pacemaker in the sinoatrial node of the rabbit heart. Zelt Zellforsch Mikrosk Anat 61:96-109, 1963.

269. Trltthart HA, Koldl B. Dltiazem effects on cardlac Impulse generation. In: New calcium antagonists recent developments and prospects. Drug Development and Evaluation 9. Eds: Flecken-

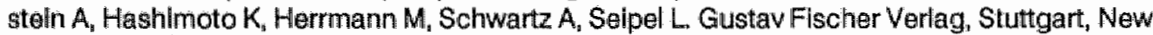
York, pp 53-62, 1983.

270. Truex RC. Comparative anatomy and functional considerations of the cardiac conduction system. In: The speclallzed tissues of the heart. Eds: Paes de Carvalho $A_{1}$ De Mello WC, Hoffman BF. Elsevler, New York, pp 22-43, 1961.

271. Van Capelle FJL, Durrer D, Computer simulations of arrhythmlas in a network of coupled excitable elements. Circ Res 47:454-466, 1980.

272. Vassalle M. The relationshlp among cardlac pacemakers. Overdrive suppression. Circ Res 41:269-277, 1977 .

273. Vassalle M. Electrogenesls of the plateau and pacemaker potential. Ann Rev Physlol 41:425$440,1979$. 
274. Vaughan Williams EM. Disopyramide. In: Clinical pharmacology of cardiac antiarrhythmic agents: classical and current concepts reevaluated. Ed: Garfein $O B$, Annals of the New York academy of sclences, vol 432, pp 189-200, 1984.

275. Vaughan Williams EM. A classification of antiarrhythmle actlons reassessed after a decade of new drugs. J Clin Pharmacol 24:129-147, 1984.

276. Veenstra RD, DeHaan RL. Electrotonic interactions between aggregates of chick embryo car. diac pacemaker cells. Am J Physiol 250:H453-H463, 1986.

277. Vogel S, Crampton R, Sperelakis N. Blockade of myocardlal slow channels by bepridil (CERM 1978). J Pharmacol Exp Ther 210;378-385, 1979.

278. Von Haller A. Dr. Albert Von Haller's Physiology: Being a course of lecture upon the visceral anatomy and vital oeconomy of human bodies. Vol 1, London, 1754.

279. Wallace $A G$, Daggett WM. Reexcitation of the atrium. The echo phenomenon. Am Heart J 68:661-666, 1964.

280. Waller $A D$. A demonstration on man of the electromotive changes accompanying the heart's beat. J Physilol 8:229-234, 1887.

281. Watanabe $Y$, Dreifus LS. Newer concepts in the genesis of cardiac arrhythmias. Am Heart J 76:114-135, 1968.

282. Watanabe $Y$, Nishimura $M$, Yamadla S. Significance of a combination of different experimantal approaches. Heart 16:513-523, 1984.

283. Weingart $R$. The actions of ouabain on Intercellular coupling and conductlon vellocity in mammalian ventrlcular muscle. J Physiol 264:341-365, 1977.

284. Welsfogel GM, Batsford WP, Paulay KL, Josephson ME, Ogunkelu JB, Akhtar M, Seides SF, Damato AN. Sinus node re-entrant tachycardla in man. Am Heart J 90:295-304, 1975.

285. Wellens HJJ. Role of sinus node re-entry in the genesis of sustained cardlac arrhythmlas. In: The sinus node. Structure, function and clinical relevance. Ed: Bonke FIM. MartInus Nijhoft, The Hague, pp 422-427, 1978 .

286. West TC. Ultramicroelectrode recording from the cardiac pacemaker. J Pharmacol Exp Ther 115:283-290, 1955.

287. West TC, Falk G, Cervonl P. Drug alteration of transmembrane potentials in atrial pacemaker cells. J Pharmacoll Exp Ther 117:245-252, 1956.

288. Wit $\mathrm{AL}$, Cranefleld $\mathrm{PF}$. Effect of verapamil on the sinoatrial and atrioventricular nodles of the rabbit and the mechanism by which it arrests reentrant atrloventrlcular nodal tachycardia. Circ Res $35: 413-425,1974$.

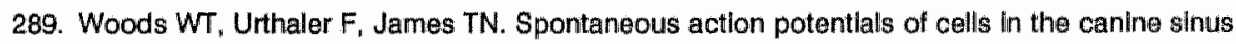
node. Circ Res 39:76-82, 1976.

290. Wu D, Amat-y-Leon F, Denes P, Dhingra RC, Pletras RJ, Rosen KM. Demonstration of sustained sinus and atrlal reentry as a mechanism of paroxysmal supraventrlcular tachycardia. Clrcullation 51:234-243, 1975.

291. Yabek SM, Swensson RE, Jarmakani JM. Electrocardiographic recognition of sinus node dysfunctlon in children and young adults. Circulation 56:235-239, 1977.

292. Yamagishi $S$, Sano $T$. Effect of tetrodotoxin on the pacemaker action potential of the sinus node. Proc Jpn Acad 42:1194-1196, 1966.

293. Yanagihara $K$, Irlsawa $H$. Inward current actlvated during hyperpolarlization in the rabbit sinoatrial node cell. Pflügers Arch 385:11-19, 1980. 
294. Yanagihara $\mathrm{K}$, Irisawa $\mathrm{H}$. Potassium current during the pacemaker depolarization in the rabbit sincaitral node cell. Pflugers Arch 380:255-260, 1980.

295. Ypey DL, Clapham DE, DeHaan FL. Development of electricall coupling and action potential synchrony batween palred aggregates of embryonic heart cells. J Membr Biol 51:75-96, 1979.

296. Zpes DP. Fischer JC. Effects of agents which inhibit the slow channel on sinus node automaticlty and atroventricular conduction in the dog. Circ Res 34:184-192, 1974 . 


\section{SUMMARY}

In the isolated rabbit heart we studied the passive electrical influences of the atrial myocardium on sinus function (chapter II). It was found that the atrial myocardium causes an electrotonic depression of spontaneous diastolic depolarization in fibers in the border zone of the sinus node. This explains the origination of the sinus impulse from fibers in the center of the sinus node, although fibers in the sino-atrial border zone possess equivalent intrinsic pacemaker properties. From the nodal center the impulse is transmitted through the border zone of the sinus node towards the atrial myocardium. Using a new model we studied the basic conduction characteristics of the sinus node and the effects of several environmental changes (chapter III). It was found that impulse conduction accelerates and refractoriness shortens from the center of the sinus node towards the crista terminalis. During slow heart rates the conduction velocity and effective refractory period in the border zone were $7-11 \mathrm{~cm} / \mathrm{s}$ and $105 \mathrm{~ms}$, and in the center of the sinus node $2-5 \mathrm{~cm} / \mathrm{s}$ and $165 \mathrm{~ms}$ respectively. The conduction properties of the sinus node were markedly depressed by hypothermia, moderate hypoxia and acetylcholine administration. Norepinephrine increased the conduction velocity and shortened the refractory period in the sinus node. The present model was also used to study the effects of four antiarrhythmic drugs on sinus function (chapters IV and V). Bepridil and verapamil predominantly depressed conduction and refractoriness in the sinus node, prior to any effect on sinus automaticity or atrial conduction properties. Diltiazem on the other hand, depressed sino-atrial conduction and sinus rate to a similar extent. Disopyramide mainly depressed the atrial conduction properties although with higher drug concentrations conduction through the sinus node deteriorated as well.

Sinus function during atrial fibrillation was studied by means of extracellular mapping of the atrium and intracellular recordings from the sinus node. We found that during atrial fibrillation the sinus node was invaded by multiple fibrillatory wavelets coming from different directions and with an average interval of about $80 \mathrm{~ms}$ (chapter VI). However, impulses also emerged from the sinus node (about once per second) and participated in the fibrillatory process. This phenomenon increased the average number of atrial wavelets during fibrillation. Intracellular recordings revealed a progressive sino-atrial entrance block protecting the pacemaker fibers in the center of the sinus node against the high rate of penetrating atrial impulses (chapter VII). As a result, the pacemaker fibers showed an activation rate far below their maximal response rate during regular pacing and only slightly faster than normal sinus rhythm. Spontaneous diastolic depolarization still developed and incidentally led to spontaneous impulse formation during atrial fibrillation. However, the high activation rate in the sino-atrial border zone prevented these spontaneous impulses to exit 
from the sinus node. On the other hand, the frequent occurrence of local sino-atrial conduction block favoured sinus reciprocation (sinus echo beats) and strongly suggested this mechanism to underly the origination of activation waves from the sinus node during fibrillation. Additional evidence for the occurrence of sinus echo beats was provided by the bimodal distribution of the first post-fibrillatory escape interval (chapter ViII). This bimodality suggested that the first sinus escape beat after spontaneous termination of atrial fibrillation could be based on two different mechanisms. Relatively late escape beats represented spontaneous impulses from the sinus node; early escape beats (25\%) were probably based an sinus reciprocation of one of the last fibrillatory wavelets. It was concluded that the sinus node might facilitate the perpetuation of atrial fibrillation and easily restart this arrhythmia by the frequent occurrence of sinus echo beats. The limited degree of sinus overdrive suppression caused a prompt sinus recovery after spontaneous termination of atrial fibrillation. 


\section{SAMENVATTING}

Aan het begin van elke hartslag staat de vorming van een elektrische prikkel in de sinusknoop, een structuur gelegen in de wand van de rechter boezem van het hart. Deze prikkel wordt vervolgens snel voortgeleid door de wand van beide boezems en kamers van het hart met als gevolg dat ceze compartimenten gecoördineerd samentrekken en het bloed door de kleine en grote lichaamscirculatie pompen. In het huidige proefschrift zijn de resultaten beschreven van een aantal experimentele studies waarin de vorming en voortgeleiding van de elektrische prikkel in de sinusknoop zelf centraal staan, en waarin het gedrag van de sinusknoop tijjens boezemfibrilleren uitgebreid is onderzocht.

Hoofdstuk \& geeft een algemene inleiding in de sinusknoop electrofysiologie en aanverwante ritmestoornissen van het hart. Hoofdstuk II beschrijft hoe de impulsvorming in de sinusknoop beinvloed wordt door de koppeling aan het omringende boezemweefsel. in het geïsoleerde sinusknoop preparaat van het konijnehart resulteerde chirurgische scheiding van boezem- en sinusknoop weefsel in een plotselinge versnelling van het spontane sinusritme $(+20 \%)$. Reconstructie van het elektrisch activatiepatroon in de sinusknoop liet zien dat deze versnelling gepaard ging met een verschuiving van de plaats van oorsprong van de impuls van het centrum naar de rand van de sinusknoop. Kwantificering van de actie potentiaal karakteristieken in het sinusknoop gebied wees uit dat onder normale omstandigheden de spontane impulsvorming in de randcellen electrotoon wordt onderdrukt door het aangrenzende boezemweefsel. Het gevolg van deze onderdrukking is dat de sinus impuls normaliter in het centrum van de geïsoleerde sinusknoop onstaat, buiten de invloedssfeer van het boezemweefsel. Op grand van deze bevindingen concludeerden wij dat de cellen in de rand en het centrum van de sinusknoop gelijkwaardige intrinsieke pacemaker eigenschappen bezitten.

In hoofdstuk III komt de voortgeleiding van de impuls binnen de sinusknoop aan de orde. De basale geleidings eigenschappen in het centrum en de rand zone van sinusknoop werden bestudeerd met behulp van een nieuw onderzoeksmodel. Hierbij werd het geisoleerde prepareat zodanig gemodificeerd dat twee delen boezemweefsel enkel en alleen verbonden waren door de sinusknoop. Aldus kon de geleidingstijd van gestimuleerde atriale impulsen door de sinusknoop eenvoudig gemeten worden. Uit deze experimenten bleek dat vanaf het centrum van de sinus knoop naar het boezemweefsel de geleidingssnelheid van de elektrische impuls toeneemt en de refractaire periode korter wordt. Bij trage hartritmes bedroeg de geleidingssnelheid en de effectieve refractaire periode in de rand van de sinusknoop respectievelijk $7-11 \mathrm{~cm} / \mathrm{s}$ en $105 \mathrm{~ms}$, en in het centrum respectievelijk $2-5 \mathrm{~cm} / \mathrm{s}$ en 
$165 \mathrm{~ms}$. De geleidings eigenschappen in de sinusknoop werden aanzienlijk onderdrukt door hypothermie, hypoxie en acetylcholine. De geleidingssnelheid nam af terwill de refractaire periode langer werd. Alleen noradrenaline veroorzaakte een verhoging van de geleidingssnelheid en een verkorting van de refractaire periode. Het huidige onderzoeksmodel werd toegepast am de effecten van een viertal geneesmiddelen op het functioneren van de sinusknoop te onderzoeken. De resultaten van deze studies zijn beschreven in hoofdstuk IV en V. Zowel bepridil als verapamil onderdrukten met name de geleidings eigenschappen van de sinusknoop zonder noemenswaardige invloeden uit te oefenen op de frequentie van prikkelvorming (sinusritme). Diltiazem veroorzaakte zowel een vertraging van het sinusritme als een verlaging van de voortgeleidingssnelheid en verlenging van de refractiare periode in de sinusknoop. Deze effecten waren vrijwel even sterk. Disopyramide daarentegen onderdrukte met name de geleidings eigenschappen van het boezemweefsel. Hogere concentraties van dit farmacon veroorzaakten echter ook een vertraging van de impulsgeleiding in de sinusknoop. Deze experimenten demonstreerden dat antiaritmica, ook van dezelfde klasse, op velerlei wijzen het functioneren van de sinusknoop kunnen beiinvloeden. Het effect van een farmacon op het sinusritme bleek geen representatieve maat voor het totale effect op het functioneren van de sinusknoop.

In het Langendorff geperfundeerde konijnehart werd de rol van de sinusknoop tijdens boezemfibrilleren uitgebreid onderzocht. De resultaten van deze studie zijn beschreven in de hoofdstuk VI, VII en VIII. Met behulp van een probe, ingebracht in de rechter boezemholte en voorzien van 248 unipolaire oppervlakte-electrodes, werd het elektrisch activatie patroon in de wand van de rechter boezem tijdens boezemfibrilleren gereconstrueerd (hoofdstuk VI). Uit deze experimenten bleek dat de sinusknoop tijdens boezemfibrilleren voortdurend (gemiddeld interval $80 \mathrm{~ms}$ ) en van alle kanten wordt bestookt door activatiegolven. Daarnaast echter werden ook regelmatig (gemiddeld ongeveer 1 maal per seconde) activatiegolven geïdentificeerd die ontsprongen aan de rand van de sinusknoop. Deze sinus impulsen namen deel aan het fibrillatie-proces en vergrootten zo wellicht het gemiddeld aantal onafhankelijke activatiegolven tijdens boezemfibrilleren. Met behulp van intracellulaire afleidingen werd de lokale electrische activiteit in de sinusknoop zelf bestudeerd. Registraties op verschillende plaatsen in de sinusknoop lieten zien dat in de rand van de sinusknoop een toenemende graad van geleidingsblok bestond tijdens boezemfibrilleren (hoofdstuk VII). De gemiddelde activatie-frequentie van de randcellen bedroeg slechts de helft van de fibrillatie-frequentie in het boezemweefsel. Door dit sino-atriaal geleidingsblok vertoonden de cellen in het centrum van de sinusknoop zelfs een gemiddeld activatieritme dat nauwelijks sneller was dan het normale sinusritme en veel trager dan het maximale activatieritme van deze cellen. Dientengevolge 
ontwikkeiden de pacemaker cellen in het centrum van de sinusknoop nog steeds spontane diastolische depolarizatie, hetgeen regelmatig leidde tot spontane impulsvorming in de sinusknoop tijdens boezemfibrilleren. Deze spontane impulsen konden het ontstaan van golffronten aan de rand van de sinusknoop tijdens boezemfibrilleren echter niet verklaren. De hoge graad van geleidingsblok in de rand van de sinusknoop belette de spontane impulsen immers het centrum van de sinusknoop te verlaten.

Een meer voor de hand liggende verklaring voor het ontstaan van activatiefronten aan de rand van de sinusknoop was het optreden van sinusknoop echo slagen ten gevolge van een unidirectioneel geleidingsblok tussen boezem en sinusknoop. Intracellulaire afleidingen op de overgang van boezem naar sinusknoop be-vestigden het frequente optreden van lokaal sino-atriaal geleidingsblok. Het herstel van het sinusritme na spontaan termineren van boezemfibrilleren wees eveneens sterk op het optreden van sinusknoop echo's tijdens boezemfibrilleren (hoofdstuk VIII). Een spontaan gestopte episode van boezemfibrillatie werd dikwills gevolgd door een relatief vroege spontane prikkel uit de sinusknoop. Kwantificering van het postfibrillatoir sinusritme in een groot aantal gevallen leverde een bimodale verdeling op van het tijdsinterval tussen de laatste fibrillatieslag en de eerste sinusknoop impuls. In $25 \%$ van de gevallen bleek deze eerste sinusknoop activatie veel $(-55 \%)$ vroeger te komen dan verwacht op grond van het sinusritme voorafgaand aan het fibrilleren. Deze bimodale verdeling wees sterk op het bestaan van twee verschillende mechanismen voor het onstaan van de eerste post-fibrillatoire sinusknoop impuls. Daar de relatief lange 'escape'-intervallen 'gebaseerd waren op normale spontane impulsvorming in de sinusknoop, was het zeer waarschijnlijk dat de korte "escape"-intervallen gebaseerd waren op een sinusknoop echo slag die voortvloeide uit een van de laatste fibrillatiegolven. Uit deze experimenten bleek tevens dat boezemfibrilleren slechts een geringe 'overdrive suppressie' uitoefend op de sinusknoop. Op grond van de huidige resultaten concluderen wij dat de sinusknoop naar aller waarschijnlijkheid bijdraagt aan de in-stand-houding van boezemfibrilleren door het frequente optreden van sinusknoop echo's. Deze echo's kurnen tevens het spontaan getermineerde fibrillatie-proces gemakkelijk herstarten. De geringe mate van sinusknoop 'overdrive suppressie' en het optreden van verborgen impulsvorming tijdens fibrilleren maken dat het sinusritme zich na het spontaan stoppen van boezemfibrilleren vrijwel onmiddellijk hersteld. 


\section{NAWOORD}

Het schriven van een proefschrift is lang geen sinecure. In de tijd die eraan vooraf gaat en waarin het promotie onderzoek gestalte krijgt zie je vaak hoe 'terminale' promovendi hectisch door het biomedisch centrum lopen, opgejaagd als jonge honden om alles op tijd klaar te krijgen: eerst het manuscript voor de beoordelingscommissie, daarna het origineel voor de drukker. Telkens wanneer ik hen zag of van dichtbij meemaakte dacht ik: 'dit zal mij niet gebeuren, ik begin ruimschoots op tijd met schrijven". Maar helaas, ook ik ben aan die enorme stress en tijdsdruk ten prooi gevallen. Tijdsplanningen kwamen ineens niet meer uit, na een lange werkdag en -nacht had ik nog steeds geen voldaan gevoel, en voor mijn directe omgeving werd ik onuitstaanbaar. Achteraf gezien blijkt deze toestand inherent te zijn aan het schrijven van een proefschrift, en is het irreeeel om te denken dat het in jouw geval anders zall verlopen dan bij je voorgangers. Het was juist deze stress die mij maandenlang op de been hield. Maar dat was niet het enige. Aan mijn opleiding, thet welslagen van alle experimenten, het uitwerken van de vele gegevens en het verschijnen van dit proefschrift in zijn uiteindelijke vorm droegen ook anderen bij. Mijn dank! Een aantal mensen hebben hierin echter een bijzonder aandeel gehad en hen wil ik dan ook uitdrukkelijk bedanken:

Allereerst mijn beide promotoren.

Mijn intrede en opleiding in de sinusknoop fysiologie heb ik voornamelijk aan mijn promotor Prof. F.I.M. Bonke te danken. Beste Vic, in de prilste jaren van mijn wetenschappelijke loopbaan was jij het die milj op zeer persoonlijke en bijna vaderlijke wijze begeleidde, de kneepjes van het vak bijbracht en mijn enthousiasme voor de wetenschap grondig aanwakkerde. Je hield me vaak experimentele hoogstandjes voor die je in dezelfde ademteug bestempelde als "...uiterst moeilijk" of "...dat lukt je nooitl", wetende dat ik dit soort uitdagingen niet kon weerstaan. Dat je ooit met één tik op de experiment-tafel een microelectrode impaleerde nadat ik reeds anderhalf uur vruchteloze pogingen hiertoe had ondernomen zal ik niet snel vergeten. De keren dat ik dit - overigens zonder succes - heb geprobeerd te evenaren zijn ontelbaar. Ik ben ermee opgehouden toen ik met één tik kon expaleren. Mijn frustratiedrempel was toen echter zover gestegen dat ij van lieverlede maar rector magnificus bent geworden, behalve dan op die nostalgische woensdagen.

Mijn huidige leermeester en promotor Prof. M.A. Allessie. Beste Maurits, de vriendschappelijke en leerzame samenwerking met jou zoals die nu reeds enkele jaren voortduurt is voor mij een permanente bron van motivatie en uitdaging. Motivatie wanneer je weer eens nieuwe ideeën ventileert die mijn geduld danig op de proef 
stellen wanneer we ze niet meteen tot litvoering kunnen brengen; een uitdaging is het trachten te evenaren van jouw nimmer aflatende drang naar perfectie, zowel in experimenteel als publicerend opzicht, en je stapsgewijze aanpak bil het oplossen van problemen. Bovenal staat echter het saamhorigheidsgevoel dat jij voortdurend binnen de groep elektrofysiologie weet te handhaven. Het is jammer dat je zelf weinig gelegenheid meer krijgt om samen met ons leuke 'proefjes' te doen. Maar wanneer je dan toch een enkele keer kunt meedraaien aan de knoppen, doe je dat met een zeldzame passie. Soms dwaal je even af naar het opsporen van de ruis maar dat komt door je bijna grenzeloos optimisme. Beste Maurits, ik hoop dat we nog lange tijd op de huidige voet verder kunnen. (Watch the Grappa!)

Dr. Lammers, beste Wim, als op iemand de woorden 'In den beginne...' slaan dan is het wel op joul In blok 1.1 was jij mijn tutor. Dit ontaarde in een revolutionair doch niet als zodanig erkend retrospectief 'griep-onderzoek' waarvan de resultaten nog steeds opzienbarend lijken. Tijdens het schrijven van onze griep-review gaf je mij een kleurendemonstratie van elektrische activatie patronen in het hart. Tijdens het keuzeblok 2.3 was je wederom mijn begeleider en in die periode raakte ik voorgoed verknocht aan 'that good old Ramtek' (we hebben hem onlangs weer operationeel gemaakt!). Beste Wim, ik heb veel van je geleerd en weet zeker dat menig student in Al Ain mij hierin zal volgen.

Mijn beide paranimfen Raymond Smeets en Joseph Brugada.

Beste Raymond, de saaie uren op het studielandschap vlogen voorbij wanneer we in plaats van te studeren debatteerden 'over 'hoe het eigenlijk zou moeten' en 'als wij hier iets te zeggen zouden hebben'. Afsluitende conclusies werden meestal getrokken op weg naar huis of in Brands Bier huis. Achteraf gezien is het misschien maar goed dat fractie ' 83 nooit aan de macht gekomen is. Beste Raymond, het doet me veel plezier jou als paranimf naast mij te hebben bil de verdediging van mijn proefschrift. I $\mathrm{k}$ wens je veel succes toe met je eigen promotie-onderzoek.

Dear Jep, I really hope you'll write your second thesis. Not only because this would be a unique performance by a unique man, but also because this would reflect your daily enthusiasm when studying cardiac circles. Your way of working stimulates everyone. I am honoured to have you as paranimf beside of me and hope that statement number 6 is asked to be defended since this would allow me to promote and announce the 'Brugada-loop". (Stay away from 'Verboden Vruchten').

Prof. R.S. Reneman. Beste Rob, de wijze waarop jij onze vakgroep voorzit en de familie Fysiologie bestuurt geeft niet alleen een veilig gevoel maar is ook een voedingsbodem waarop de wetenschap prima gedilit.

De sfeer op het elektrofysiologisch laboratorium is de basis voor het dagelijks uitoefenen van mijn hobby: prutsen aan het hart. Deze prettige werkomgeving werd 
en wordt nog steeds door velen in stand gehouden. Allereerst door Wieke Rensma en Martin Schalij. Jullie waren mijn 'grote broers' die vele precedenten schiepen waarvan ik nog steeds dankbaar gebruik maak: ik kan ongehinderd een gigantische troep produceren zonder dat iemand er ook maar iets van durft te zeggen, en kan me permiteren om tijdens een minder succesvol experiment met deuren en meubilair te gooien of de haan met gereedschap te bekogelen. Jan Hollen, de eeuwig en altijd klaarstaande alround technicus-electronicus is daadwerkelijk onmisbaar. Beste Jan, ik geef toe dat het wel eens makkelijker is om iets aan jouw te vragen dan het zelf te doen, maar jij weet nu eenmaal alles te regelen. Ik hoop dat je me dit vergeeft (Leve de snoepjesl). De technische ondersteuning van Frits Schmitz, de software-, printer-en PC-vraagbaak functie van Henk Jas, de hulp van Maya Huyberts, Rogier van Drumpt en Maurits Wiffels bil het experimenteren en analyseren van gegevens waren een grote steun en uiterst plezierig. Lucas Boersma die onlangs niet alleen het bier-commissariaat maar ook de Benjamin-rol van mij overnam, wens ik veel succes toe bij zijn promotie onderzoek. Beste Lucas, weldra zal het wispelturige kalium op elliptische wijze bedwongen zijn!

De meeste dank ben ik verschuldigd aan mijn ouders. Jullie waren het die mij een zorgeloze jeugd gaven, mij voortdurend het nut van studie en opleiding deden inzien en dit ook op alle manieren mogelijk maakten. En dan te bedenken hoe ik iedereen ten tijde van 4 Gymnasium te grazen heb genomenl Jullie hielden mij op de juiste momenten de betrekkelijkheid van aardse zaken voor en steunden mij tot op de dag van vandaag in mijn beslissingen, ook al weken die wel eens af van de grootste gemene deler. Hiervoor mijn hartelijke dank.

Tenslotte Juliëtte, mijn allerliefste, toen ik aankondigde te beginnen met het schrijven van dit boekwerk was je al het een en ander gewend en kon je de nabije toekomst wel voorspellen: je zou maandenlang naast je eigen opleidingen en examens ook nog alle andere dagelijkse zaken moeten regelen zodat ik zeven dagen per weak achter mijn PC zou kunnen bivakkeren. Mijn 'schrijf-inspiraties" na 00.00 uur en het daaruit voortvloeiend nachtelijk gecomponeer op het PC-keyboard moeten je toch menigmaal tot wanhoop hebben gedreven. Je liet hier echter niets van merken en bleef mij voortdurend stimuleren terwijl je wist dat mijn tijdsplanningen en 'hetschiet-op' indicaties meestal nergens op sloegen maar enkel een uiting van mijn gemoedstoestand waren. Jouw steun bij het schrijven van dit proefschrift was voor mij onontbeerlijk en als een tweede sinusknoop. Het is ook jouw proefschrift geworden. 


\title{
Curriculum Vitae C.J.H.J. Kirchhof
}

\author{
14-3-1963 geboren te Heerlen \\ 1975-1981 Gymnasium B, Bernardinuscollege te Heerlen \\ 1981 Aanvang studie Geneeskunde Rijksuniversiteit Limburg \\ 1983-1986 Student-assistentschap Fysiologie \\ 1986 Doctoraal examen Geneeskunde \\ 1986-1987 Wetenschappelijk Assistent Fysiologie \\ 1987-heden Universitair Docent Fysiologie
}

